\title{
Computer Aided Multi-scale Design of SiC-Si3N4 Nanoceramic Composites for High-Temperature Structural Applications
}

\author{
Final Technical Report \\ (Jan 01, 2007 to August 31, 2010) \\ Prepared By \\ Vikas Tomar, Ph.D. \\ John E. Renaud, Ph. D., P.E \\ Purdue University, West Lafayette, IN University of Notre Dame, South Dend, IN
}

September 14, 2010

DoE Award Number: DEFG2607NT43072

University of Notre Dame du Lac

511, Main Building

Notre Dame, IN-46556
Purdue University

701 W Stadium Ave

West Lafayette, IN-47907 


\section{DISCLAIMER}

This report was prepared as an account of work sponsored by an agency of the United States Government. Neither the United States Government nor any agency thereof, nor any of their employees, makes any warranty, express or implied, or assumes any legal liability or responsibility for the accuracy, completeness, or usefulness of any information, apparatus, product, or process disclosed, or represents that its use would not infringe privately owned rights. Reference herein to any specific commercial product, process, or service by trade name, trademark, manufacturer, or otherwise does not necessarily constitute or imply its endorsement, recommendation, or favoring by the United States Government or any agency thereof. The views and opinions of authors expressed herein do not necessarily state or reflect those of the United States Government or any agency thereof. 


\section{TABLE OF CONTENTS}

I Executive Summary 4

II Mesoscale Fracture Modeling of $\mathrm{SiC}_{-} \mathrm{Si}_{3} \mathrm{~N}_{4}$ Nanoceramic Composites 5

III Molecular Modeling of Fracture in $\mathrm{SiC}_{-} \mathrm{Si}_{3} \mathrm{~N}_{4}$ Nanocomposites 36

IV A computer Aided Design Methodology to Design Ceramic Nanocomposites 59

V Nanoscale and Mesoscale Creep Experiments 103

VI References $\quad 124$

VII Acknowledgements and List of Publications From Grant Support 134 


\section{EXECUTIVE SUMMARY}

It is estimated that by using better and improved high temperature structural materials, the power generation efficiency of the power plants can be increased by $15 \%$ resulting in significant cost savings. One such promising material system for future high-temperature structural applications in power plants is Silicon Carbide-Silicon Nitride $\left(\mathrm{SiC}_{-} \mathrm{Si}_{3} \mathrm{~N}_{4}\right)$ nanoceramic matrix composites. The described research work focuses on multiscale simulation-based design of these $\mathrm{SiC}-\mathrm{Si}_{3} \mathrm{~N}_{4}$ nanoceramic matrix composites. There were two primary objectives of the research:

1. Development of a multiscale simulation tool and corresponding multiscale analyses of the high-temperature creep and fracture resistance properties of the $\mathrm{SiC}_{-} \mathrm{Si}_{3} \mathrm{~N}_{4}$ nanocomposites at nano-, meso- and continuum length- and timescales; and

2. Development of a simulation-based robust design optimization methodology for application to the multiscale simulations to predict the range of the most suitable phase

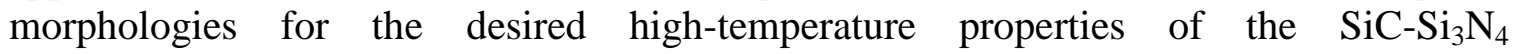
nanocomposites.

The multiscale simulation tool is based on a combination of molecular dynamics (MD), cohesive finite element method (CFEM), and continuum level modeling for characterizing timedependent material deformation behavior. The material simulation tool is incorporated in a variable fidelity model management based design optimization framework.

Material modeling includes development of an experimental verification framework. Using material models based on multiscaling, it was found using molecular simulations that clustering of the $\mathrm{SiC}$ particles near $\mathrm{Si}_{3} \mathrm{~N}_{4}$ grain boundaries leads to significant nanocomposite strengthening and significant rise in fracture resistance. It was found that a control of grain boundary thicknesses by dispersing non-stoichiometric carbide or nitride phases can lead to reduction in strength however significant rise in fracture strength. The temperature dependent strength and microstructural stability was also significantly depended upon the dispersion of new phases at grain boundaries.

The material design framework incorporates high temperature creep and mechanical strength data in order to develop a collaborative multiscale framework of morphology optimization. The work also incorporates a computer aided material design dataset development procedure where a systematic dataset on material properties and morphology correlation could be obtained depending upon a material processing scientist's requirements. Two different aspects covered under this requirement are: (1) performing morphology related analyses at the nanoscale and at the microscale to develop a multiscale material design and analyses capability; (2) linking material behavior analyses with the developed design tool to form a set of material design problems that illustrate the range of material design dataset development that could be performed.

Overall, a software based methodology to design microstructure of particle based ceramic nanocomposites has been developed. This methodology has been shown to predict changes in phase morphologies required for achieving optimal balance of conflicting properties such as minimal creep strain rate and high fracture strength at high temperatures. The methodology incorporates complex material models including atomistic approaches. The methodology will be useful to design materials for high temperature applications including those of interest to DoE while significantly reducing cost of expensive experiments. 


\section{II-Mesoscale Fracture Modeling of $\mathrm{SiC}_{-} \mathrm{Si}_{3} \mathrm{~N}_{4}$ Nanoceramic Composites ${ }^{1}$}

\section{§1. Introduction}

Silicon Carbide ( $\mathrm{SiC})$-Silicon Nitride $\left(\mathrm{Si}_{3} \mathrm{~N}_{4}\right)$ nanocomposites with spherical nano-sized $\mathrm{SiC}$ particles placed either in micro-sized $\mathrm{Si}_{3} \mathrm{~N}_{4}$ grains or along $\mathrm{Si}_{3} \mathrm{~N}_{4}$ grain boundaries (GBs) have been shown to be exceptionally strong at a range of temperatures with maximum temperature upto $1500{ }^{\circ} \mathrm{C}$, [1], [2], and [3]. The first $\mathrm{SiC}_{-} \mathrm{Si}_{3} \mathrm{~N}_{4}$ nanocomposite was prepared by hot-pressing amorphous Si-C-N powders, and these materials contained up to 53 vol\% $\mathrm{SiC}$. Tensile strength data upto $1500 \mathrm{MPa}$, at room temperature, and $970 \mathrm{MPa}$, at $1400{ }^{\circ} \mathrm{C}$ were reported, [4] and [5]. The microstructural morphology of these nanocomposites consists of multiple lengthscales with $\mathrm{Si}_{3} \mathrm{~N}_{4} \mathrm{~GB}$ thickness of the order of $50 \mathrm{~nm}, \mathrm{SiC}$ particle sizes of the order of 200-300 nm, and $\mathrm{Si}_{3} \mathrm{~N}_{4}$ grain size of the order of 0.8-1.5 $\mu \mathrm{m}$, [1], [6], and [7]. The $\mathrm{SiC}$ volume fraction can range from $10 \%$ to as much as $50 \%$, [1]. Depending upon the $\mathrm{SiC}$ phase volume fraction, the grain size as well as the statistical grain size distribution of $\mathrm{Si}_{3} \mathrm{~N}_{4}$ phase can be controlled, [6]. The toughness of the composites depends on the volume fractions of inter- and intragranular SiC dispersions, and controlling these fractions precisely is challenging. Intragranular $\mathrm{SiC}$ particles are thought to be effective in toughening the nanocomposites because they are mainly responsible for crack deflection and crack impediment. However, a clear account of the effect of the $\mathrm{SiC}$ particle placement on the fracture strength of the nanocomposites is not available. A fundamental understanding of the effect of nano-sized $\mathrm{SiC}$ reinforcements on the mechanical behavior of $\mathrm{Si}_{3} \mathrm{~N}_{4}$ matrix composites is required before further attempting to improve the properties of these composites by varied morphological alterations in experiments.

\footnotetext{
${ }^{1}$ A major part of this work has been published in International Journal with Citation "Tomar, V., 2008, Analyses of the role of the second phase $\mathrm{SiC}$ particles in microstructure dependent fracture resistance variation of SiC-Si3N4 nanocomposites, Modelling Simul. Mater. Sci. Eng. 16 (2008) 035001”
} 
The fracture resistance of single phase and multiphase polycrystalline materials has been analyzed in past using analytical, computational, and experimental approaches, [8], [9], [10], [11], [12], and [13]. However, leaving aside some recent promising approaches (such as [14]) analyses have not considered explicit combined role of GBs and second phase particles in the microstructure dependent fracture of these nanocomposites. In the presented research, the cohesive finite element method (CFEM) based dynamic fracture analyses in $\mathrm{SiC}_{-} \mathrm{Si}_{3} \mathrm{~N}_{4}$ nanocomposites with an explicit account of the lengthscales associated with GBs, the second phase $(\mathrm{SiC})$, and the primary phase $\left(\mathrm{Si}_{3} \mathrm{~N}_{4}\right.$ matrix) are performed. For CFEM analyses polycrystalline $\mathrm{SiC}-\mathrm{Si}_{3} \mathrm{~N}_{4}$ nanocomposite structures are generated with grain size of $\mathrm{Si}_{3} \mathrm{~N}_{4}$ approximately equal to $1.2 \mu \mathrm{m}$ (in cases where the GBs are resolved) and the $\mathrm{SiC}$ particle size approximately equal to $200 \mathrm{~nm}$ (Fig. 1). The volume fraction of the $\mathrm{SiC}$ phase is arbitrarily fixed at $20 \%$. The analyses are based on the approach of [15]. The parameters for the interfacial bilinear traction-separation relation are derived using available experimental energy release rate values for $\mathrm{SiC}$ and $\mathrm{Si}_{3} \mathrm{~N}_{4}$. Analyses and discussions focus on delineating the effects of second phase particle placement, loading rate, and phase morphology on the observed dynamic fracture characteristics.

\section{\$2. Formulation}

There are two approaches for analyzing fracture using the CFEM when the crack path is unknown in advance. One is to insert cohesive elements into the model as fracture develops (e.g., [16]). This approach requires the use of specific fracture initiation criteria that are extrinsic to the constitutive model of the material. The other approach is to embed cohesive surfaces along all finite element boundaries as part of the physical model (e.g., [17]). The additional discretization allows the cohesive surfaces to permeate the whole microstructure as an intrinsic part of the 
material characterization. The inclusion of cohesive surfaces along all element boundaries over an area in $2 \mathrm{D}$ or a volume in $3 \mathrm{D}$ allows fracture paths and fracture patterns to be predicted. It assigns a much desired attribute to micromechanical models - the ability to predict fracture pattern and fracture outcome without computational checking at each time step as to which branch of material model to follow in a specific material location. By using this approach it may be possible that the elastic stiffness of material gets reduced in the case of cohesive laws having finite initial slope. However, a convergence criterion developed and reported in [18] eliminates such problems. We follow the second approach based on the work of [18] in our analyses. Each phase is modeled with hyperelastic constitutive behavior based on available experimental evidence, [19], [20], [21], and [22]. In the absence of the experimental information, the distribution of the crystalline orientation of the $\mathrm{Si}_{3} \mathrm{~N}_{4}$ and $\mathrm{SiC}$ phases is neglected.

\section{\$2.1 CFEM framework}

A Lagrangian finite deformation formulation is used to account for the finite strains and rotations in crack tip regions. The transient finite deformation kinetics is specified through the principle of virtual work as

$$
\int_{V} \mathbf{s}: \delta \mathbf{F} \mathrm{d} V-\int_{S_{i n t}} \mathbf{T} \cdot \delta \boldsymbol{\Delta} \mathrm{d} S=\int_{S_{\text {ext }}} \mathbf{T} \cdot \delta \mathbf{u} \mathrm{d} S-\int_{V} \rho \frac{\partial^{2} \mathbf{u}}{\partial t^{2}} \cdot \delta \mathbf{u} \mathrm{d} V
$$

where $\mathbf{s}: \delta \mathbf{F}=\mathrm{s}^{i j} \delta \mathrm{F}_{j i}$ in Cartesian coordinates; $\mathbf{s}$ is the first Piola-Kirchoff stress; $\boldsymbol{\Delta}$ is the displacement jump across a pair of cohesive surfaces; $V, S_{\text {ext }}$ and $S_{\text {int }}$ are the volume, external surface area and internal cohesive surface area, respectively, of the body in the reference configuration. The density of the material in the reference configuration is $\rho . \delta \mathbf{F}, \delta \boldsymbol{\Delta}$, and $\delta \mathbf{u}$ denote admissible variations in $\mathbf{F}, \Delta$ and $\mathbf{u}$, respectively. The traction vector $\mathbf{T}$ and the surface normal $\mathbf{n}$ in the reference configuration are related through $\mathbf{T}=\mathbf{n} \cdot \mathbf{s}$. In the continuum 
interfacial separation relations, the traction $\mathbf{T}$ applied on material points $P$ and $P^{\prime}$ coinciding at and occupying position $\mathbf{x}$ on cohesive surface $S_{0}$ in the reference configuration is workconjugate to surface separation $\Delta$. Reckoned in the reference configuration, the cohesive law is

$$
\mathbf{T}(\mathbf{x})=\mathbf{T}[\Delta(\mathbf{x})]
$$

and the work of separation under this traction at any stage of deformation is ([23]),

$$
\mathrm{W}_{\text {sep }}=\int_{S_{0}}^{\Delta} \int_{0}^{\Delta} \mathbf{T}(\mathbf{x}) \cdot \mathrm{d} \Delta \mathrm{d} S
$$

Implied here is the assumption that cohesive traction-separation relations are locally determined, i.e. the cohesive traction at one point is fully determined by the separation at the point itself. A review of various types of cohesive laws is given by, e.g., [24]. We use the CFEM framework described in [18].

\section{\$2.1.1 Irrversible bilinear cohesive law}

The CFEM simulations are carried out under plane strain assumption. Although the discussion in the presented research focuses on tensile loading, compression and contact can be dealt with as well within this framework, [25]. The irreversible bilinear law used can be regarded as a generalized version of the cohesive laws with irreversibility, [26] and [27]. Similar to these laws,

we have an internal state variable $\lambda$ defined as $\lambda=\sqrt{\left(\Delta_{n} / \Delta_{n c}\right)^{2}+\left(\Delta_{t} / \Delta_{t c}\right)^{2}}$ that describes the effective instantaneous state of mixed-mode separations. The traction and the cohesive surface separation are related through a description $\Phi$ of the surface energy dissipation per unit area which is a function of separation vector $\Delta$ through the state variable $\lambda$. Here, $\Delta_{n}=\mathbf{n} \cdot \Delta$ and $\Delta_{t}=\mathbf{t} \cdot \boldsymbol{\Delta}$ denote, respectively, the normal and tangential components of $\Delta$, with $\mathbf{n}$ and $\mathbf{t}$ being unit vectors normal and tangent to initial cohesive surface $S_{0}$, respectively. $\Delta_{n c}$ is the critical 
normal separation at which the cohesive strength of an interface vanishes under conditions of pure normal deformation $\left(\Delta_{t}=0\right)$. Similarly, $\Delta_{t c}$ is the critical tangential separation at which the cohesive strength of an interface vanishes under conditions of pure shear deformation $\left(\Delta_{n}=0\right)$. In addition to $\lambda$ a parameter $\eta=\max \left\{\eta_{0}, \lambda_{u l}\right\}$ is defined to account for the irreversibility of separations. As illustrated in Fig. 2(a), $\eta_{0}$ is the initial value of $\eta$ which defines the stiffness of the original undamaged cohesive surface and $\lambda_{u l}$ is the hitherto maximum value of $\lambda$ at which an unloading process was initiated. While $\eta_{0}$ is the characteristic value of effective separation $\lambda$ at which the effective traction $\sigma$ (see below) for a cohesive surface pair reaches the strength $\mathrm{T}_{\max }$ of the undamaged surface, $\lambda_{u l}$ is the critical level of $\lambda$ at which $\sigma$ reaches the reduced strength $\mathrm{T}_{\max }(1-\eta) /\left(1-\eta_{0}\right)$ of the hitherto damaged cohesive surface pair. The specific form for $\Phi$ is taken as

$$
\Phi=\Phi(\lambda, \eta)= \begin{cases}\Phi_{0}\left(\frac{1-\eta}{1-\eta_{0}}\right)\left(\frac{\lambda^{2}}{\eta}\right), & \text { if } 0 \leq \lambda \leq \eta \\ \Phi_{0}\left(\frac{1-\eta}{1-\eta_{0}}\right)\left(1-\frac{(1-\lambda)^{2}}{1-\eta}\right), & \text { if } \eta<\lambda \leq 1 .\end{cases}
$$

This relation allows the traction to be defined through

$$
\mathbf{T}=\frac{\partial \Phi}{\partial \boldsymbol{\Delta}}
$$

yielding the normal and shear traction components as,

$$
\mathrm{T}_{n}=\sigma(\lambda, \eta) \frac{\Delta_{n}}{\lambda \Delta_{n c}} \quad \text { and } \quad \mathrm{T}_{t}=\sigma(\lambda, \eta) \frac{\alpha \Delta_{t}}{\lambda \Delta_{t c}}
$$

In the above expressions, $\alpha=\Delta_{n c} / \Delta_{t c}$ and 


$$
\sigma=\sqrt{\left(\mathrm{T}_{n}\right)^{2}+\left(\mathrm{T}_{t} / \alpha\right)^{2}}= \begin{cases}\left(\mathrm{T}_{\max } \frac{1-\eta}{1-\eta_{0}}\right) \frac{\lambda}{\eta}, & \text { if } 0 \leq \lambda \leq \eta \\ \left(\mathrm{T}_{\max } \frac{1-\eta}{1-\eta_{0}}\right) \frac{1-\lambda}{1-\eta}, & \text { if } \eta<\lambda \leq 1 ; \\ 0, & \text { if } \lambda>1 .\end{cases}
$$

The work of separation for forming a fractured surface per unit surface area is

$$
\int_{0}^{\Delta_{c}} \mathbf{T} \cdot \mathrm{d} \Delta=\frac{1}{2} \mathrm{~T}_{n}^{\max } \Delta_{n c}=\frac{1}{2} \alpha \mathrm{T}_{n}^{\max } \Delta_{t c}=\Phi\left(1, \eta_{0}\right)=\Phi_{0}
$$

While the bilinear relationship between $\sigma$ and $\lambda$ embodied in the above formulation is illustrated in Fig. 2(a), the variation of $\Phi$ is shown in Fig. 2(b). Based on Eq. (7) the amount of work that has been dissipated, $\Phi_{d}$, is

$$
\Phi_{d}(\lambda, \eta)=\left\{\begin{array}{l}
0, \text { if } \lambda \leq \eta_{0} \\
\Phi\left(\eta, \eta_{0}\right)-\Phi(\eta, \eta)=\frac{\eta-\eta_{0}}{1-\eta_{0}} \Phi_{0}, \text { if } \eta_{0}<\lambda \leq \eta \\
\Phi\left(\lambda, \eta_{0}\right)-\Phi(\lambda, \eta)=\frac{\lambda-\eta_{0}}{1-\eta_{0}} \Phi_{0}, \text { if } \eta<\lambda \leq 1 ; \\
\Phi_{0}, \text { if } \lambda>1 ;
\end{array}\right.
$$

When full separation is achieved, $\Phi_{d}(1, \eta)=\Phi_{0} . \Phi_{d}$ is partly converted into the surface energy and partly spent on causing damage in the material adjacent to crack surfaces through microcrack formation that is not based on a pre-specified criterion. A unique damage parameter can be defined to phenomenologicaly track the progressive softening of cohesive surfaces interspersed throughout the composite microstructure. This parameter D is defined such that

$$
\mathrm{D}=\frac{\Phi_{d}}{\Phi_{0}}
$$

Note that $0 \leq D \leq 1$, with $D=0$ indicating fully recoverable interfacial separation and $D=1$ signifying complete separation or total fracture. In the numerical analysis carried out in [15], [28], and in the presented research D is used as a state variable quantifying the degree of the damage, 
providing a phenomenological measure for failure analysis. The spatial and time variation of $\mathrm{D}=\mathrm{D}(\mathbf{x}, t)$ allows the distribution and evolution of damage in various microstructures to be analyzed.

\section{\$2.1.2 Experiment based calculation of the bilinear cohesive law parameters}

Overall, five parameters are needed to specify the cohesive behavior, including the maximum tensile strength $\mathrm{T}_{\max }$, the critical separations $\Delta_{n c}$ and $\Delta_{t c}$, characteristic separation $\eta_{0}$, and $\alpha$. Note that only four of these parameters are independent since $\alpha=\Delta_{n c} / \Delta_{t c}$. The calibration of these parameters is an important aspect in the implementation of the CFEM model. $\mathrm{T}_{\max }$ is commonly assumed to be a fraction of the Young's modulus (1/100 to 1/1000), [17]. The critical separations $\Delta_{n c}$ and $\Delta_{t c}$ are usually obtained by equating the area under the cohesive relation to the formation energy per unit area of the corresponding fracture surface. In this regard, experimental efforts have been reported, [29] and [30]. The value of $\alpha$ is typically obtained from the ratio between the tangential and normal energy release rates, [31]. In this paper, the approach for parameter selection as described by [17] is used. The value of characteristic separation $\eta_{0}$ is taken as $0.001,[30]$. GBs in the nanocomposites have glassy structure consisting of densification aids such as $\mathrm{Y}_{2} \mathrm{O}_{3}$ and other rare earth oxides such as samarium, gadolinium, dysprosium, erbium, and yetterbium (verified using the TEM observations on the $\mathrm{Si}_{3} \mathrm{~N}_{4}$ phase, [32]). Experimental data on the fracture properties and strength of GBs is not available. The GB's chemical composition is a complex and uncharacterized combination of different compounds such as $\mathrm{Y}_{2} \mathrm{O}_{3}, \mathrm{MgO}$ etc. with glassy structure. Accordingly, the fracture properties for GBs cannot be specified based on chemical composition. However, experiments for polycrystalline $\mathrm{Si}_{3} \mathrm{~N}_{4}$ have shown that the presence of GBs results in lowering of mechanical strength owing to GB sliding (attributed to glassy structure) and increase in fracture strength owing to the crack- 
deflection effect (roughly of the order of $5 \%$ ), [2]. Accordingly, the GBs are arbitrarily assigned $5 \%$ higher fracture strength than that of the $\mathrm{Si}_{3} \mathrm{~N}_{4}$ matrix. Increase in fracture strength for structural and glassy materials is often accompanied with reduction in elastic modulus. Accordingly, while arbitrary assigning 5\% higher fracture toughness to GBs than $\mathrm{Si}_{3} \mathrm{~N}_{4}$ phase, the elastic modulus is made smaller by $5 \%$ than that of the $\mathrm{Si}_{3} \mathrm{~N}_{4}$ phase. The immediate effect is that the simulations are qualitative in nature. With availability of experimental measurements on GB properties, more realistic properties of GBs can be incorporated to increase the accuracy of the simulation predictions.

Because the GBs have finite widths, there are three phases (GBs, $\mathrm{SiC}$, and $\mathrm{Si}_{3} \mathrm{~N}_{4}$ ) in the microstructures analyzed. The cohesive parameters are calculated using the experimental information on the elastic moduli and $\Phi_{0},[6],[20]$, [33], [34], [35], [36], [19],[21], [22]. Homogenized properties are calculated using volume weighted averaging. The values for $\Phi_{0}$ are obtained based on surface energy release rate measurements during fracture experiments on bulk $\mathrm{SiC}$ and $\mathrm{Si}_{3} \mathrm{~N}_{4}$ reported in the literature. An interface between any two of the three phases is assigned the cohesive properties corresponding to the weaker phase. Table 1 shows the material properties for analyzing microstructures shown in Fig. 1.

\section{\$2.1.3 Finite element discretization}

A lumped mass matrix is used in the finite element discretization. Explicit time-integration scheme based on the Newmark $\beta$-method with $\beta=0$ and $\gamma=0.5$ is employed, [37]. The magnitude of time increment $\Delta t$ in the explicit integration is based on the Courant-FreidrichsLewy criterion and material-related numerical stability considerations for explicit time integration. The time step $\Delta t$ also needs to be small enough to ensure that the increment of the cohesive separation $\Delta$ (or $\Delta \lambda$ ) be sufficiently small in each numerical step to avoid numerical 
instability. An empirical criterion of $\Delta \lambda<0.1 \eta$ is used, [38]. Based on the material properties (see Table 1) and the Courant-Freidrichs-Lewy criterion with material-related numerical stability considerations the average timestep is of the order of $0.6 \mathrm{ps}$ which is approximately one tenth of the time it takes for the longitudinal wave to traverse the smallest bulk element in the meshes in current research.

\section{\$2.2 Problem setup}

Microstructures analyzed in the current research are shown in Fig. 1. Since, a given unique set of phase morphology defining parameters (such as the location of SiC particles in the current research) corresponds to multiple sets of phase morphologies, three different random samples corresponding to each microstructural representation shown in Fig. 1 (Class-I, Class-II, and Class-III) are used to characterize the material behavior. Microstructures have grain size of $\mathrm{Si}_{3} \mathrm{~N}_{4}$ approximately equal to $1.2 \mu \mathrm{m}$ and the $\mathrm{SiC}$ particle size approximately equal to $200 \mathrm{~nm}$. The volume fraction of the SiC phase is fixed at $20 \%$. The average GB width is approximately $120 \mathrm{~nm}$. Accordingly, the microstructures with GB have the approximate GB volume fraction of $10 \%$.

To track complex crack/microcrack patterns, arbitrary crack paths, and crack branching, cohesive surfaces are specified along all finite element boundaries as an intrinsic part of the finite element model. All cohesive surfaces serve as potential crack paths in the microstructure; therefore, fracture inside each microstructural phase and along interphase boundaries can be explicitly resolved, (for more details see [18], [28], and [15]). Accordingly, the analyses are able to take into account the intergranular as well as intragranular fracture. The finite element meshes used have a uniform structure with "cross-triangle" elements of equal dimensions arranged in a quadrilateral pattern, Fig. 3. This type of triangulation is used since it gives the maximum 
flexibility for resolving crack extensions and arbitrary fracture patterns, [18]. Because of the computational limitations and the requirement that stress wave reflections do not interfere with the analyses results, [18], the microstructures are embedded in a uniform finite element mesh, (see the mesh surrounding the microstructure in Fig. 3). The uniform mesh has elements with higher size increasing the overall size of the sample to delay the stress wave reflection and minimize its effect on dynamic fracture while simultaneously reducing the computational load. Since the crack propagation is limited to the microstructural window whose size has been analyzed in a previous research, [18], results are unaffected by the presence of the uniform mesh.

The dimensions for the microstructural region $(7.5 \mu \mathrm{m} \times 30 \mu \mathrm{m})$ are limited by the memory sizes of the super-computers (a 48 processor Opteron Linux cluster) in this work. These regions are much larger than the length scales involved in all microstructures. Thus, reasonable representations of the microstructures are achieved. Material outside the microstructure window is assumed to be homogeneous and assigned effective properties representative of those for the $\mathrm{SiC}_{-} \mathrm{Si}_{3} \mathrm{~N}_{4}$ ceramic composite. Computations are carried out for side-cracked samples under tensile loading. The length of the initial crack is $a_{\mathrm{i}}=9.0 \mu \mathrm{m}$. Tensile loading is applied by imposing velocity boundary conditions along the upper and the lower edges of the specimen in the direction shown in Fig. 3. The boundary velocity $V_{o}(0.5 \mathrm{~m} / \mathrm{sec}$ and $2 \mathrm{~m} / \mathrm{sec})$ is imposed on the bottom and top edges with a linear ramp from zero to $\mathrm{V}_{\mathrm{o}}$ in the initial phase of loading. This represents the loading of the pre-crack by a tensile wave with a stress amplitude of $\sigma=\rho \mathrm{C}_{\mathrm{L}} \mathrm{V}_{0}(\rho$ is homogeneous material density and $\mathrm{C}_{\mathrm{L}}$ is the homogeneous material longitudinal wave velocity) (14.3 MPa and 57.3 MPa at $\mathrm{V}_{\mathrm{o}}=0.5 \mathrm{~m} / \mathrm{sec}$ and $2 \mathrm{~m} / \mathrm{sec}$, respectively) and a linear ramp from zero to that value in the initial phase of loading. The specimen is stress free and at rest initially. Conditions of plain strain are assumed to prevail. 
For the CFEM mesh and the bilinear cohesive law used in the current research, the convergence analyses of the dependence of mesh element size on simulation results have already been performed, [18]. Based on those analyses, we require the characteristic finite element size, $h$, to satisfy: $750 \mathrm{~nm}>h \geq 30 \mathrm{~nm}$. The upper limit is based on the minimum cohesive zone size estimate based on the properties listed in Table 1, [18]. The lower limit is based on the elimination of material softening because of the use of bi-linear law with finite initial stiffness, [18]. The characteristic element size of $75 \mathrm{~nm}$ used in the current research satisfies the convergence criterion. The characteristic size corresponds to 4 finite elements, Fig. 3. Accordingly, there are 542000 elements in each analyzed FE mesh.

\section{§3 Dynamic Fracture Analyses}

Simulations solely focus on analyzing the role of $\mathrm{SiC}$ particles and GBs in the dynamic fracture of $\mathrm{SiC}_{-} \mathrm{Si}_{3} \mathrm{~N}_{4}$ nanocomposites. Focus is also on analyzing the role of the loading rate variation. Results are analyzed both in terms of the dependence of fracture energy dissipation on the nanocomposite microstructural features as well as in terms of the changes in damage evolution and stress distribution in the nanocomposites associated with changes in the phase morphology.

\section{\$3.1 Stress distribution and damage evolution in the microstructures}

In order to understand the stress distribution and damage evolution, the damage parameter (D-Eq. (10)), and the maximum principal stress $\left(\sigma_{\max }\right)$ distributions are analyzed in all microstructures. As explained earlier $0 \leq \mathrm{D} \leq 1$, with $\mathrm{D}=0$ indicating fully recoverable interfacial separation and $\mathrm{D}=1$ signifying complete separation or fracture. The results for changes in stress distribution as a function of time in all three types of microstructures (Fig. 4) and for damage 
distribution in all three types of microstructures at time $t=0.01875 \mu \mathrm{sec}$ (Fig. 5) at $\mathrm{V}_{\mathrm{o}}=2 \mathrm{~m} / \mathrm{sec}$ are discussed. The results at velocity $\mathrm{V}_{\mathrm{o}}=0.5 \mathrm{~m} / \mathrm{sec}$ are similar and, therefore, are not discussed.

In Fig. 4 , at time $t=0.01875 \mu \mathrm{sec}$ a concentrated stress field similar to homogeneous materials near the propagating crack-tip is seen in all three microstructures. The distribution of stress around crack tip is strongly affected by the distribution of second phase $\mathrm{SiC}$ particles w.r.t. $\mathrm{Si}_{3} \mathrm{~N}_{4}$ GBs. Presence of $\mathrm{SiC}$ particles inside $\mathrm{Si}_{3} \mathrm{~N}_{4}$ grain interior in the case of Class-I and Class-III microstructures results in stress concentration over and in excess to the crack-tip stresses that are seen in the case of Class-II microstructure where the SiC particles are placed solely along GBs. In the case of Class-II microstructure near crack-tip stresses are concentrated in a very small region. This is contrary to the case of Class-I microstructure where the near crack-tip stress fields are spread over larger microstructural region. Owing to the SiC particle stress concentration, intergranular micro-cracks originating in the microstructure can also be seen. Stress distribution in the Class-III microstructure lies in between those for Class-I and Class-II microstructures. The effect of the difference in the fracture and bulk properties of GBs and $\mathrm{Si}_{3} \mathrm{~N}_{4}$ matrix on stress distribution is insignificant in comparison to the effect of the second phase particle placement. GBs are shown to restrict the damage to grain interior. However, SiC particles primarily determine how soon the structure develops microcracks. In Fig. 4, at time $t=0.02275 \mu$ sec the stress distribution after the microstructures are subjected further to $0.1 \%$ strain is shown. While the cracking is significant in Class-I and Class-III microstructures, the Class-II microstructure is still intact. Predominant mode of catastrophic fracture initiation in all microstructures is microcrack formation in $\mathrm{Si}_{3} \mathrm{~N}_{4}$ grains interiors. After the initial micro-crack formation, the load is also shared by GBs and second phase particles which ultimately results in brittle fragmentation of the microstructures. As expected, Class-II has the highest fracture initiation resistance. 
The contours of the D distributions in Fig. 5 show similar topological characteristic to that of the $\sigma_{\max }$ in Fig. 4 at time $t=0.01875 \mu \mathrm{sec}$. There is a clear correlation between the stress distribution in Fig. 4 at time $t=0.01875 \mu \mathrm{sec}$ and the microcrack density in all microstructures shown in Fig. 5. Stress is the maximum at points where D changes from zero to a non-zero value and is close to zero corresponding to the points with $\mathrm{D}=1$. The distribution as well as the propagation of damage is influenced by the nature of distribution of the second phase particles. Damage is the maximum in the stress concentration regions corresponding to $\mathrm{SiC}$ particles. Damage initiation is primarily intergranular in nature. However, some amount of intra-granular cracking is seen in the case is the $\mathrm{SiC}$ particles are present along GBs. In order to separate the effect of GBs from that of the second phase particles on the fracture strength and to demonstrate the strong influence of the GB properties on the fracture resistance, a comparison of damage progression in a Class-I microstructure in both the presence and absence of GBs is shown in Fig. 6. As shown, highly fracture resistant GBs limit the damage to a much smaller near crack tip region in turn limiting the rate of damage progression and making the microstructure stronger.

A comparison of damage and stress distributions in all microstructures reveals that the second phase particles placed in the interior of the grains have stronger effect on causing fracture initiation in comparison to the ones placed along GBs. The second phase particles in the $\mathrm{Si}_{3} \mathrm{~N}_{4}$ grain interior cause stress concentration that is resisted by the soft crack resisting GBs when the particles are present along GBs. This is not the case when the particles are present only in the grain interior. Damage initiation to the microcrack formation occurs fastest in the Class-I microstructure indicating the least fracture initiation resistance. The contours of the parameter D indicate that dissipation through microcracking occurs away from the main cracks. Apart from the main crack, microcracks form along whole microstructure. Owing to the inhomogeneous 
microstructure, most of the microcracks do not have enough area available to propagate over a long distance. Accordingly, the crack formation needs to be characterized in terms of the normalized crack density, $a$, (sum of the length of all cohesive surfaces with $\mathrm{D} \geq 1$ normalized with the sum of the length of all cohesive surfaces) than in terms of crack length of the main crack.

\section{\$3.2 Normalized crack density evolution and cohesive energy dissipation}

The time histories of the mean values of normalized crack density as a function of loading time at both loading rates are shown in Fig. 7. Figure 8 shows the overall cohesive energy dissipation in the microstructures as a function of normalized crack density values. The overall cohesive energy dissipated $\phi_{d}$ is the sum of cumulative energy dissipated in all the damaged

cohesive surfaces, i.e. $\phi_{d}=\int_{S_{d}} \Phi_{d} \mathrm{~d} S$ (Eq. (9)), where $S_{d}$ represents surface area of cohesive surfaces with damage ( $\mathrm{D}>0$ ). The energy dissipated is due to the crack surface separation and microcrack nucleation. It is one measurement of the fracture resistance of the samples under the conditions analyzed. From the plots of $\phi_{d}$ as a function of the normalized crack density, $a$, we can readily obtain the average energy release rate $\left(\overline{\mathrm{G}}=\phi_{d} / a\right)$ which provides a quantitative measure of the dynamic fracture resistance of materials. The curves in the Figs. 7 and 8 correspond to the mean values of the plotted quantity measured from three different random samples corresponding to each type of microstructure (Class-I, Class-II, and Class-III). Error bars in the Figs. 7 and 8 correspond to the variations in the plotted values corresponding to three different samples analyzed for each type of microstructure. In the following discussions we analyze results in Figs. 7 and 8, simultaneously.

It is important to note that the initial normalized crack density value in Fig. 7 is zero. Therefore, the plotted normalized crack density here concerns the normalized value of the length 
of new crack surfaces w.r.t. total available microstructural surface length for crack formation but not the initial crack. As established earlier, owing to relatively higher fracture strength of the GBs, the initial crack mainly propagates in the matrix resulting in intragranular fracture initiation in all microstructures at both loading rates. At both loading rates, the presence of the second phase particle along GBs in Class-II microstructure increases the fracture initiation time. The second phase particles in the $\mathrm{Si}_{3} \mathrm{~N}_{4}$ grain interior cause stress concentration that is resisted by the soft crack resisting GBs when the particles are present along GBs. This is not the case when the particles are present only in the grain interior. Once initiated, cracks propagate rapidly to failure in each microstructure irrespective of the magnitude of loading rate and the crack initiation time. At $2 \mathrm{~m} / \mathrm{sec}$ loading rate, the mean crack growth rate is the same for Class-I and Class-III microstructures. At $0.5 \mathrm{~m} / \mathrm{sec}$ the mean crack growth rate is the highest in the Class-III microstructure. The presence of SiC particles in the Class-I microstructure primarily causes the intergranular microcrack initiation. In Class-III microstructure, however, the stress concentrating $\mathrm{SiC}$ particles are also present along the GBs. This suggests that $\mathrm{SiC}$ particles in Class-III microstructures may be responsible for intergranular microcrack initiation as well as crack propagation along the GB-microstructure interface. One unexpected result is that the variation in the normalized crack density value for Class-III microstructures is the highest at both loading rates. Damage propagation analyses revealed that the crack bridging effect of the $\mathrm{SiC}$ particles is responsible for such large variation. If the particles are in the wake of the micro-cracks, the crack bridging tends to lower the crack growth rate. However, if the particles are in the front of the micro-crack, the resulting stress concentration would tend to accelerate the crack growth. If the $\mathrm{SiC}$ particles are lying close to GBs, they invariably reduce the crack growth rate by causing the crack bridging. However, if the particles are concentrated in grain interior away from the GBs 
and also are present along GBs then the resulting microstructure is the weakest. Largest variation for the Class-III microstructure results from these effects. As shown in Fig. 8, at both loading velocities the mean values of energy dissipation is nearly the same for all three types of microstructures. Equal mean values of the energy dissipation for Class-II and Class-III microstructures to the Class-I microstructure imply that the primary mechanism of fracture is intergranular $\mathrm{Si}_{3} \mathrm{~N}_{4}$ microcrack initiation (i.e. primarily only one phase is getting fragmented) and propagation in the bulk or along the matrix-GB interfaces. Based on the mechanism explained in the previous paragraph, the energy dissipation for the Class-III microstructure has the highest variability.

Combination of energy and crack density plots shows that Class-II is the strongest microstructure against crack initiation since it is dissipating more energy for the same amount of crack length change thereby increasing the energy release rate, $\overline{\mathrm{G}}$. However, high levels of variations in the crack density and energy dissipation values for the Class-III microstructure imply that a judicious placement of second phase particles along GBs and near GBs in grain interior may result in the Class-III microstructure being the strongest of the microstructures. In order to further analyze this issue, the cohesive energy dissipation and its variation corresponding to the normalized crack density values of 0.1 and 0.15 at both loading rates were used to calculate the average energy release rate, $\overline{\mathrm{G}}_{\text {ave }}\left(\left(\phi_{\text {ave }}^{0.15}-\phi_{\text {ave }}^{0.10}\right) /\left(a_{\text {ave }}^{0.15}-a_{\text {ave }}^{0.10}\right)\right.$, "ave" stands for average) and maximum energy release rate $\overline{\mathrm{G}}_{\max }\left(\left(\phi_{\max }^{0.15}-\phi_{\min }^{0.10}\right) /\left(a_{a v e}^{0.15}-a_{a v e}^{0.10}\right)\right.$, "max" stands for maximum and min stands for minimum). $\overline{\mathrm{G}}_{\text {ave }}$ is shown in Fig. 9 (a) and $\overline{\mathrm{G}}_{\max }$ is shown in Fig. 9 (b). It is clear from Fig. 9 (a) at both loading rates that mean propagation fracture toughness (fracture toughness as a function of increasing crack density) is the highest in the case of Class-II. However, by accounting for the variations, it becomes clear that Class-III 
microstructure with the judicious placement of the second phase particles along the GBs and grain interiors in the wake of the cracks is the strongest microstructure, Fig. 9(b).

Loading rate has a clear impact on dynamic behavior of materials, primarily due to the inertial effect. An analysis of the plotted results shows the dependence of the mean and the variations of the plotted quantities on the loading rate. In particular, the crack propagation fracture toughness increases with the loading rate, Fig. 9 (a). The variations, however, are higher for smaller loading rate (Fig. 9 (b)) indicating that the phase morphology has stronger role to play at smaller loading rates. At the higher loading rate of $2 \mathrm{~m} / \mathrm{sec}$, the effect of the inertia of the microcracks supersedes the phase morphology effect. Experiments have revealed many characteristics of dynamic fracture under different loading rates, [39]. Multiple length scales in a microstructure give rise to more complicated interactions under dynamic loading. Crack tip instabilities including deflection and branching, can arise form the combined influence of microstructure and higher loading rates. As shown in Fig. 9, average energy release rate increases with increasing loading rate, indicating more extensive damage at higher stress levels arising from higher loading rates.

\section{§4 Discussions and Validations}

Self-reinforced $\mathrm{Si}_{3} \mathrm{~N}_{4}$ is an important example of the high-toughness structural ceramics first manufactured during 1970s. Embedding nanosized $\mathrm{SiC}$ particles in the $\mathrm{Si}_{3} \mathrm{~N}_{4}$ matrix is one proven approach to improve its mechanical properties. It is also verified in the current research by comparing the average energy release rate of all the microstructures with that of a control microstructure that contains no-SiC particle at all, Fig. 10. As shown, a significant increase in the fracture resistance is observed. For the first time, [3] reported almost $200 \%$ improvements in both the fracture toughness and the strength of $\mathrm{SiC}_{-} \mathrm{Si}_{3} \mathrm{~N}_{4}$ nanocomposites by embedding nanometer size $(20-300 \mathrm{~nm})$ particles within a matrix of larger grains and at the $\mathrm{Si}_{3} \mathrm{~N}_{4} \mathrm{GBs}$. 
Since then, a number of experiments focusing on manufacturing $\mathrm{SiC}_{-} \mathrm{Si}_{3} \mathrm{~N}_{4}$ nanocomposites have been reported, [1] and [2]. So far it has been very difficult to control the processing routes and conditions to place $\mathrm{SiC}$ particles selectively along GBs. However, it is possible to place second phase particles in a combination that places them along GBs as well as in grain interiors near GBs which may ultimately resulted in very high strength $\mathrm{SiC}_{-} \mathrm{Si}_{3} \mathrm{~N}_{4}$ nanocomposites, [40]. Such mechanisms have been observed earlier for other SiC particle reinforced nanocomposites, [4] and [41].

From Figs. 4, 5, and 6 one may argue that the formation of a large damage zone around the main crack implies that the material is weaker at the microscale. However, such material is also dissipating fracture energy that would otherwise contribute to advancing the main crack. Since it is the main crack that ultimately separates the material (at the macro-scale) and controls tensile strength, anything that can impede its advance, including the formation of microcracks, is beneficial. Indeed such reasoning is an important alternative to the explained failure mechanism. However, at the size scale of analyses the ultimate failure in all microstructures occurred by the propagation of diffusive microcracks as shown in Fig. 5. Future research that integrates analyses at such small size scale with higher length-scale analyses (of the order of millimeters) to understand the final failure mechanism should be performed to answer this question. At the current length-scale of problem and the corresponding computational limitations this questions cannot be answered.

The current simulation setup solely focuses on dynamic fracture. Accordingly, a direct comparison of the calculated average energy release rate values with the experimentally available fracture toughness values is not possible. However, the order of fracture toughness values calculated based on the observed average $\overline{\mathrm{G}}$ values and homogenized Young's moduli 
values are close to the values reported for the nanocomposites in the literature. In addition, the computationally observed fracture mechanisms are in accordance with experimental observations on similar other material systems. The dissipated cohesive energy values as a function of normalized crack density values intricately depend upon the type of microstructure morphology, the applied loading rate, and the volume fractions of $\mathrm{GBs}$ and $\mathrm{SiC}$ particles. The reported analyses are for only one set of values of the GB and SiC particle volume fraction. In order to form a mathematical description, additional runs at a range of volume fraction values are required, which is the subject of ongoing research.

\section{$\S 5$ Conclusions}

A CFEM framework for analyzing fracture in polycrystalline $\mathrm{SiC}-\mathrm{Si}_{3} \mathrm{~N}_{4}$ nanocomposites is described. Analyses are carried out on microstructures with $\mathrm{SiC}$ and $\mathrm{Si}_{3} \mathrm{~N}_{4}$ having two different fixed grain sizes with GBs resolved using the characteristic finite element size of $75 \mathrm{~nm}$. Calculations are carried out on a range of microstructures with purely intergranular dispersion of the second phase $\mathrm{SiC}$ particles, microstructures with purely intragranular dispersion of $\mathrm{SiC}$ particles, and microstructures with a mixed combination (along GBs and inside grains) of $\mathrm{SiC}$ particle dispersion. Analyses demonstrate that;

1. Irrespective of the location of second phase particles the final failure mode in all microstructures is found to be brittle fragmentation with initial micro-cracks forming and propagating mainly in $\mathrm{Si}_{3} \mathrm{~N}_{4}$ matrix. Later part of brittle fragmentation includes $\mathrm{GB}$ and $\mathrm{SiC}$ fragmentation;

2. Second phase particles have two important effects. If they are present in front of the crack tip they weaken the microstructure because of stress concentration cause by them. However, if 
they are present near GBs they cause the crack bridging effect with increase in the strength of the microstructure;

3. Class-II microstructures on an average are the strongest. However, by careful dispersion of second phase particles near GBs and along GBs the Class-III microstructures can be made the strongest.; and

4. Class-III microstructures represent the best combination of fracture resistance and mechanical strength. This is important in light of experimental development of these advanced materials where the control of processing to produce exclusively Class-II morphology is a very difficult task. 
TABLE 1. Bilinear Cohesive Law Parameters for Different Phases in Microstructures (Phase volume fractions $\left.\left(V_{f}\right)_{\mathrm{SiC}}=0.2\left(V_{f}\right)_{\mathrm{Si} 3 \mathrm{~N} 4}=0.7\left(V_{f}\right)_{\mathrm{GB}}=0.1\right)$

\begin{tabular}{|c|c|c|c|c|c|c|}
\hline Component & $\boldsymbol{\Phi}_{\mathbf{0}}(\mathrm{N} / \mathrm{m})$ & $\mathbf{T}^{\mathbf{m a x}}(\mathrm{GPa})$ & $\Delta(\mathrm{nm})$ & $\boldsymbol{E}(\mathrm{GPa})$ & $\boldsymbol{v}$ & $\boldsymbol{\rho}\left(\mathrm{kg} / \mathrm{m}^{3}\right)$ \\
\hline $\mathrm{SiC}_{(\mathrm{sc})}$ & 19.53 & 1.02 & 38.3 & 449 & 0.16 & 3215 \\
\hline $\mathrm{Si}_{3} \mathrm{~N}_{4}(\mathrm{sn})$ & 191.5 & 2.3 & 166.5 & 210 & 0.22 & 2770 \\
\hline $\mathrm{GB}(\mathrm{g})$ & 238.7 & 2.38 & 200.6 & 200 & 0.16 & 4000 \\
\hline (sc-g) & 19.53 & 1.02 & 38.3 & -- & -- & -- \\
\hline (sc-sn) & 19.53 & 1.02 & 38.3 & -- & -- & -- \\
\hline (sn-g) & 191.5 & 2.3 & 166.5 & -- & -- & -- \\
\hline Homogenized (H) & 127.8 & 2.03 & 125.9 & 256.8 & 0.202 & 2982 \\
\hline H-sc & 19.53 & 1.02 & 38.3 & -- & -- & -- \\
\hline H-sn & 127.8 & 2.03 & 125.9 & -- & -- & -- \\
\hline H-GB & 127.8 & 2.03 & 125.9 & -- & -- & -- \\
\hline
\end{tabular}




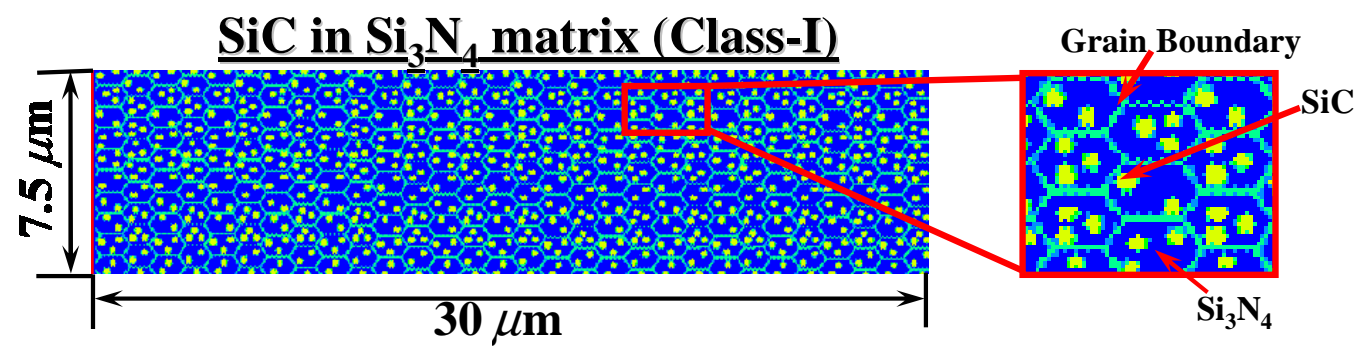

(a)

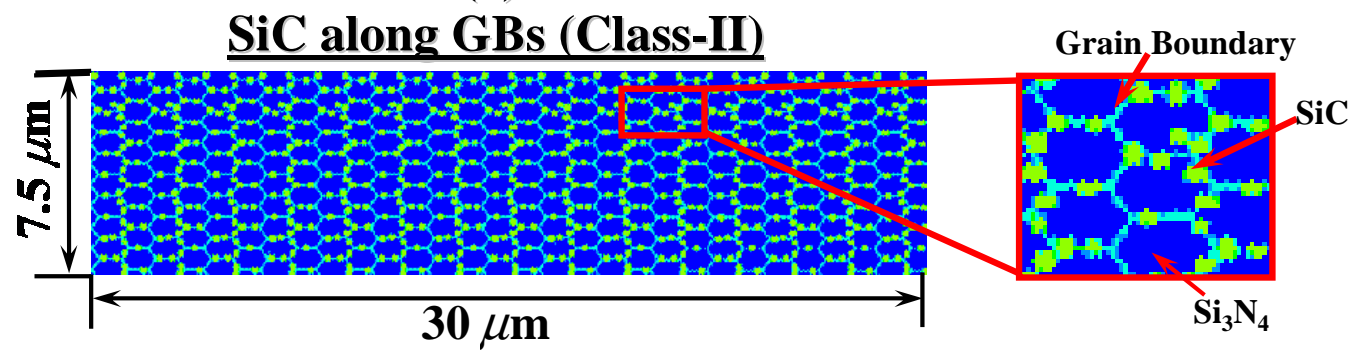

(b)

SiC along GBs and in $\mathrm{Si}_{3} \mathbf{N}_{4}$ matrix (Class-III) Grain,Boundary

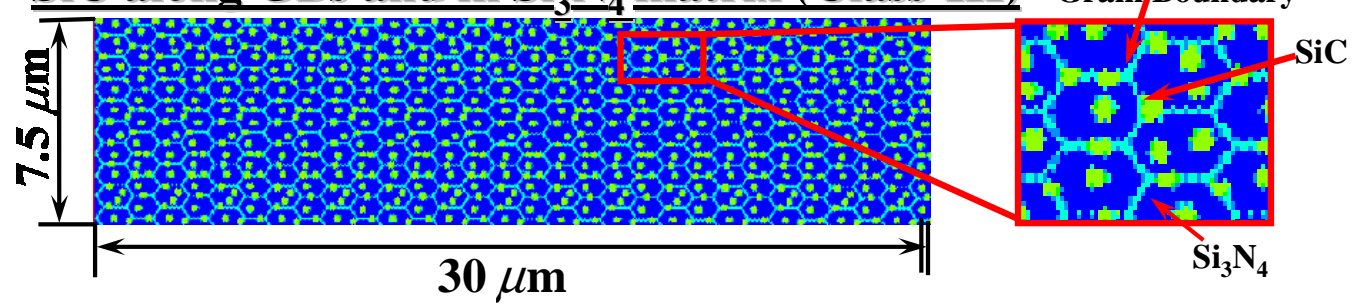

(c)

Figure 1 Microstructures analyzed with explicit resolution of GBs and second phase $\mathrm{SiC}$ particles. (a) Microstructure Class-I with $\mathrm{SiC}$ particles only in the $\mathrm{Si}_{3} \mathrm{~N}_{4}$ grain interiors, (b) Microstructure Class-II with SiC particles lying only along GBs, and (c) Microstructure ClassIII with $\mathrm{SiC}$ particles lying both along GBs and in $\mathrm{Si}_{3} \mathrm{~N}_{4}$ grain interiors (3 different variations of each microstructure shown in the figure are simulated in the reported analyses) 


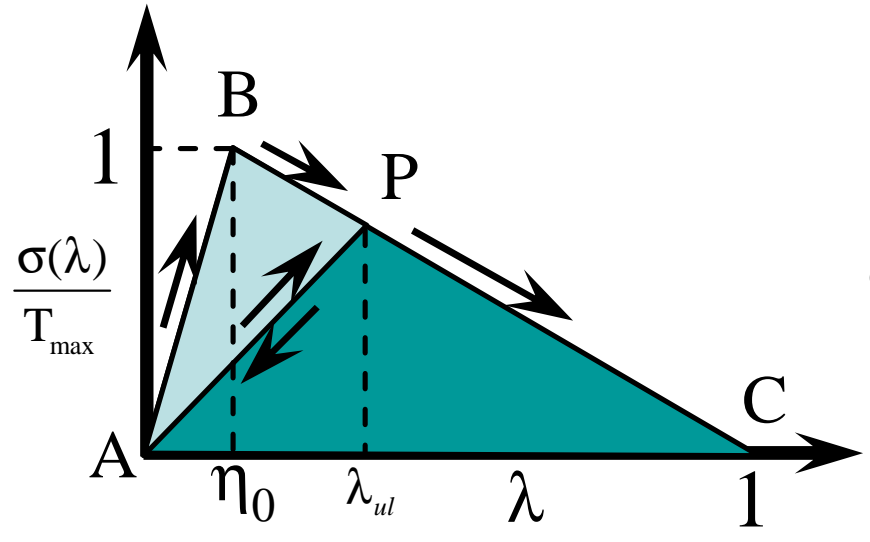

(a)

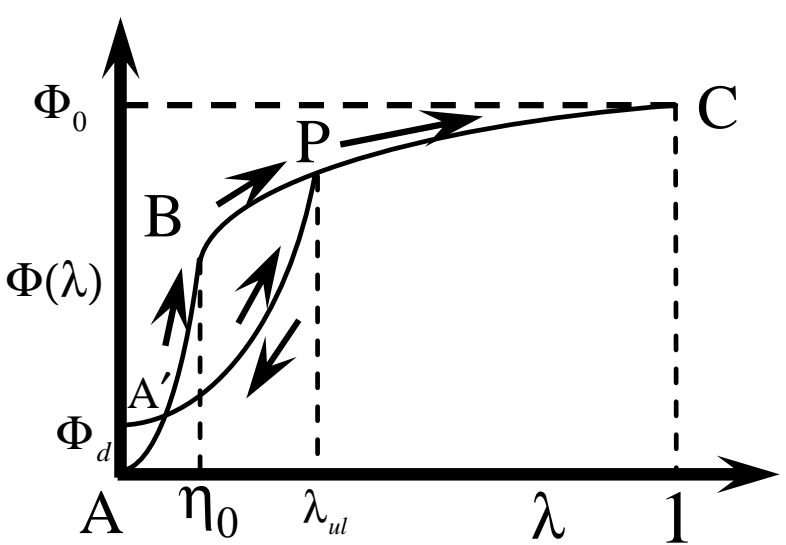

(b)

Figure 2 Irreversible bilinear cohesive law 


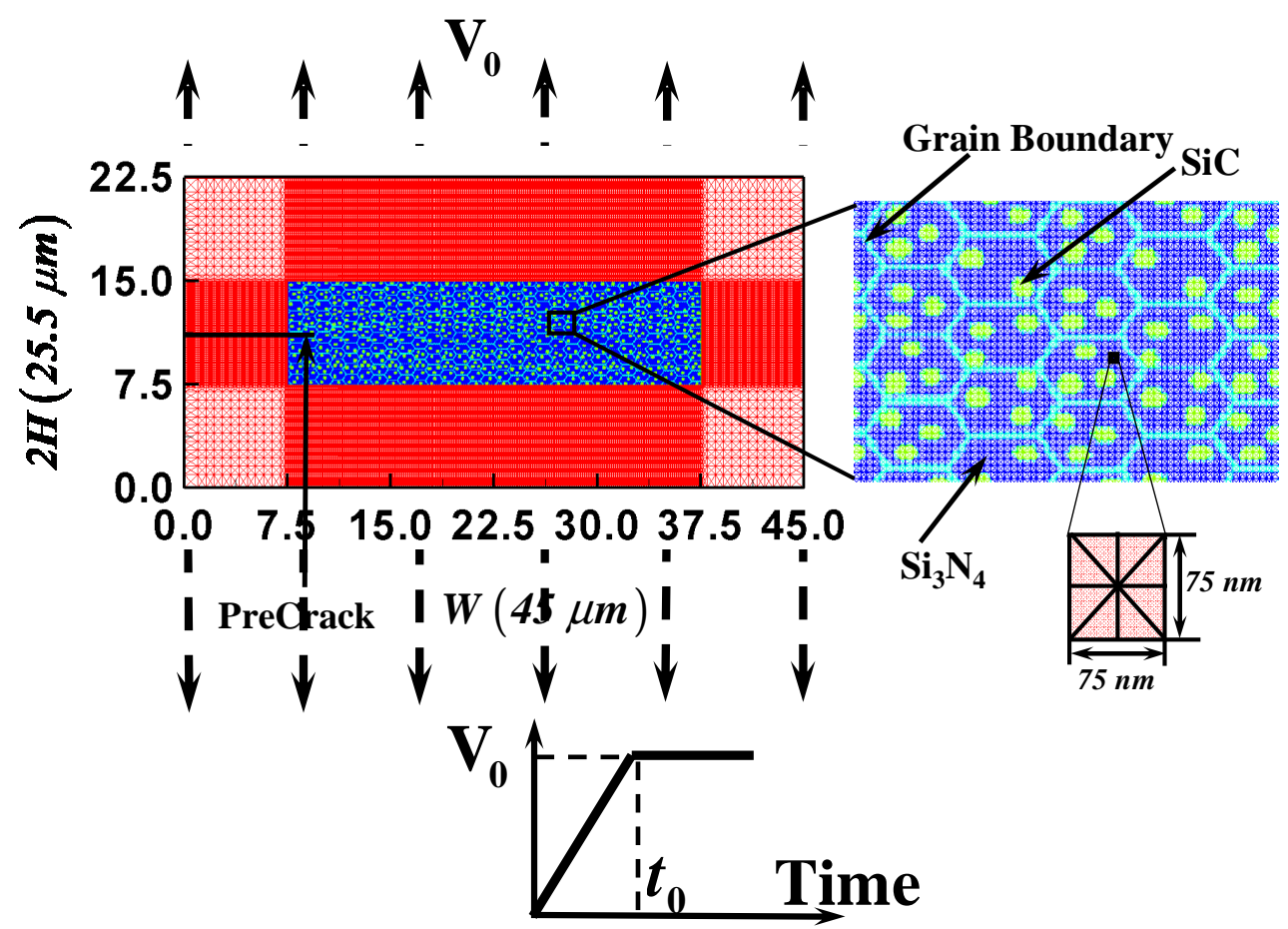

Figure 3 Dynamic fracture simulation setup with FEM discretization for performing the CFEM simulations 

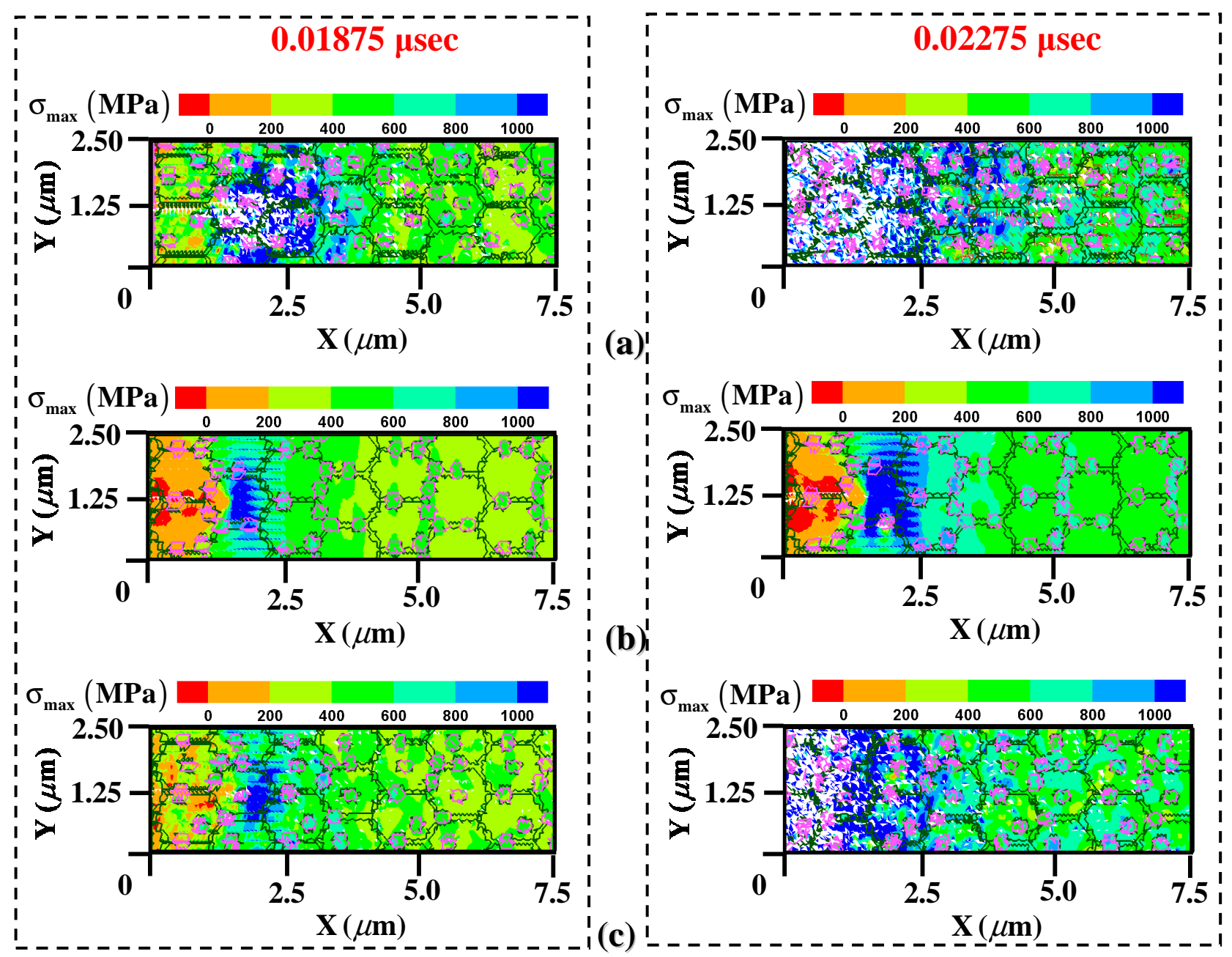

Figure 4 Stress distribution as a function of damage progression in (a) Class-I, (b) Class-II, and (c) Class-III microstructures at the loading rate of $2 \mathrm{~m} / \mathrm{sec}$ (plots show outline of the GB and second phase particles only in order to show the stress distribution. Corresponding broken particles have distorted "crumbled" appearance. The intact particles have near circular appearance. Corresponding fragmented GBs show up as zigzag lines in the diffused damage region. These lines are outlines of the fragmented GB elements.) 


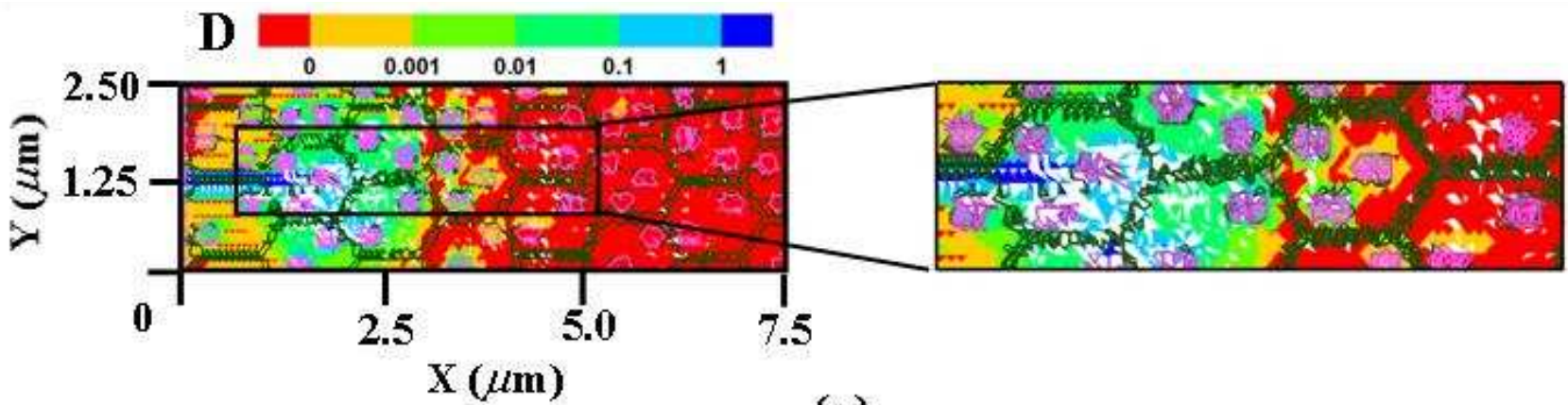

(a)

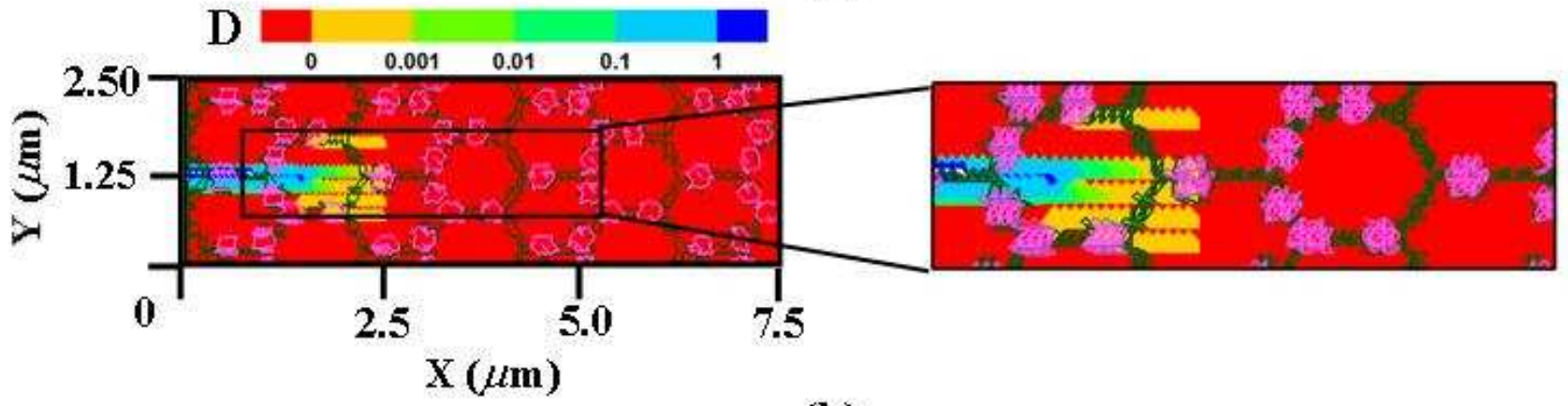

(b)

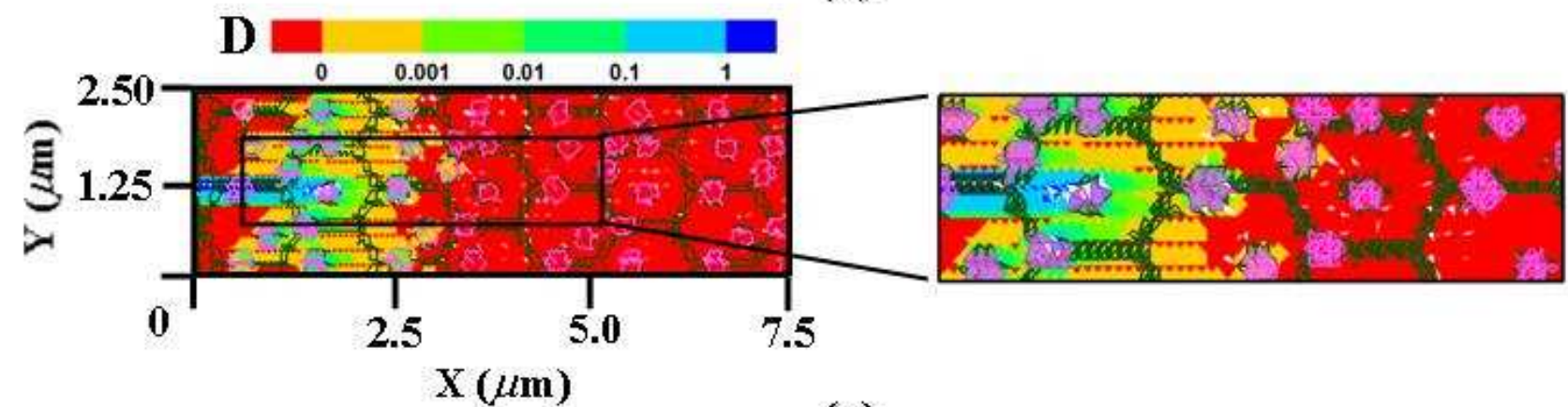

(c)

Figure 5 Damage distribution in (a) Class-I, (b) Class-II, and (c) Class-III microstructures at the loading rate of $2 \mathrm{~m} / \mathrm{sec}$ and time $t=0.01875 \mu \mathrm{sec}$. (closeup insets offer higher resolution view of the damage region. Broken particles have distorted "crumbled" appearance. The intact particles have near circular appearance. Corresponding fragmented GBs show up as zigzag lines in the diffused damage region. These lines are outlines of the fragmented GB elements.) 


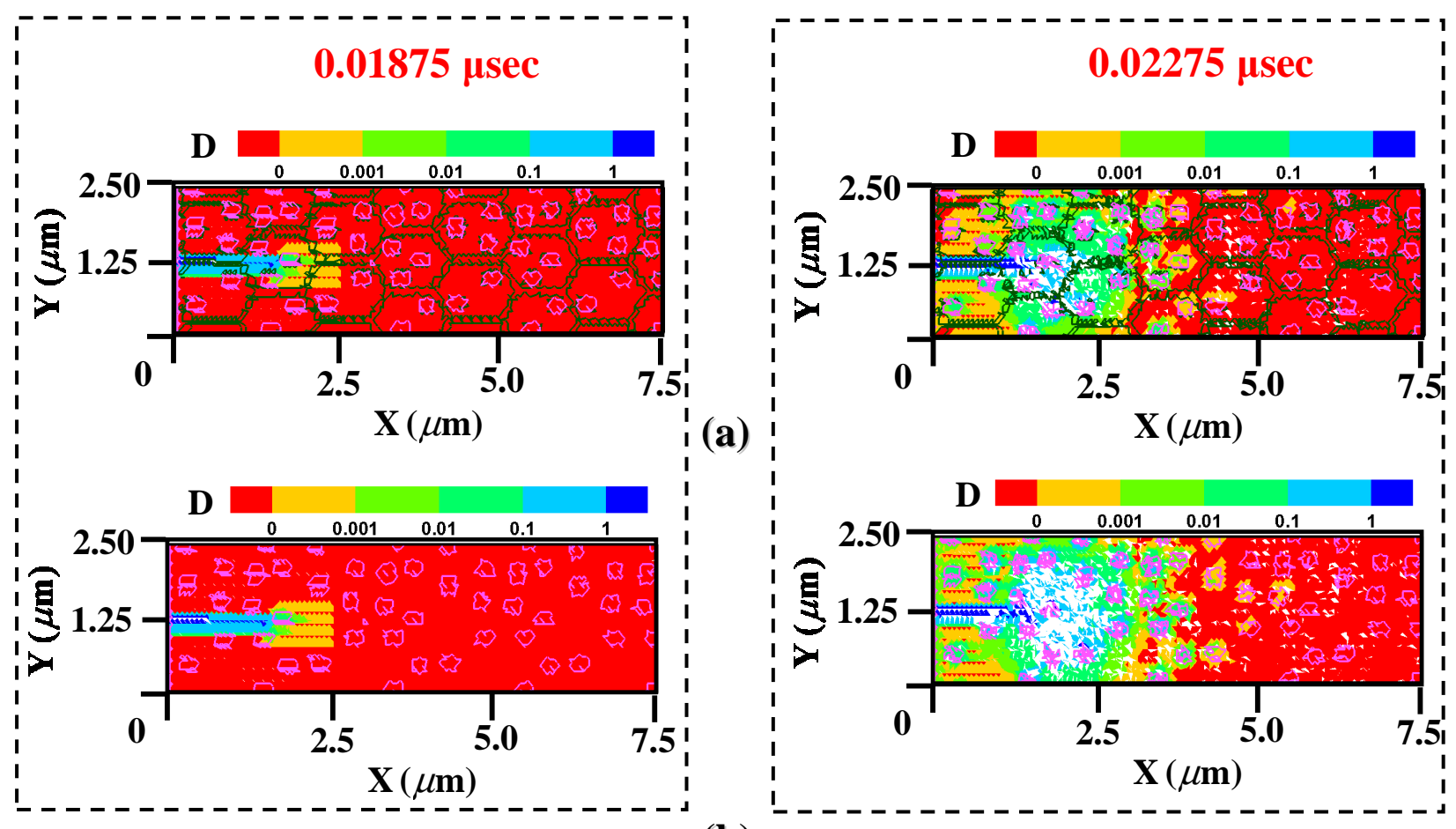

(b)

Figure 6 Progression of Damage distribution as a function of time in the case of (a) a Class-I microstructure and (b) in the case if GBs in class-I microstructure are removed at the loading rate of $2 \mathrm{~m} / \mathrm{sec}$. 


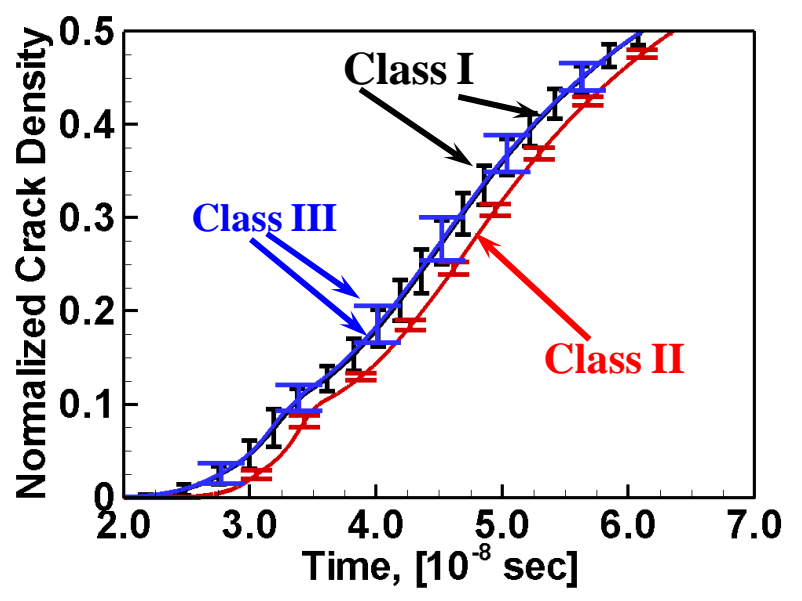

(a)

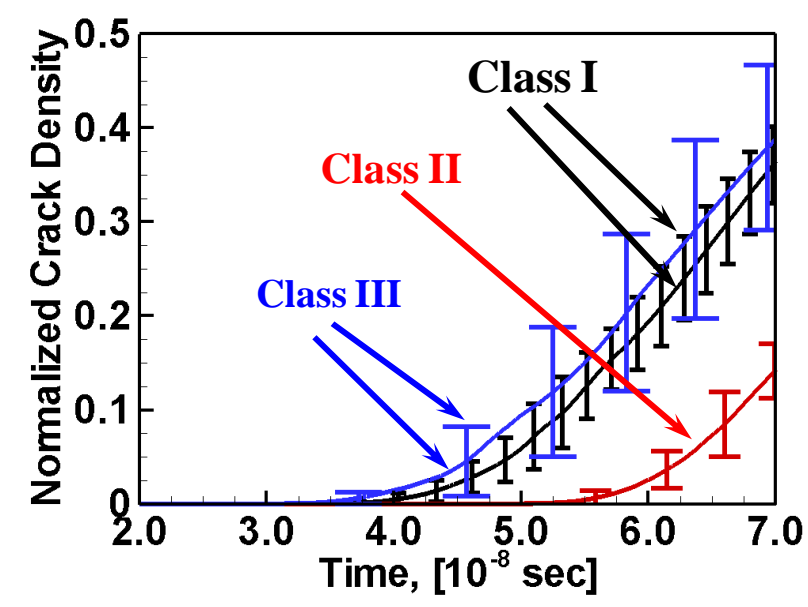

(b)

Figure 7 Mean and variations in normalized crack density values as a function of loading time values for microstructures at a loading rate of (a) $2 \mathrm{~m} / \mathrm{sec}$ and (b) $0.5 \mathrm{~m} / \mathrm{sec}$. 


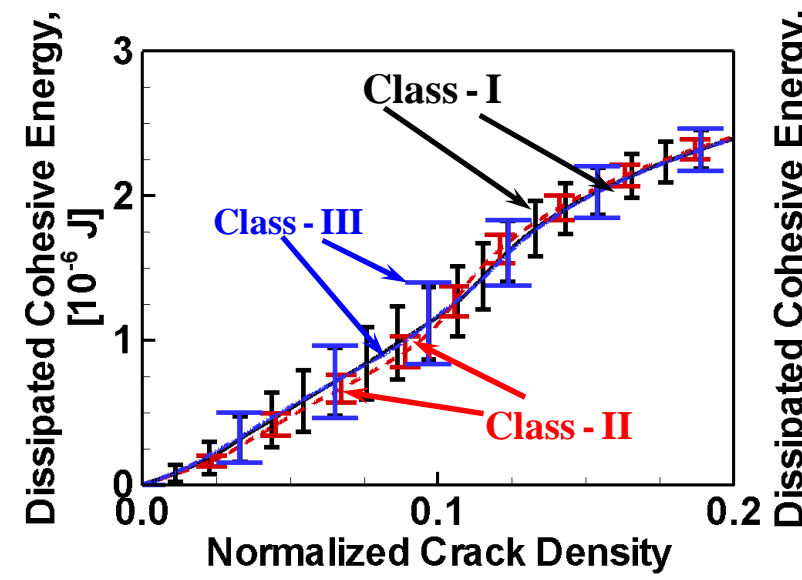

(a)

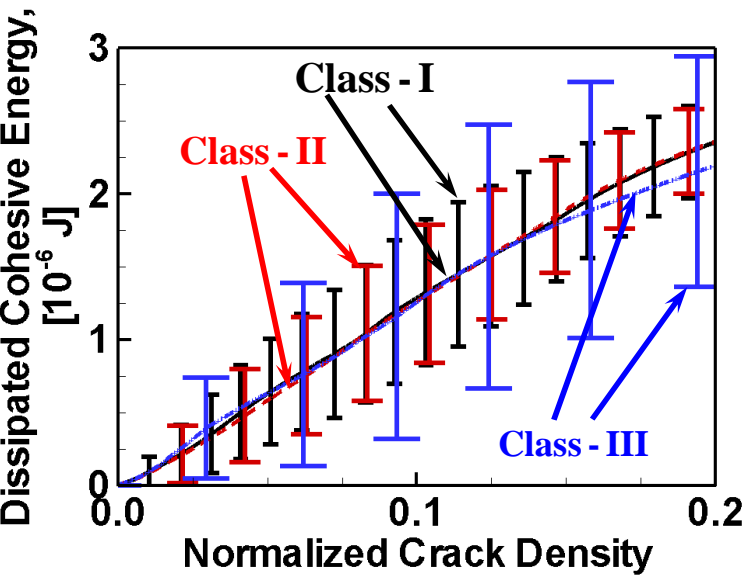

(b)

Figure 8 Mean and variations in dissipated cohesive energy values as a function of normalized crack density values for all microstructures at a loading rate of (a) $2 \mathrm{~m} / \mathrm{sec}$ and (b) $0.5 \mathrm{~m} / \mathrm{sec}$. 


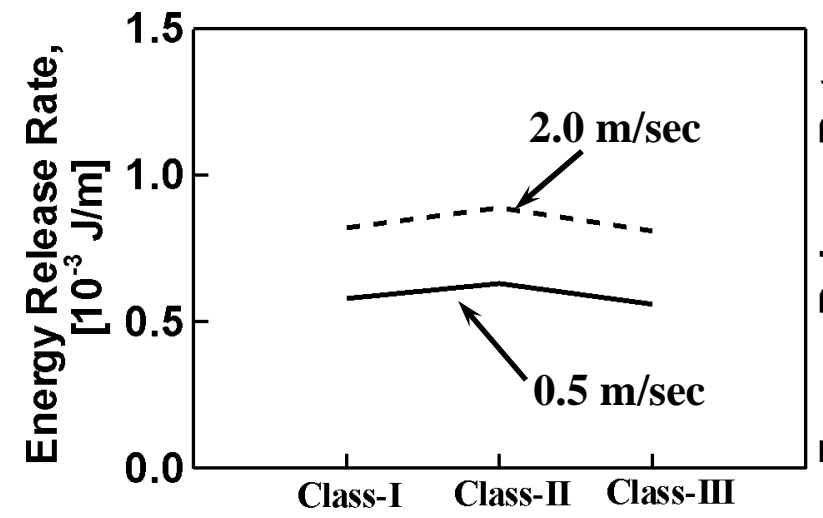

(a)

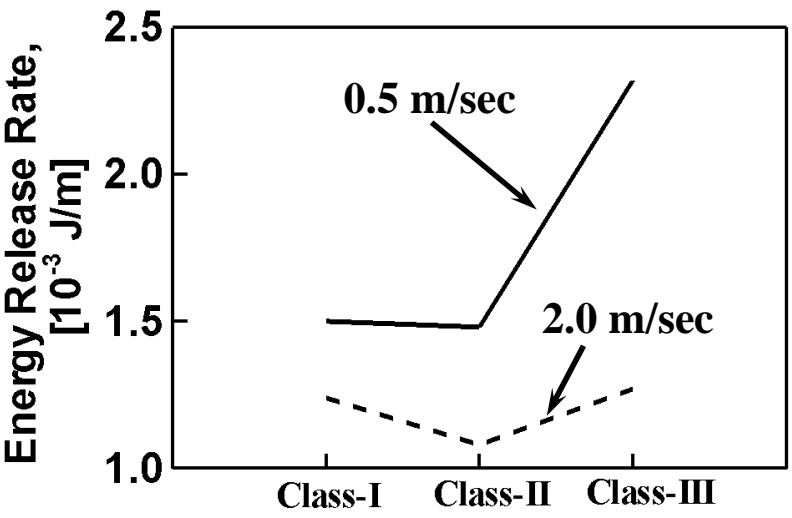

(b)

Figure 9 (a) Mean energy release rate values and (b) Maximum energy release rate values as a function of microstructure at the loading velocities of $2 \mathrm{~m} / \mathrm{sec}$ and $0.5 \mathrm{~m} / \mathrm{sec}$. 


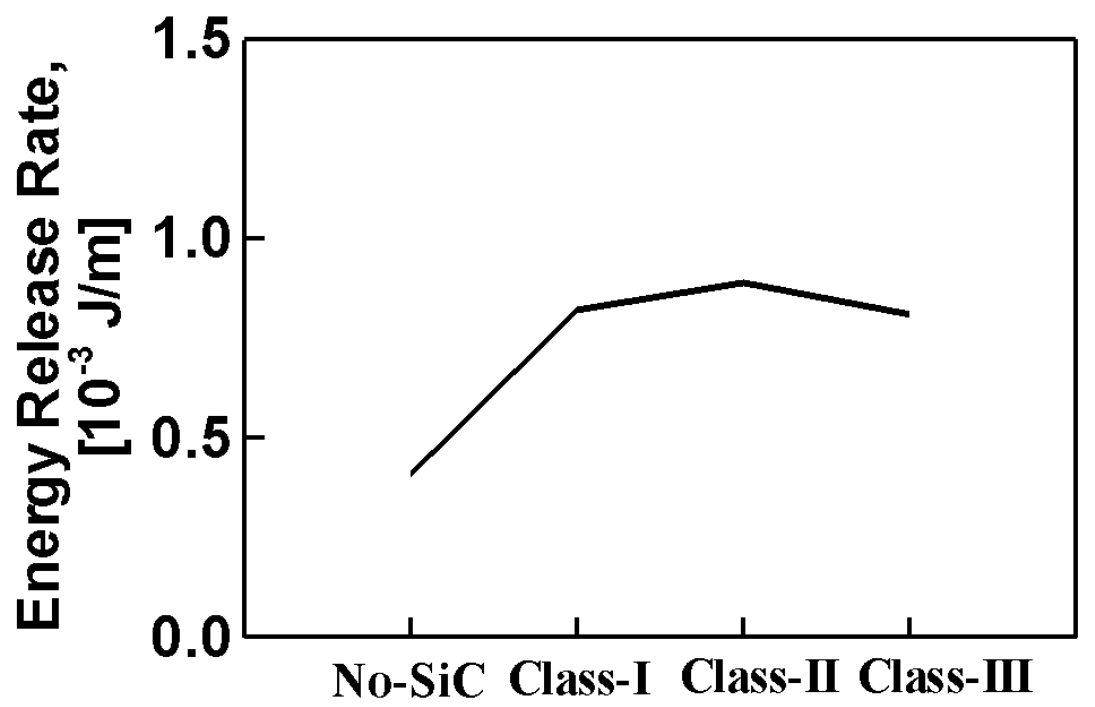

Figure 10 Mean energy release rate values as a function of microstructure at the loading velocity $2 \mathrm{~m} / \mathrm{sec}$ (No-SiC is a control microstructure with no $\mathrm{SiC}$ particles) 


\section{III-Molecular Modeling of Fracture in $\mathrm{SiC}_{-} \mathrm{Si}_{3} \mathrm{~N}_{4}$ Nanocomposites ${ }^{2}$}

Silicon Carbide ( $\mathrm{SiC})$-Silicon Nitride $\left(\mathrm{Si}_{3} \mathrm{~N}_{4}\right)$ nanocomposites with nano-sized $\mathrm{SiC}$ particles placed either in micro-sized $\mathrm{Si}_{3} \mathrm{~N}_{4}$ grains or along $\mathrm{Si}_{3} \mathrm{~N}_{4}$ grain boundaries (GBs) have been shown to be exceptionally strong at a range of temperatures, [1], [2], [3], [4], and [5]. However, a careful analysis of the effect of underlying morphology on the overall mechanical strength of this material system is lacking. A fundamental understanding of the effect of nano-sized SiC reinforcements on the mechanical behavior of these composites is required before further attempting to experimentally improve the properties by varied morphological alterations. Such studies exist for composites in which the reinforcement size is in the several-micron range, and in these studies, both the reinforcement size and volume fraction significantly affect the fracture toughness and mechanical strength, [42]. Recently, [43, 44] has analyzed the effect of morphological variations in second phase $\mathrm{SiC}$ particle placement and GB strength on the dynamic fracture strength of $\mathrm{SiC}-\mathrm{Si}_{3} \mathrm{~N}_{4}$ nanocomposites using continuum analyses based on a mesoscale cohesive finite element method (CFEM). It was found that the high strength and relatively small sized $\mathrm{SiC}$ particles act as stress concentration sites in $\mathrm{Si}_{3} \mathrm{~N}_{4}$ matrix leading to inter-granular $\mathrm{Si}_{3} \mathrm{~N}_{4}$ matrix cracking as a dominant failure mode. However, as a result of a significant number of nano-sized $\mathrm{SiC}$ particles being present in micro-sized $\mathrm{Si}_{3} \mathrm{~N}_{4}$ matrix, the $\mathrm{SiC}$

\footnotetext{
${ }^{2}$ A major part of this work has been published in International Journal with Citation "A. Tomar, V. and Gan, M., 2010, Temperature dependent nanomechanics of Si-C-N nanocomposites with an account of particle clustering and grain boundaries, in press in the Int. J. Hydrogen Energy, doi:10.1016/j.ijhydene.2010.03.070

B. Tomar, V., Gan, M., and Kim, H., 2010, Effect of temperature and morphology on mechanical strength of Si-C-O and Si-C-N nanocomposites, the Journal of European Ceramic Society, Volume 30, 2223-2237

C. Tomar, V., Samvedi, V., and Kim, H., 2010, Atomistic understanding of the particle clustering and particle size effect on the room temperature strength of SiC-Si3N4 nanocomposites to Appear in Int. J. Multiscale Comp. Engg. special issue on Advances In Computational Materials Science, volume 8 issue 4, 2010

D. Tomar, V., and Samvedi, V, 2009, Atomistic simulations based understanding of the mechanism behind the role of second phase $\mathrm{SiC}$ particles in fracture resistance of SiC-Si3N4 nanocomposites, Vol 7, issue 4, 277-294 pages, International Journal of Multiscale Computational Engineering"
} 
particles invariantly fall in wake regions of microcracks leading to significant mechanical strength. In this research, this mechanism is further examined using 3-D molecular dynamics (MD) simulations of $\mathrm{SiC}_{-} \mathrm{Si}_{3} \mathrm{~N}_{4}$ nanocomposite mechanical deformation. The focus is on analyzing the effect of particle clustering and particle size on the room temperature mechanical strength.

Classical MD replaces a comprehensive quantum mechanical treatment of interatomic forces with a phenomenological description in the form of an interatomic potential. MD has been used recently to achieve $\mathrm{nm} / \mathrm{cycle}$ fatigue crack extension rate similar to that observed in experiments, e.g. [45]. The MD simulation results of deformation twinning in 2-dimensional nanocrystalline Al with grain sizes from $30 \mathrm{~nm}$ to $90 \mathrm{~nm}$ by [46] have been found to be in close agreement with experimental observations reported by Liao and coworkers, [47], [48]. MD simulations have proven to provide good phenomenological trends on deformation mechanisms of nanocrystalline materials in agreement with experiments, e.g. [49] and [50]. Atomistic analyses of the nanocomposite mechanical strength as a function of phase morphology are relatively new and have focused on a very limited set of issues, e.g. [51-55]. Both $\mathrm{SiC}$ and $\mathrm{Si}_{3} \mathrm{~N}_{4}$ have been individually analyzed in the atomistic simulations for different mechanical strength related issues, e.g. $[56,57],[58,59]$. However, $\mathrm{SiC}_{-} \mathrm{Si}_{3} \mathrm{~N}_{4}$ nanocomposite morphologies are analyzed in this work for the first time. A range of $\mathrm{SiC}_{-} \mathrm{Si}_{3} \mathrm{~N}_{4}$ nanocomposite phase morphologies are generated with two different $\mathrm{SiC}$ particle sizes $(2 \mathrm{~nm}$ and $4 \mathrm{~nm}$ ) distributed in different manners in two different $\mathrm{Si}_{3} \mathrm{~N}_{4}$ phase matrices (one single crystalline and other bicrystalline).

\section{\$2. Formulation}

MD simulations are performed using a well established nanocomposite molecular dynamics simulations framework, [53, 60], under 3D periodic boundary conditions (PBCs). The focus is on 
understanding the deformation mechanisms and on delineating the factors affecting nanocomposite mechanical strength. In order to understand the effect of phase morphology on the nanocomposite mechanical strength, a range of microstructures are analyzed, e.g. Fig. 1. Because of the computational and space limitations only two different $\mathrm{SiC}$ particle sizes, $2 \mathrm{~nm}$ and $4 \mathrm{~nm}$, are considered. Computational limitations also resulted in the maximum $\mathrm{Si}_{3} \mathrm{~N}_{4}$ block size of $15 \times 15 \times 15 \mathrm{~nm}$ in all the microstructures. The imposition of PBCs results in the nanocomposites with regularly distributed pattern of second phase particles as illustrated in Fig. 1.

\section{$\S 2.1$ MD Framework}

The microstructures analyzed have 3-dimensional geometry. The microstructures are formed by placing $\mathrm{SiC}$ cylindrical particles in $\mathrm{Si}_{3} \mathrm{~N}_{4}$ blocks that have holes of sizes corresponding to the $\mathrm{SiC}$ particles. Setting up of the $\mathrm{SiC}-\mathrm{Si}_{3} \mathrm{~N}_{4}$ interface can critically affect the internal stresses in microstructures. In order to relieve any artificial internal stresses, first $\mathrm{Si}_{3} \mathrm{~N}_{4}$ blocks with the holes were prepared and equilibrated without the presence of the corresponding $\mathrm{SiC}$ cylindrical particles. $\mathrm{SiC}$ particles were separately equilibrated as clusters. After equilibration, the $\mathrm{Si}_{3} \mathrm{~N}_{4}$ block and the corresponding $\mathrm{SiC}$ particle were put together to form $\mathrm{SiC}$ $\mathrm{Si}_{3} \mathrm{~N}_{4}$ nanocomposites. Afterwards, the composite structures were again equilibrated. Such elaborate equilibration procedure was chosen so that the stress gradient at the $\mathrm{SiC}_{-} \mathrm{Si}_{3} \mathrm{~N}_{4}$ interface in the nanocomposites was at the minimum. We observed a large stress gradient at the $\mathrm{SiC}_{-} \mathrm{Si}_{3} \mathrm{~N}_{4}$ interface if the whole nanocomposite structure was equilibrated without separate equilibration of $\mathrm{SiC}$ inclusions and $\mathrm{Si}_{3} \mathrm{~N}_{4}$ matrix phase. A gap of $2 \AA$ was ensured between the $\mathrm{SiC}$ particles and the corresponding $\mathrm{Si}_{3} \mathrm{~N}_{4}$ block's hole in order to prevent additional buildup of the internal stresses. However, changing the gap did not influence the observed trends and results. MD 
equilibration period was determined based on convergence in energy values. Within the MD equilibration time period we did not any phase transformation due to particle-matrix interactions.

\section{\$2.2.1 Interatomic Potential for $\mathrm{SiC}-\mathrm{Si}_{3} \mathrm{~N}_{4}$ Material System}

Classical MD simulations of $\mathrm{Si}_{3} \mathrm{~N}_{4}+\mathrm{SiC}$ material systems require an interatomic potential to describe Si-Si, Si-N, Si-C, N-N, C-C, and N-C interactions. The potential should be fitted to the properties of $\mathrm{Si}_{3} \mathrm{~N}_{4}, \mathrm{SiC}$ and to an approximation to the interfacial transitions between these components. $\mathrm{Si}_{3} \mathrm{~N}_{4}$ family consists of two polymorphic members, $\alpha$ and $\beta$. The higher symmetry $\beta$ phase has a hexagonal lattice (space groups $C^{2}{ }_{6 h}, N 176$ ) with a primitive cell containing two $\mathrm{Si}_{3} \mathrm{~N}_{4}$ formula units $(\mathrm{a}=7.606 \AA, \mathrm{c}=2.909 \AA)$. The lower symmetry $\alpha$ phase is trigonal (space groups $\left.C_{3 v}^{4}, N 159\right)$ and has a primitive cell nearly twice as large (a=7.746 $\AA$, c=5.619 $⿱$ A) with twice as many atoms. The interatomic potentials for $\mathrm{Si}_{3} \mathrm{~N}_{4}$ material system have been developed by [61], [58], [62], [63], and [64]. Of all the above approaches, we choose the potential by [63] (based on Tersoff's potential) to model $\beta$ - $\mathrm{Si}_{3} \mathrm{~N}_{4}$ because of its simplicity and its ability to enable large scale MD simulations. $\beta$ - $\mathrm{Si}_{3} \mathrm{~N}_{4}$ is chosen because of its relative abundance in comparison to the $\alpha$ from. Different polytypes of $\mathrm{SiC}$ exist at ambient pressure, which are differentiated by the stacking sequence of the tetrahedrally bonded $\mathrm{Si}-\mathrm{C}$ bilayers, see [65]. Among these polytypes, $\beta$ $\mathrm{SiC}$ (cubic-SiC) is of much interest for its electronic properties. In particular, in recent years many theoretical and experimental studies have been carried out to investigate the different properties and possible applications of this material. Majority of interatomic potentials developed for $\mathrm{SiC}$ material system focus on describing the material properties of $\beta$-SiC, e.g. [66-68], [69], and [70]. We choose Tersoff's bond order potential to model interatomic interactions in SiC. At the interface of $\mathrm{SiC}$ and $\mathrm{Si}_{3} \mathrm{~N}_{4}$ we need to be able to describe $\mathrm{Si}-\mathrm{C}, \mathrm{Si}-\mathrm{Si}, \mathrm{C}-\mathrm{N}$, and $\mathrm{Si}-\mathrm{N}$ 
interactions. Tersoff's potential is useful only for describing the bulk Si-C, Si-Si, and Si-N interactions. We use potential developed for Carbonitrides by [71] to describe C-N interactions.

\section{\$2.2.2 High Performance Computing and Mechanical Deformation Algorithm}

\section{for MD Simulations}

MD simulations are performed using a modified version of a scalable parallel code, DL_POLY 2.14, [72] and [73]. The code has been modified and tested on a system of 1000000 atoms for a model ceramic matrix composite $\left(\mathrm{Al}+\mathrm{Fe}_{2} \mathrm{O}_{3}\right)$ material system and is benchmarked for scalable high performance classical MD simulations for large atomic ensembles with millions of atom. The simulations primarily focus on obtaining virial stress Vs. strain relations and visual atomistic deformation information in order to delineate the deformation mechanisms. In the uniaxial quasistatic deformation analyses of nanocrystalline $\mathrm{Cu}$ by [74], strain was calculated by recording the changes in positions of individual atoms. The average virial stress was calculated at every step in order to obtain the stress-strain relations. A modified version of this approach is used here. An alternative method to obtain uniaxial stress-strain curves is to record strain-time curves at several values of applied stress and then deduce the stress-strain relations, [75]. [76] have used both methods and found that the modified [77] method works better because it controls the applied strain and closely emulates controlled displacement experiments. The modifications to the method of [74] include the use of a combination of the algorithms for NPT and NVT ensembles. Alternating steps of stretching and equilibration at constant temperature are carried out to approximate uniaxial quasistatic deformation. Initially, the system is equilibrated at the specified temperature $(300 \mathrm{~K})$. During equilibration, NVT equations of motion are used to relax the pressure on structure in all three directions. During stretching, the MD computational cell is stretched in the loading direction using a modified version of the NPT equations of motion 
of [78]. NPT equations ensure that the structure has lateral pressure relaxed to atmospheric values during deformation. In this algorithm, the rate of change of a simulation cell volume, $V(t)$, is specified using a barostat friction coefficient parameter $\eta$ such that

$$
\frac{d \eta(t)}{d t}=\frac{1}{N k_{B} T_{\text {ext }} \tau_{P}^{2}} V(t)\left(P-P_{e x t}\right)
$$

and,

$$
\frac{d V(t)}{d t}=3 \eta(t) V(t)
$$

Here, $P$ is the instantaneous pressure, $P_{\text {ext }}$ is the externally applied pressure, $N$ is total number of atoms in the system, $k_{B}$ is the Boltzmann constant, $T_{e x t}$ is the external temperature, and $\tau_{P}$ is a specified time constant for pressure fluctuations. For a given cross-sectional area, the specification of $\eta$ in Eq. (12) is equivalent to specifying strain rate for the change in simulation cell length. Further, for a given $\eta$, Eq. (11) can be modified as,

$$
\frac{d \eta(t)}{d t}=\frac{1}{N k_{B} T_{e x t} \tau_{P}^{2}} V(t)\left(P-P_{e x t}\right)-\gamma \eta
$$

In the above equation, the term $\eta \gamma$ acts as a damping coefficient for reducing fluctuations in pressure during the stretching of the simulation cell. During the simulations, the system is initially equilibrated at $T_{\text {ext }}=300 \mathrm{~K}$. After equilibration, the computational cell is stretched in the loading direction using $\eta=0.1 \mathrm{ps}^{-1}$. This results in a tensile uniaxial stress loading in the $\mathrm{x}$ direction, while the y- and z-directions have atmospheric stresses imposed upon the system. In general, MD's time step limitations make it unsuitable to be used as a predictive technique for performing small strain rate mechanical deformation simulations. $\eta=0.1 \mathrm{ps}^{-1}$ corresponds to a very high strain rate. This strain rate is a limitation of MD as a method and results of the MD 
simulations need to be interpreted while keeping this in mind. The values of $\gamma=0.5$ and $P_{\text {ext }}=1$ atmospheric pressure are used. The values for $\eta$ and $\gamma$ are calculated in trial calculations that focused on achieving the best balance between simulation time (low $\eta$ results in long simulation times and vice-versa) and pressure fluctuations (high $\gamma$ results in excessive pressure damping with increase in residual stresses along periodic boundaries). In the analyses reported in the manuscript, the MD equilibration time in between the periods of stretching is chosen as $2.0 \mathrm{ps}$. The equilibration time for $2 \mathrm{ps}$ is chosen such that the total potential energy fluctuation of the system with respect to time is minimized. The change in time from $2 \mathrm{ps}$ to longer did not change the simulation results. Keeping in mind the limitations of MD simulations in terms of timesteps we did not see any benefit in using the increased equilibration period. The simulation timestep is 1 fs. The total time for each stretching step was approximately 3 ps.

\section{$\$ 3$ Mechanical Deformation Analyses}

MD results are analyzed using visuals observations and analyzing virial stress-true strain relations. Virial stress represents internal resistance offered by a material to an externally imposed load, [79]. Since virial stress is an internal quantity, it is also an indicator of the increase in the stress concentration caused by the presence of an inhomogenity in an otherwise homogenous body. This characteristic is used to analyze the effect of the second phase particles in the presented analyses. The focus of the analyses is on developing an understanding of the correlations between the particle size, the particle placement with respect to a bicrystalline interface, and the particle clustering. For this purpose viewgraphs showing deformation mechanisms are also plotted as a function of tensile displacements.

Figure 1 shows the first set of microstructures analyzed. $\mathrm{S} 1$ is a block of $\mathrm{Si}_{3} \mathrm{~N}_{4}$ with $\mathrm{S1}_{2}$ having $2 \mathrm{~nm} \mathrm{SiC}$ particles and $\mathrm{S}_{4}$ having $4 \mathrm{~nm} \mathrm{SiC}$ particles uniformly dispersed in the middle. 
$\mathrm{S} 2$ is a bi-crystalline $\mathrm{Si}_{3} \mathrm{~N}_{4}$ block with $\mathrm{S} 2_{2}$ having $2 \mathrm{~nm} \mathrm{SiC}$ particles and $\mathrm{S} 2_{4}$ having $4 \mathrm{~nm} \mathrm{SiC}$ particles uniformly dispersed in the middle. Crystalline orientations in S2 are shown in Fig. 1. $\mathrm{SiC}$ particles in all atomistic morphologies have their (001) surface perpendicular to the plane of the figures. Figure 2 shows virial stress-strain plots for the microstructures shown in Fig. 1. Microstructure S1 is found to be the strongest of all the microstructures. Presence of second phase particles reduces strength of S1 with larger particle size resulting in higher reduction. Microstructure S2 has considerably less strength than S1 with the difference being the presence of bicrystalline boundary in S2. Presence of second phase particles does not have an appreciable effect on the strength of microstructure S2. It is normally accepted that particle placement along interface has strong effect on the composite strength. However, the result for microstructure S2 is counterintuitive since the presence of particles does not influence the nanocomposite strength. It is important to correlate these findings with the visuals of mechanical deformation in order to understand the underlying mechanism behind these findings.

Figure 3 shows the viewgraphs of mechanical deformation of the microstructures $\mathrm{S} 1, \mathrm{S1}_{2}$, and $\mathrm{S} 1_{4}$. A comparison of the viewgraphs reveals that the primary mechanism responsible for lowering of the strength during nanocomposite deformation is ductile shearing at the $\mathrm{SiC}$ and $\mathrm{Si}_{3} \mathrm{~N}_{4}$ interfaces. The most likely cause of the shearing can be the mismatch between the elastic moduli of $\mathrm{SiC}$ and $\mathrm{Si}_{3} \mathrm{~N}_{4}$. This results in $\mathrm{SiC}$ particle deforming at a different rate than $\mathrm{Si}_{3} \mathrm{~N}_{4}$ matrix leading to the deformation discontinuity at the $\mathrm{SiC}_{-} \mathrm{Si}_{3} \mathrm{~N}_{4}$ interface. Ductile shearing is higher for microstructure with higher particle size leading to higher reduction in mechanical strength. In order to understand the effect of interface on mechanical strength, viewgraphs of mechanical deformation of the microstructures $\mathrm{S} 2, \mathrm{~S} 2_{2}$, and $\mathrm{S} 2_{4}$ are shown and analyzed in Fig. 4. As shown, ductile shearing at the $\mathrm{SiC}$ and $\mathrm{Si}_{3} \mathrm{~N}_{4}$ interfaces is still present. However, an additional 
deformation mechanism is ductile shearing of the upper crystalline block in all the S2 microstructures. A closer examination revealed that the additional mechanism has stronger effect on the mechanical strength leading to the particle placement having no effect on the mechanical strength of the microstructures $\mathrm{S} 2, \mathrm{~S} 2{ }_{2}$, and $\mathrm{S} 24$.

Earlier, crack propagation in the $\mathrm{SiC}-\mathrm{Si}_{3} \mathrm{~N}_{4}$ nanocomposites is found to be strongly affected by the presence of second phase particles and the interfaces, $[43,44]$. In order to understand the interaction of crack with second phase $\mathrm{SiC}$ particles and interface, crack propagation is microstructures $\mathrm{S} 2_{2}$ and $\mathrm{S} 2_{4}$ is modeled using the methodology of [49]. A crack of initial length $1.5 \mathrm{~nm}$ is introduced in both microstructures by switching off interatomic interactions in the crack plane. Figure 5 compares the stress-strain plot for microstructures $\mathrm{S} 2_{2}$ and $\mathrm{S} 2{ }_{4}$ with and without the presence of initial crack. The plots don't show any appreciable difference indicating that the presence of initial crack at the length scale of study does not appreciably affect the mechanical strength. This directly indicates the presence of flaw tolerance at the length scale of study in the nanocomposites analyzed. In order to verify this, viewgraphs of different mechanical deformation stages for the two structures are shown in Fig. 6. As shown, the crack propagates in a brittle manner in the structure. However, ductile shearing at the $\mathrm{SiC}_{-} \mathrm{Si}_{3} \mathrm{~N}_{4}$ interfaces and in upper crystalline block in the two structures is quite similar to that shown in Fig. 4. The presence of crack does not alter the deformation mechanism verifying the existence of flaw tolerance at the length scale of study.

From the above analyses and findings, it is clear that the presence of crack does not affect the mechanical strength at the length scale of study. Accordingly, crack propagation is not analyzed further in any structure. From the above findings, it is clear that for the bicrystalline structure S2 particle placement at the interface does not affect its mechanical strength. One significant 
question that arises is whether the difference could be brought about by changing the relative position of particles with respect to each other at the interface? In order to answer such question, a second set of microstructures as shown in Fig. 7 were generated with 4 different variations in the particle placements. The difference between $S 4_{2}$ and $S 5_{2}$ as well as that between $S 6_{2}$ and $S 7_{2}$ in Fig. 7 (a) is that in $\mathrm{S}_{2}$ and $\mathrm{S}_{2}$ particles do not touch the interface. While in $\mathrm{S}_{2}$ and $\mathrm{S} 7_{2}$ the particles touch the interface. Similar sets of arrangements are shown as $\mathrm{S} 4_{4}, \mathrm{~S}_{4}, \mathrm{~S}_{4}$, and $\mathrm{S}_{2}$ in Fig. 7 (b). Fig. 8 shows the stress-strain plots for the microstructures in Fig. 7 and compares the results with those for $\mathrm{S} 2_{2}$ and $\mathrm{S} 24$. As shown, the changes in the particle placement pattern do not alter the mechanical strength. This finding also reinforces earlier view that the sharing of upper crystalline block is the most dominant mechanism in bi-crystalline microstructures analyzed in the present investigation.

Another important aspect of the particle placement effect on mechanical strength is the effect of clustering of the particles on the mechanical strength. In order to understand this aspect, a third set of microstructures is generated as shown in Fig. 9. As shown, $\mathrm{S}_{2}$ and $\mathrm{S}_{4}$ have $2 \mathrm{~nm}$ and $4 \mathrm{~nm}$ sized $\mathrm{SiC}$ particles distributed around the bi-crystalline interface, respectively. Similarly, $\mathrm{S} 9_{2}$ and $\mathrm{S} 9_{4}$ have $2 \mathrm{~nm}$ and $4 \mathrm{~nm}$ sized $\mathrm{SiC}$ particles distributed in the middle, respectively. As shown in Fig. 10, the particle clustering reverses the earlier findings shown in Fig. 2. As shown in Fig. 10 (a) placing particles in clusters around bi-crystalline interface results in significant rise in mechanical strength with higher strength being attributed to smaller particle size. Lowering of the strength for higher particle size can be attributed to higher extent of the corresponding $\mathrm{SiC}_{-} \mathrm{Si}_{3} \mathrm{~N}_{4}$ ductile shearing. As shown in Fig. 10 (b), the clustering significantly reduces the mechanical strength of a $\mathrm{Si}_{3} \mathrm{~N}_{4}$ block. However, now the particle size has insignificant effect on the mechanical strength. 
Overall, combination of observations and findings indicate that bi-crystalline orientation, particle size, particle placement, and particle clustering are important factors that need to be taken into account while accounting for nanocomposite mechanical strength. The toughening of the nanocomposite in the presence of a combination of interfaces and second phase particles is strongly dependent upon relative second phase particle arrangement. The nanocomposite strengthening due to the second phase is strongly dependent upon the size and relative spacing between particles irrespective of whether the particles are in grain interior or at the interfaces. Continuum averaging relations as well as continuum numerical methods such as micromechanical finite element methods that neglect finer length scale phase morphology need to be refined to account for such important factors.

\section{§4 Conclusions}

In the presented work MD based analyses to understand the effect of second phase $\mathrm{SiC}$ particle arrangement on the strength of $\mathrm{SiC}_{-} \mathrm{Si}_{3} \mathrm{~N}_{4}$ nanocomposites are presented. Earlier continuum analyses using micromechanical finite element models have shown that second phase particles can be effective in increasing the nanocomposite strength. However, such analyses suffer from fundamental size effect limitations and cannot analyze particle size effect at finer length scales on the nanocomposite mechanical strength. In this scenario, the analyses presented in the current work offer an attractive avenue. Focus of the presented work is on understanding how the particle placement and clustering can be performed to make a strong morphology. The crack path resistance could not be analyzed because of the existence of fault tolerance at the length scale of study. However, simple mechanical strength analyses offered good insights into the phase morphology effect on microstructural strength. Analyses indicated that the second phase particles act as significant stress raisers in the case of single crystalline $\mathrm{Si}_{3} \mathrm{~N}_{4}$ phase matrix 
reducing the mechanical strength by a factor of 1.8 with higher particle size causing larger reduction in peak strength. However, the particle's presence does not have any effect on the mechanical strength of bicrystalline $\mathrm{Si}_{3} \mathrm{~N}_{4}$ phase matrix. Deformation mechanism consists of considerable ductile shearing at the $\mathrm{SiC}_{-} \mathrm{Si}_{3} \mathrm{~N}_{4}$ interfaces in all structures. Presence of initial crack has very little effect on the overall mechanical strength, indicating the presence of flaw tolerance at the length scale of analyses. An examination of the effect of particle clustering together in $\mathrm{Si}_{3} \mathrm{~N}_{4}$ phase matrix showed that particle clusters with larger particle size result in higher reduction in mechanical strength. Using smaller particles led to greater strength enhancement. One of the causes of such inclusion size effect on nanocomposite strength should be change in the elastic stiffness of inclusion with smaller being stiffer. However, we would also like to point out that $\mathrm{SiC}$ inclusions taken alone and taken in a matrix are two different things. This is because in the matrix we would also have interfacial interactions at the $\mathrm{SiC}_{-} \mathrm{Si}_{3} \mathrm{~N}_{4}$ interfaces. This implies that the effect of inclusion size on the nanocomposite strength may also have been caused by reduced stress concentration with smaller particles.

Overall, the result pointed out that the placement of $\mathrm{SiC}$ particles along the interfacial boundaries will not always lead to strengthening of a $\mathrm{SiC}_{-} \mathrm{Si}_{3} \mathrm{~N}_{4}$ nanocomposite. MD analyses confirm the earlier CFEM findings of $[43,44]$ concerning the effect of second phase SiC particles on the $\mathrm{SiC}-\mathrm{Si}_{3} \mathrm{~N}_{4}$ nanocomposite strength. In addition, analyses also point out that the strengthening of the nanocomposite by placing second phase particles along grain boundaries is only possible for a selective few second phase particle sizes with inter-particle spacing and particle clustering being very important factors. At the simulation length scale we observed flaw-tolerance. Therefore, we cannot model the crack path resistance. However, by increasing the length scale of the simulations crack path resistance analyses are possible. One way to use 
the presented simulations in modeling higher length scale crack path resistance behavior is to use the results for predicting the maximum interfacial strength and its variation as a function of nanoscale morphology effects. The trends and maximum strength values could be supplied in higher length scale models such as the cohesive finite element method (e.g. [43, 44]) that could predict the crack path resistance while considering the nanoscale morphology effects. 

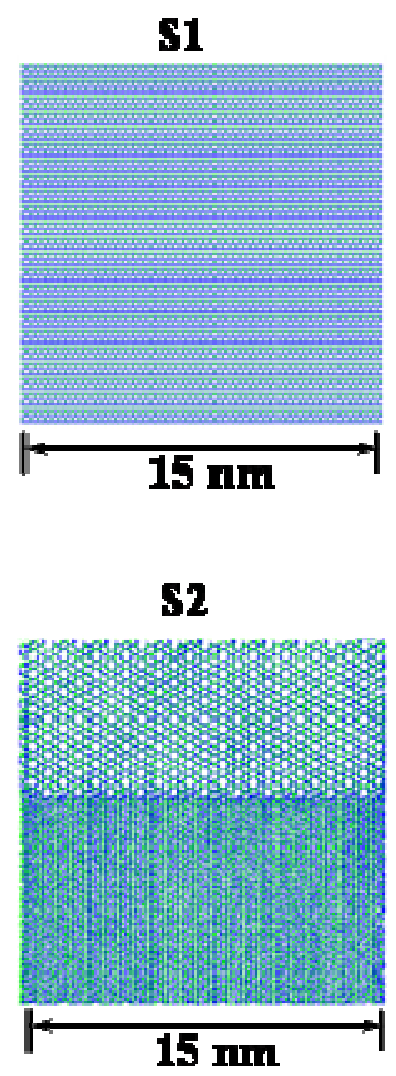

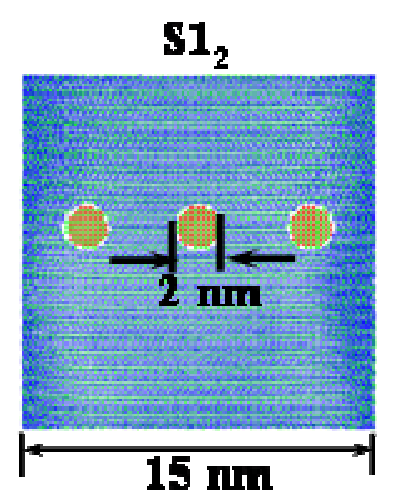

(a)

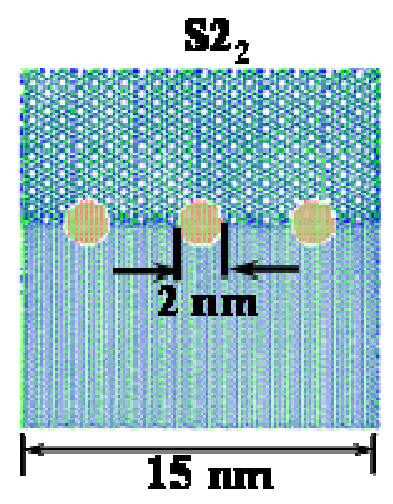

(b)
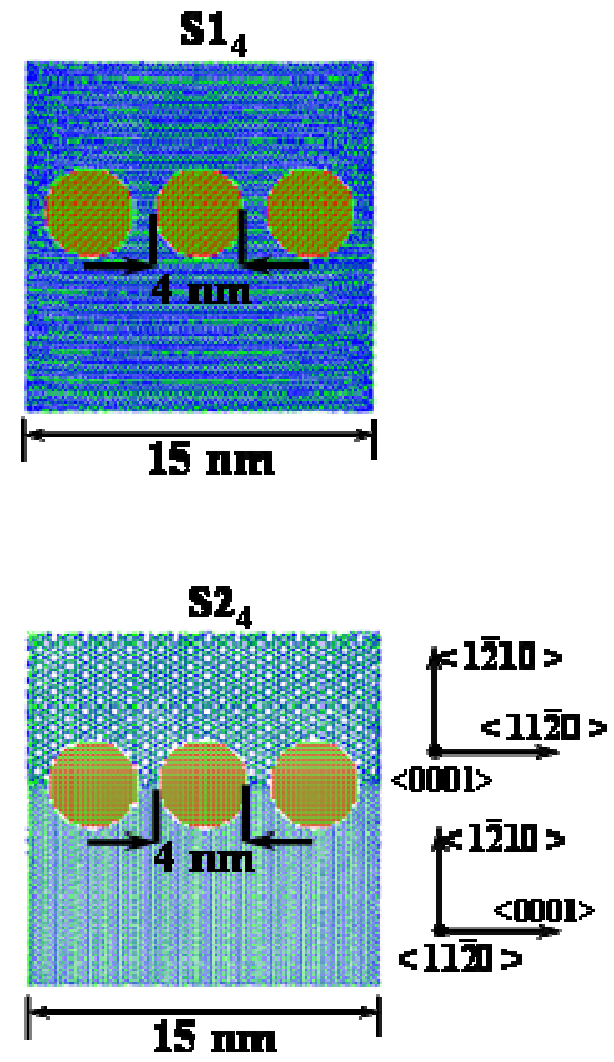

Figure 1 First set of atomistic microstructures analyzed 


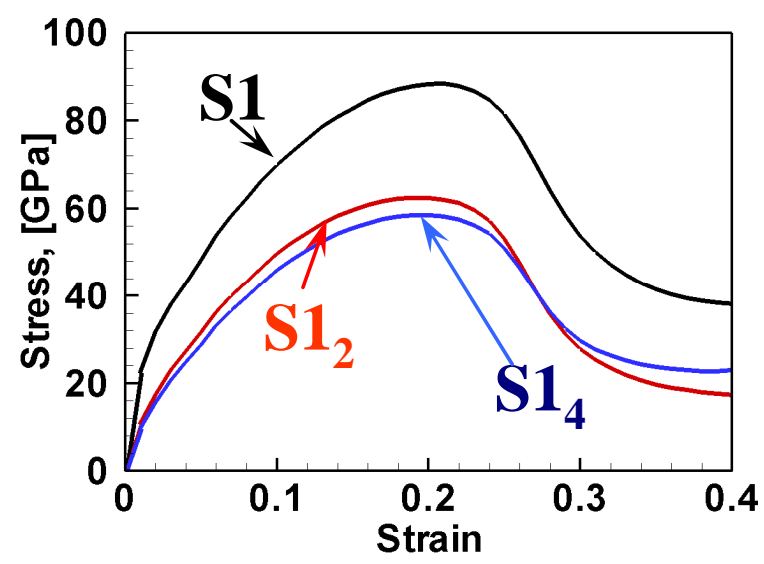

(a)

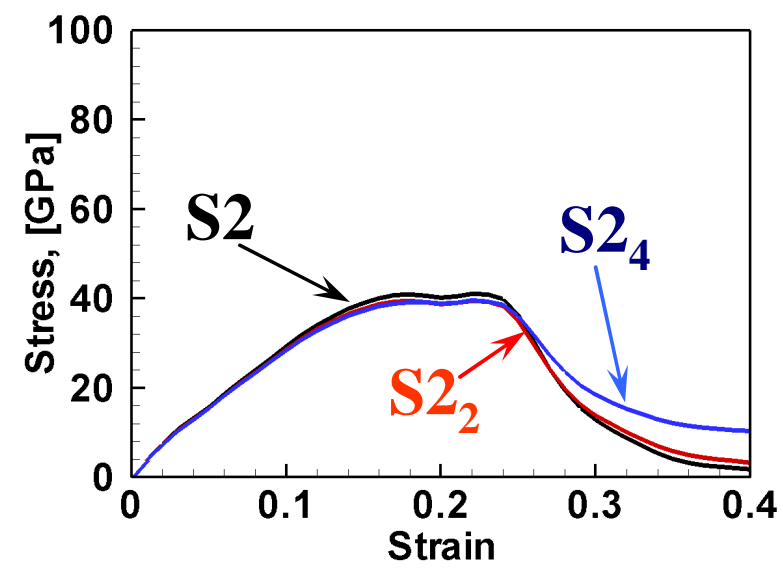

(b)

Figure 2 Virial stress in the loading direction Vs. uniaxial true strain plots for (a) microstructures $\mathrm{S} 1, \mathrm{~S} 1_{2}$, and $\mathrm{S} 1_{4}$ and (b) for microstructures $\mathrm{S} 2, \mathrm{~S} 2_{2}$, and $\mathrm{S} 2_{4}$ 

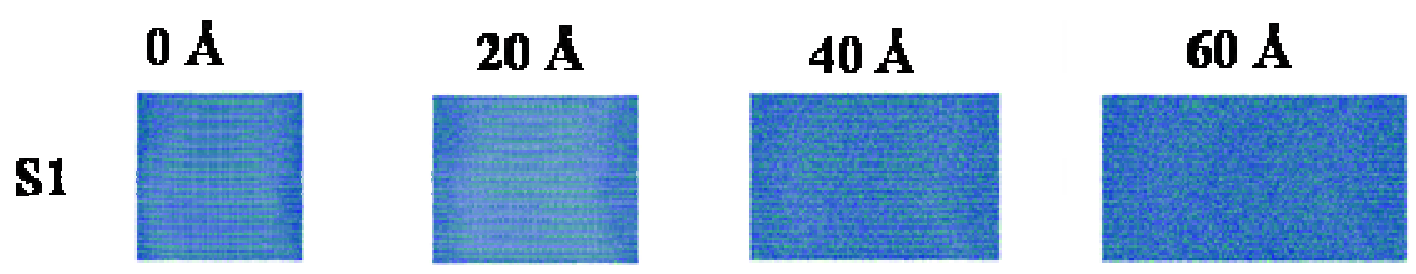

80 A
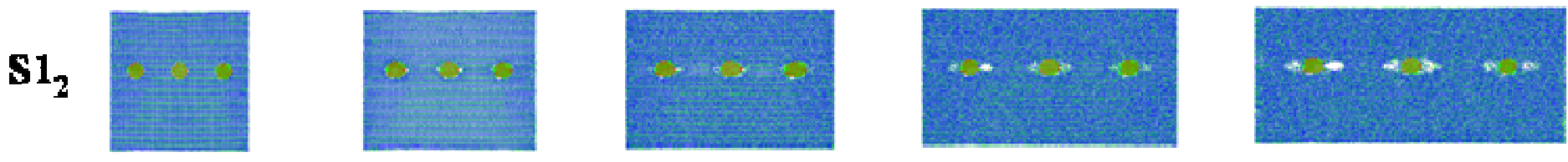

$\mathbf{S} 1_{4}$
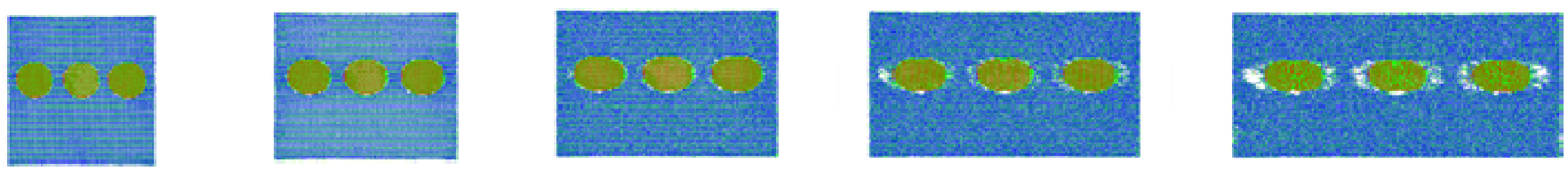

Figure 3 Viewgraphs showing various stages of deformation in $\mathrm{S} 1, \mathrm{~S}_{2}$, and $\mathrm{S} 1_{4}$. The displacement shown is the tensile deformation as a result of applied uniaxial strain rate on the nanocomposites 


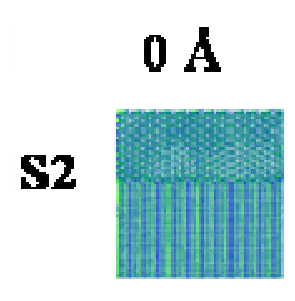

20 A
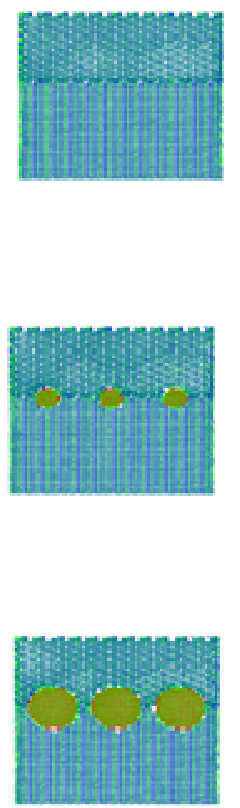

$40 A$
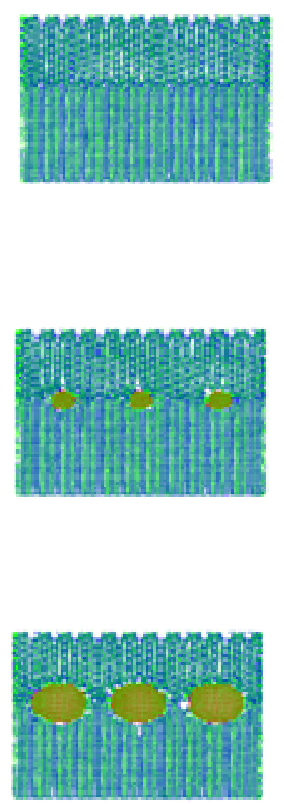

$60 A$
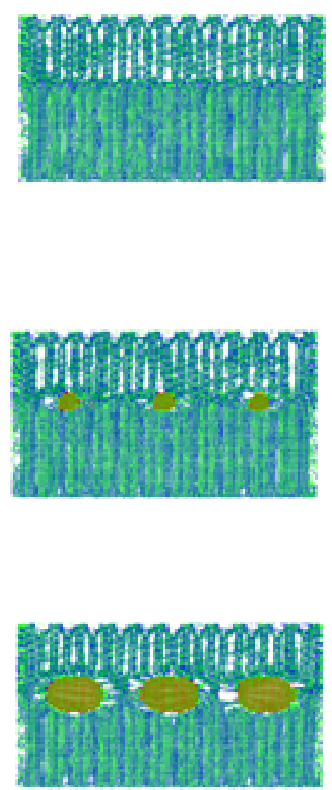

80 A
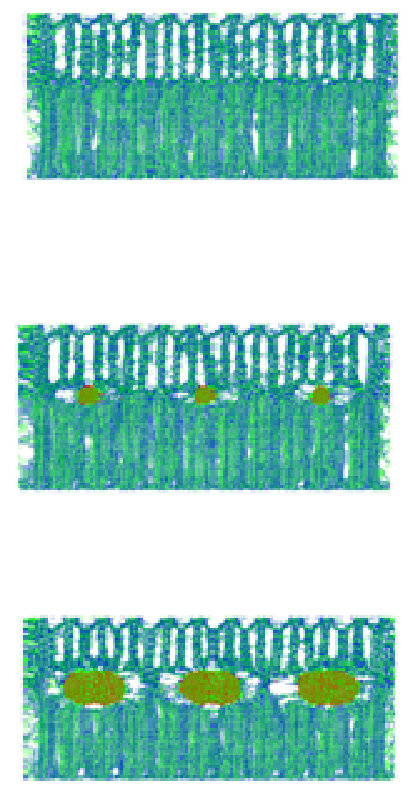

Figure 4 Viewgraphs showing various stages of deformation in $\mathrm{S} 2, \mathrm{~S} 22_{2}$, and $\mathrm{S} 24$. The displacement shown is the tensile deformation as a result of applied uniaxial strain rate on the nanocomposites 


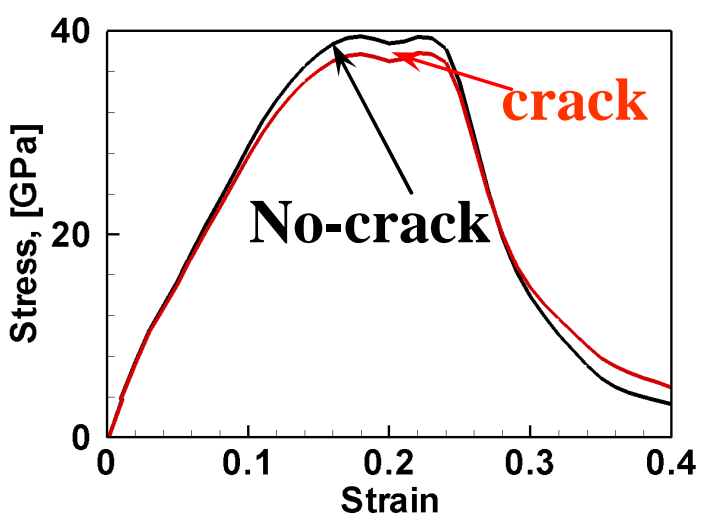

(a)

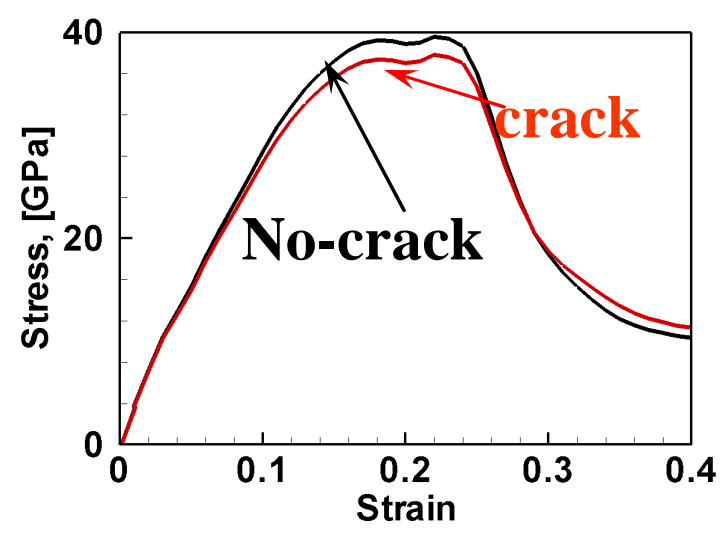

(b)

Figure 5 Virial stress in the loading direction Vs. uniaxial true strain plots for (a) microstructure $\mathrm{S} 2_{2}$ with and without initial crack and (b) for microstructure $\mathrm{S} 2_{4}$ with and without initial crack 


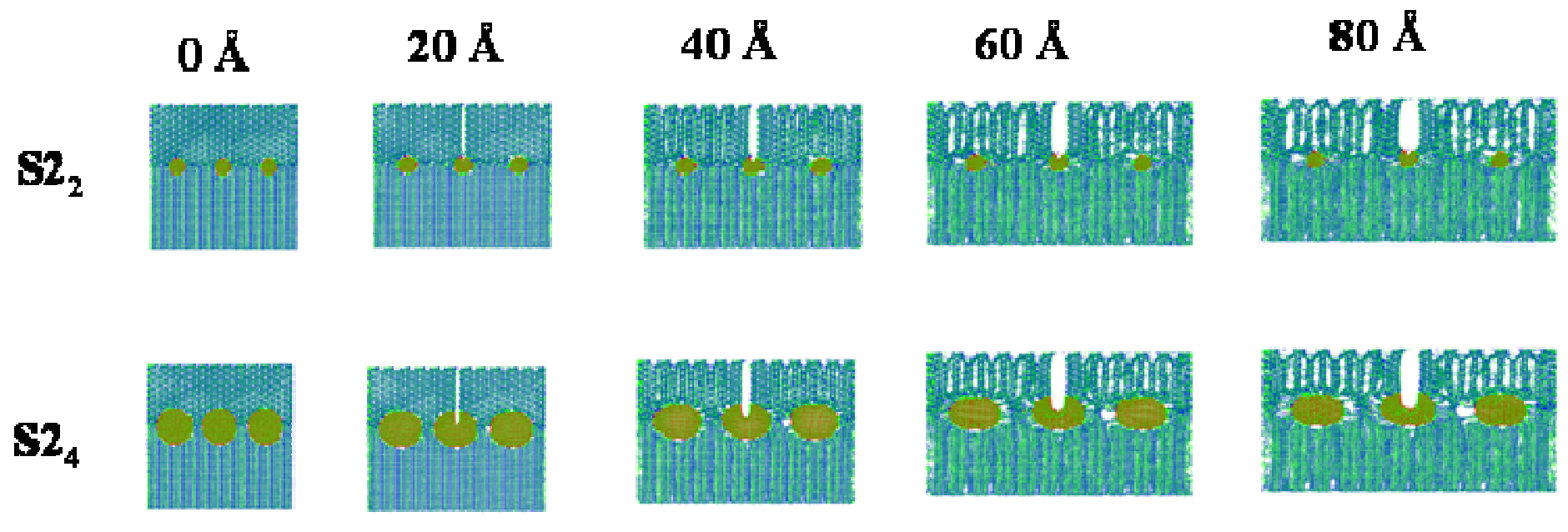

Figure 6 Viewgraphs showing various stages of deformation in $\mathrm{S} 2_{2}$, and $\mathrm{S} 2_{4}$ in the presence of initial crack. The displacement shown is the tensile deformation as a result of applied uniaxial strain rate on the nanocomposites 

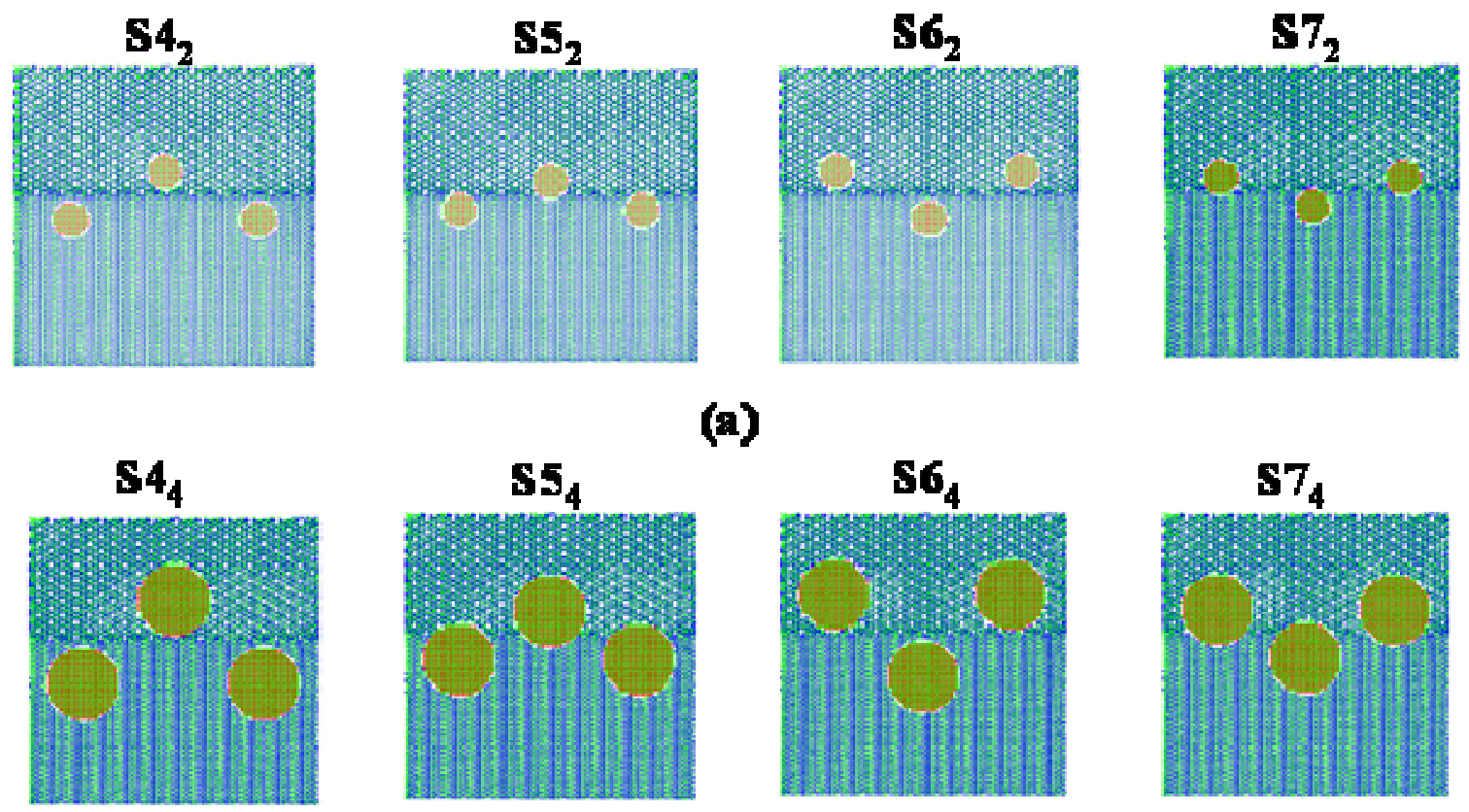

(b)

Figure 7 Second set of atomistic microstructures analyzed. The displacement shown is the tensile deformation as a result of applied uniaxial strain rate on the nanocomposites 


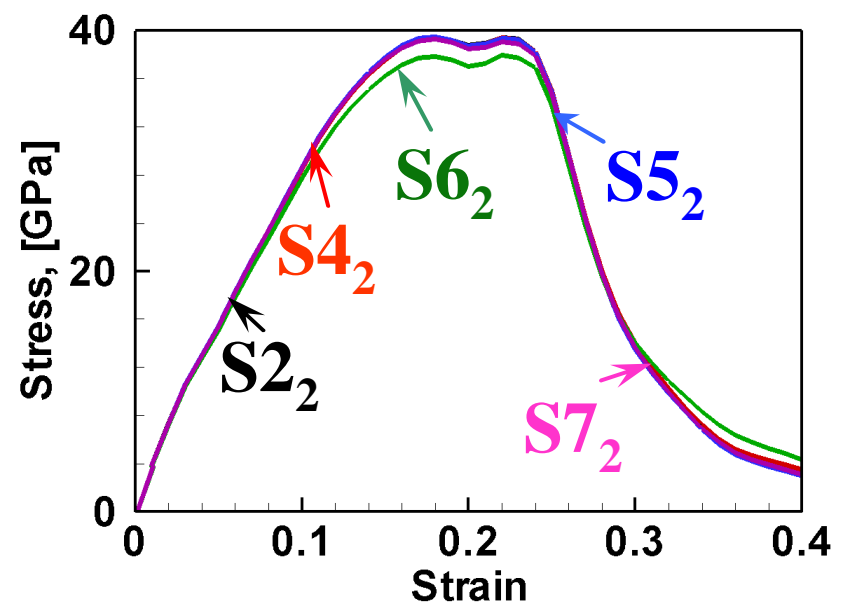

(a)

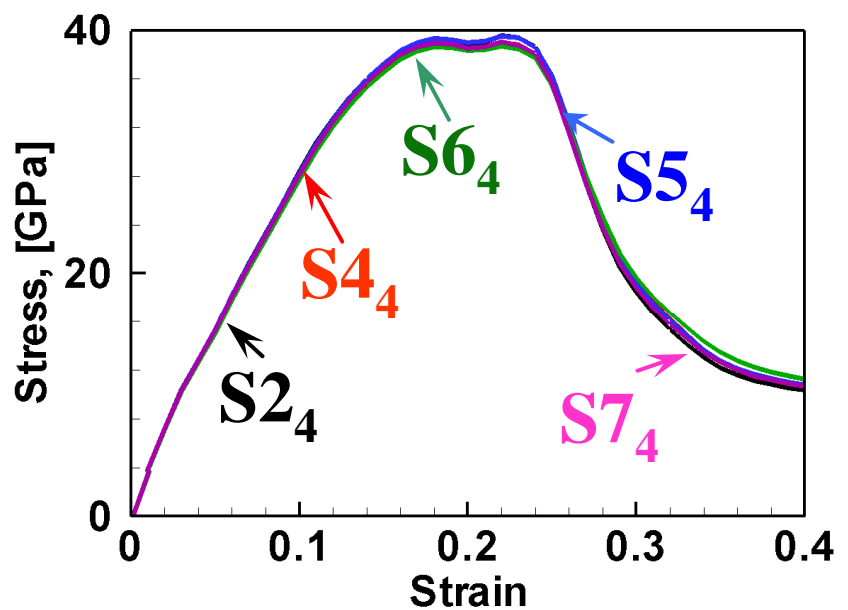

(b)

Figure 8 Virial stress in the loading direction Vs. uniaxial true strain plots for (a) microstructures $\mathrm{S}_{2}, \mathrm{~S}_{2}, \mathrm{~S}_{2}, \mathrm{~S}_{2}$, and $\mathrm{S} 7_{2}$ and (b) for microstructures $\mathrm{S} 2_{4}, \mathrm{~S}_{4}, \mathrm{~S}_{4}, \mathrm{~S}_{4}$, and $\mathrm{S}_{4}$ 

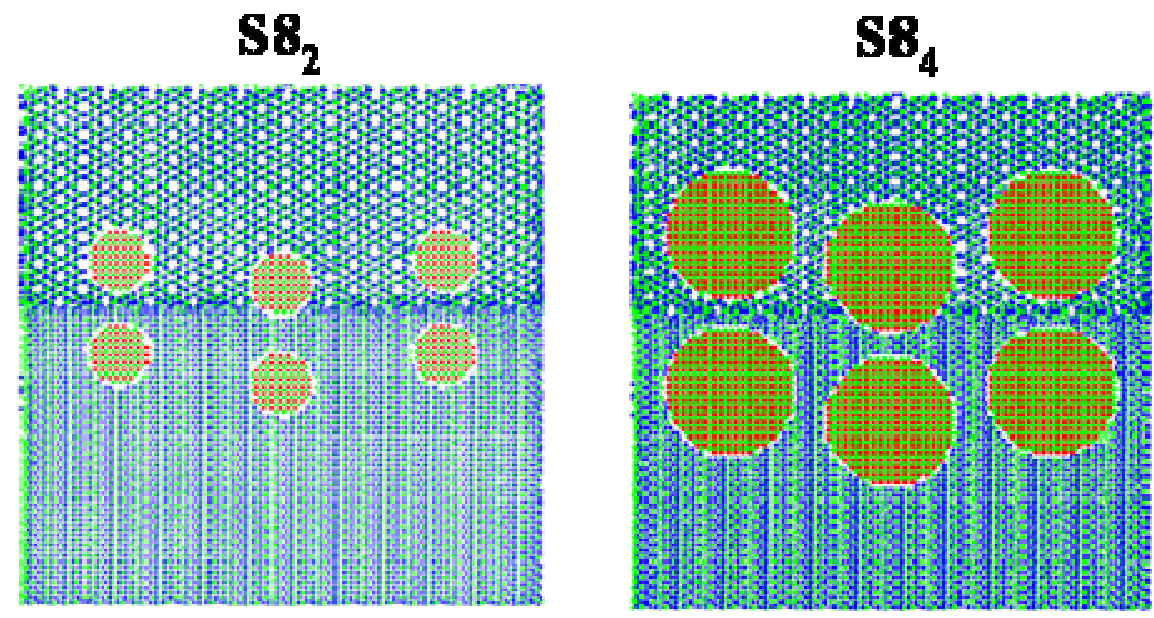

S9 ${ }_{2}$

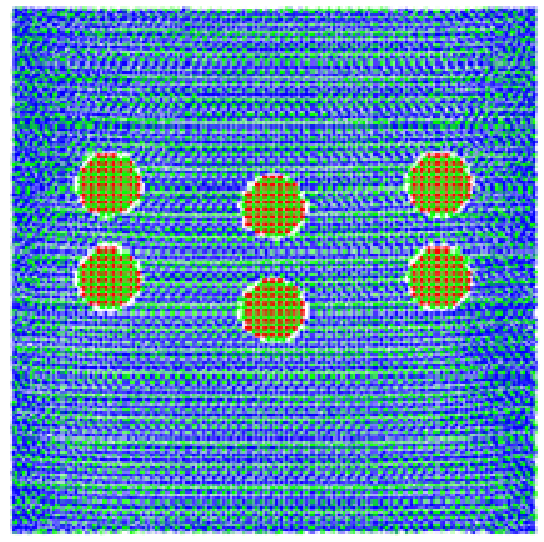

$\mathrm{Sg}_{4}$

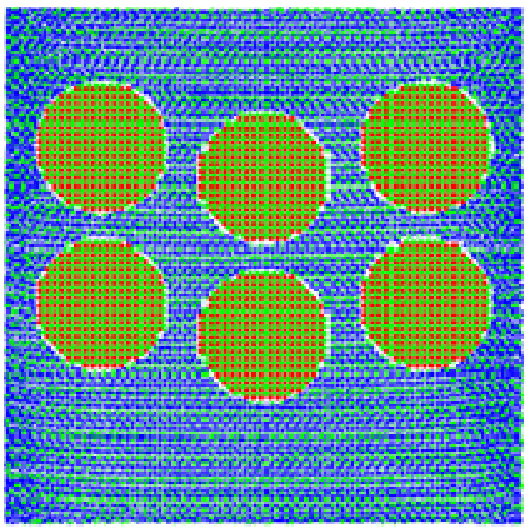

Figure 9 Third set of atomistic microstructures analyzed 


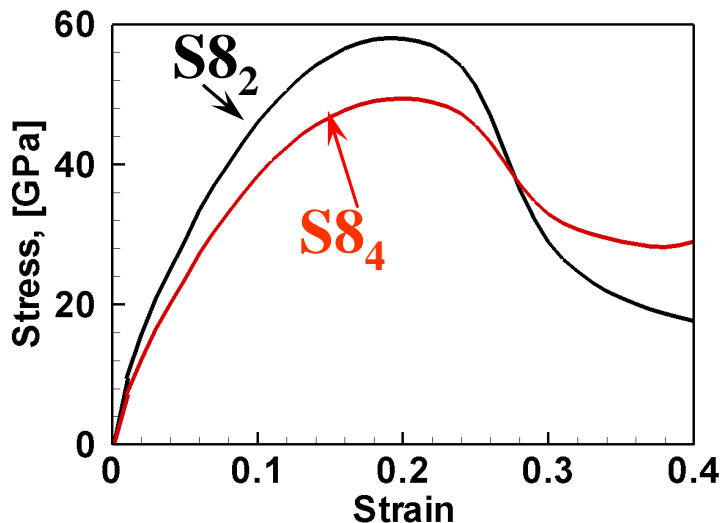

(a)

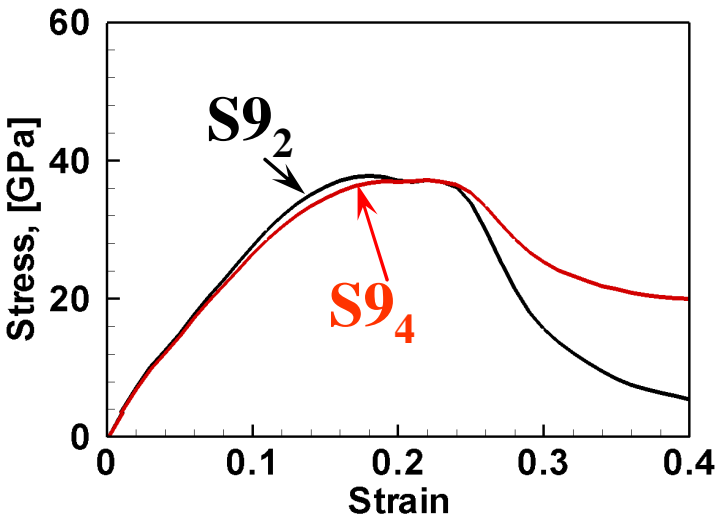

(b)

Figure 10 Virial stress in the loading direction Vs. uniaxial true strain plots for (a) microstructures $S 8_{2}$ and $S 8_{4}$ and (b) for microstructures $S 9_{2}$ and $S 9_{4}$ 


\section{A computer Aided Design Methodology to Design Ceramic}

\section{Nanocomposites ${ }^{3}$}

The need and the opportunity for significant savings in time and cost for engineered development of advanced nanomaterials coupled with tremendous growth in the past couple of decades in computational materials science has not yet been materialized into significant material design tool developments. Research applications involving design tool development for composite material design are at an early stage of development. The development of affordable processing technologies for composite materials is expected to considerably increase their demand in the near future. Of particular importance in the engineering design of composite materials for various applications is the ability to tailor the constituent materials and the internal architectures. This process is commonly known as composite materials tailoring. A wide variety of material combinations, reinforcement geometries and architectures to choose from represents a big challenge for materials developers interested in material tailoring. This process differs from the structural design of composites, which primarily pertains to decisions such as laminate stacking sequences, dimension of structural constituents, etc. Extended research has focused on the conventional design optimization of laminated composites for cost, weight, stiffness and strength. Haftka et al.[80] described how to find an efficient composite structural design that meets the stiffness and strength requirements for a certain application by not only sizing the cross-sectional areas and member thickness, but also by local or global tailoring of the material properties through selective use of orientation, number, and stacking sequence of laminate that

\footnotetext{
${ }^{3}$ A major part of this work has been published in International Journals with Citation "A. Mejia-Rodriguez, G., Renaud, J.E., and Tomar V., 2008, A variable fidelity model management framework for designing multiphase materials, ASME J. Mech. Design, vol. 130, 091702-1

B. Mejia-Rodriguez, G., Renaud, J.E., and Tomar V., 2010, Multiobjective composite material design using the variable fidelity model management optimization framework, in press in Engineering Optimization

C. Mejia-Rodriguez, G., Renaud, J.E., Kim, H., and Tomar V., 2010, Sequential approximate optimization based robust design of Sic-Si3N4 nanocomposite microstructures, Submitted June 15 2010"
} 
make up the composite laminate. Bruyneel ${ }^{[81]}$ presented a general and effective procedure based on a mathematical programming approach for composite structures optimal design, under weight, stiffness and strength criteria, in which it is shown that not only ply thickness but also fiber orientations should be considered as design variables. This investigation focuses on tailoring of microstructures of $\mathrm{SiC}_{-} \mathrm{Si}_{3} \mathrm{~N}_{4}$ nanocomposites using design optimization techniques.

Design optimization of nanocomposite morphologies has gained attention in recent few years. The inverse problem of determining an optimal microstructure for a desired application is a challenging task. The designer has to first specify the desired mechanical properties for the application, which may involve one or more properties, such as an increase in the strength, toughness, creep resistance, etc. Once the critical properties have been identified, then the task is to synthesize the microstructure that will yield the desired properties. This procedure has been traditionally accomplished by trial-and-error based approaches and depends considerably on the designer's intuition and experience. For this reason, obtaining new materials has been a time consuming and an expensive process. Accordingly, a systematic method capable of synthesizing the optimal microstructure that will satisfy the design requirements, while reducing cost and time, is desired. Such methodology will be of much practical value to the developers of the new generation of advanced materials. Ikeda[82] presented a methodology to design alloys using a genetic algorithm and molecular dynamics simulations. Kulkarni et al.[83] presented a methodology to tailor the microstructure of $\mathrm{Al}-\mathrm{Mg}-\mathrm{Sc}-\mathrm{Zr}$ alloys for developing "materials by design". Theoretical and phenomenological models were used to correlate the microstructure to macroscopic strength and ductility. A genetic algorithm was used to find the optimal microstructure for satisfying user-defined design requirements. Sadagopan and Pitchumani[84] presented a tailoring framework based on the combinatorial optimization technique of genetic 
algorithms in conjunction with a property model base consisting of analytical microstructureproperty relationships. The properties of interest were composite density, elastic modulus, thermal conductivity, and coefficient of thermal expansion. A difficult task in the work of Kulkarni et al.[83], and Sadagopan and Pitchumani[84] was figuring out the correlation between the microstructure and macroscopic properties. This lead to problems in optimizing the microstructure because of the large number of model variables and the lack of models based on first principles.

Most other researchers have addressed material design from a conceptual standpoint [85, 86]. Olson[85] introduced a systems based approach that integrates processing, structure, property, and performance relations for the conceptual design of multilevel-structured materials. Ashby[86] pointed out that in the material design process the objective requirements are often conflicting. The use of multi-objective optimization methods in a material design problem was demonstrated to handle the trade-off between competing objective functions. Later, Ashby and Bréchet[87] focused on combining two or more existing materials to create a hybrid, to allow a superposition of their properties in a material design framework. Seepersad et al.[88] presented a robust topology design method for designing materials on mesoscopic scales by topologically and parametrically tailoring them to achieve elastic properties that are superior to those of standard or heuristic designs, customized for large-scale applications, and less sensitive to imperfections in the material. McVeigh et al.[89] proposed a multi-resolution analysis framework for material design, in which a material is physically decomposed to each individual scale of interest, and the deformation and constitutive behavior of each scale can then be examined separately while determining the overall material properties. Pelegri and Tekkam[90] proposed a novel methodology for the maximization of mode I critical delamination fracture 
toughness using design of experiments and response surface, that allows continuous and discrete factors to be considered simultaneously like the width, length, thickness, stacking angle, and stacking sequence of the laminate. While all approaches mentioned above offer significant new developments, the use of such approaches is not amenable to time intensive calculations such as molecular simulations which almost always take days to complete to generate only one design point. Correspondingly, all the above approaches cannot be used to design complex multiphase materials using advanced time-intensive numerical tools for material behavior analyses such as the mesoscale finite element method (FEM) and the molecular dynamics (MD) method owing to the heavy computational cost of such tools.

A mesoscale FEM model takes days to complete one calculation[91]. The intensive computational cost of these simulations makes the use of iterative design and optimization procedures based on such tools prohibitively expensive to perform. One, therefore, requires a design approach that can incorporate multiple simulations (multi-physics) of varying fidelity such as FEM and MD in design iterations in an iterative manner, while simultaneously reducing the design cycle time. Recently, some efforts have been made in that direction by the authors. Mejía-Rodríguez et al.[92] presented a material design tool based on the variable fidelity model management framework. In the tool, complex "high fidelity" FEM analyses were performed only to guide the analytical "low-fidelity" model toward the optimal material design goal. The tool was applied to obtain the optimal distribution of a second phase, silicon carbide (SiC) fibers, in a silicon-nitride $\left(\mathrm{Si}_{3} \mathrm{~N}_{4}\right)$ matrix to obtain continuous fiber $\mathrm{SiC}-\mathrm{Si}_{3} \mathrm{~N}_{4}$ ceramic composites $(\mathrm{CFCCs})$ with optimal fracture toughness. Later[93], the material design tool was used to obtain the optimal microstructure of continuous fiber $\mathrm{SiC}_{-} \mathrm{Si}_{3} \mathrm{~N}_{4}$ ceramic composites (CFCCs) with optimal strength and creep resistance. In the tool, complex 3 dimensional (3-D) "high fidelity" FEM 
based analyses are performed only to guide the 2 dimensional "low-fidelity" model calculations toward the optimal material design goal. These investigations have shown how models with different input design variables could be handled and integrated efficiently by a trust region model management framework in application to the design of multiphase composite materials. The tool offered a significant development in material design optimization schemes where time consuming simulations could be performed efficiently to predict optimal microstructures. However, the tool in the presented works could not deal with the generation of realistic microstructural material morphologies and could not account for uncertainty quantification. This is the focus of the present investigation.

This work presents a systematic optimization methodology to predict optimal material microstructures, while considering uncertainties in the microstructural representations with simultaneous reduction in the design cycle time. The problem in hand is to predict the most suitable microstructural phase morphologies for desired deformation energy of $\mathrm{SiC}_{-} \mathrm{Si}_{3} \mathrm{~N}_{4}$ nanocomposites at $1500{ }^{\circ} \mathrm{C}$, and $1600{ }^{\circ} \mathrm{C}$. Statistical uncertainties inherent to computational microstructural generation (processing variations) are quantified, and used in the proposed methodology to predict robust nanocomposite microstructures with properties insensitive to a prespecified range of changes in microstructures. The focus is on finding robust microstructures that maximize the deformation energy at two temperatures: $1500{ }^{\circ} \mathrm{C}$ and $1600{ }^{\circ} \mathrm{C}$. FEA based tensile tests are performed on the $\mathrm{SiC}_{-} \mathrm{Si}_{3} \mathrm{~N}_{4}$ microstructures to extract their deformation energy, which is considered as the area under the force-displacement curve resulting from corresponding tensile tests. A sequential approximate optimization under uncertainty algorithm is applied to six different test problems. The first four cases obtain optimum microstructures at specific temperatures. In the last two cases, the focus is on obtaining bi-objective based optimum 
microstructures that will perform well at both temperatures. Although the material of interest in this investigation is $\mathrm{SiC}_{\mathrm{C}} \mathrm{Si}_{3} \mathrm{~N}_{4}$, the methodology is not restrictive and could be an invaluable design tool to support the development of new materials.

\section{Constitutive models}

Brittle materials, including ceramics such as $\mathrm{SiC}$ and $\mathrm{Si}_{3} \mathrm{~N}_{4}$, exhibit unique deformation and failure characteristics at high temperatures. A constitutive model in the form of Eq. (14) proposed by Lei et al.[94] for modeling $\mathrm{Si}_{3} \mathrm{~N}_{4}$ undergoing laser-assisted machining has been used to model the deformation and bulk properties of $\mathrm{Si}_{3} \mathrm{~N}_{4}, \mathrm{SiC}$, and an amorphous silicate based grain boundary (GB). In the model, the temperature effect on the flow stresses is accounted through the viscosity of $\mathrm{Si}_{3} \mathrm{~N}_{4}$ and $\mathrm{SiC}$ at elevated temperatures. The model allows to understand the effect of temperature and grain size on the material behavior. The constitutive model implemented isotropic hardening plasticity in user subroutine UMAT of popular FEA package ABAQUS. The Newton's forward formula was used to solve for the incremental plastic strain at each step and for every element which was found to have yielded,

$$
\sigma=\sigma_{o}\left[1+\left(\frac{\varepsilon}{\varepsilon_{0}}\right)^{n}\right]\left[\frac{\dot{\varepsilon}}{\dot{\varepsilon}_{0}} \cdot \exp \left(\frac{Q}{R T}\right)\right]^{m} .
$$

In Eq. 1 , for liquid-phase sintered $\beta-\mathrm{Si}_{3} \mathrm{~N}_{4}$ (SN88), $\sigma_{0}$ (reference stress) is estimated to be 28 $\mathrm{MPa}$ using the yield stress measured at strain rate, $\dot{\varepsilon}=2 \times 10^{-9} \mathrm{~s}^{-1}$ and temperature, $T=1400^{\circ} \mathrm{C}$ [95]. The reference strain $\left(\varepsilon_{0}\right)$, reference strain rate $\left(\dot{\varepsilon}_{0}\right)$, and the strain exponent $(n)$ are assumed to be $\varepsilon_{0}=0.3, \dot{\varepsilon}_{0}=1 \mathrm{~s}^{-1}$, and $n=0.34$, respectively, according to the strain hardening curve observed for silicon nitride[96]. $Q$ is the activation energy determined to be approximately $740 \mathrm{~kJ} / \mathrm{mol}$ from literature [97, 98]. $R$ is the universal gas constant $\left(8.31447 \mathrm{~J} /\left(\mathrm{mol} /{ }^{\circ} \mathrm{K}\right)\right)$. The value of the exponential coefficient, $m$, is determined to be 0.061 according to the constitutive model 
developed by Lei et al.[94] for $\mathrm{Si}_{3} \mathrm{~N}_{4}$ undergoing laser assisted machining (LAM). The conditions of LAM are used because the material is exposed to hard conditions (high strain rates and high temperatures). Lei et al.[94] have found that temperature has much stronger influence on the cutting forces and thus on the material strength than the effect caused by cutting speed under the conditions of LAM. In this research the value of $m$ is estimated to be 0.09 . Accordingly, the influence of temperature on the flow stress results in this quantity decreasing to around $700 \mathrm{MPa}$ and $400 \mathrm{MPa}$ at $1400{ }^{\circ} \mathrm{C}$ and $1600{ }^{\circ} \mathrm{C}$ respectively[99, 100].

For Hexology (sintered $\alpha-\mathrm{SiC}$ ), $\sigma_{0}$ (reference stress) is estimated to be $400 \mathrm{MPa}$ using the yield stress measured at $\dot{\varepsilon}=1 \times 10^{-9} \mathrm{~s}^{-1}$ and $T=1400^{\circ} \mathrm{C}[95,101]$. The activation energy $Q$ is determined to be approximately $980 \mathrm{~kJ} / \mathrm{mol}$ from literature[95, 101]. The reference strain, strain rate, the strain exponent and the value of $m$ are assumed to be $\varepsilon_{0}=0.17, \dot{\varepsilon}_{0}=1 \mathrm{~s}^{-1}, n=0.5$, and $m=-$ 0.001 respectively. Accordingly, the influence of temperature on the flow stress results in this quantity decreasing to around 445 and $447 \mathrm{MPa}$ at $1400{ }^{\circ} \mathrm{C}$ and $1600{ }^{\circ} \mathrm{C}$ respectively[102].

For the amorphous GB, a limited experimental data is found in the literature. Since GBs in $\mathrm{Si}_{3} \mathrm{~N}_{4}$ are responsible for the increase in fracture resistance, it was assumed that GBs have reduced strength and higher fracture energy in comparison to the $\mathrm{Si}_{3} \mathrm{~N}_{4}$ phase. Such assumption

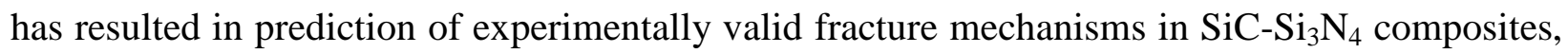
Tomar[103-105]. The following parameters were assumed for GBs: $\sigma_{0}$ (reference stress) is estimated to be $28 \mathrm{MPa}$ using the yield stress of $\dot{\varepsilon}=2 \times 10^{-9} \mathrm{~s}^{-1}$ at $T=1400^{\circ} \mathrm{C}$, and with $Q=733$ $\mathrm{kJ} / \mathrm{mol}$. The reference strain, strain rate, the strain exponent and the value of $m$ are assumed to be $\varepsilon_{0}=0.3, \dot{\varepsilon}_{0}=1 \mathrm{~s}^{-1}, n=0.34$, and $m=0.09$, respectively. Figure 1 shows the stress-strain curve for $\mathrm{Si}_{3} \mathrm{~N}_{4}, \mathrm{SiC}$ and GB as a function of temperature. The materials models described above for $\mathrm{Si}_{3} \mathrm{~N}_{4}$, $\mathrm{SiC}$ and GB are good at describing their stress-strain behavior. 

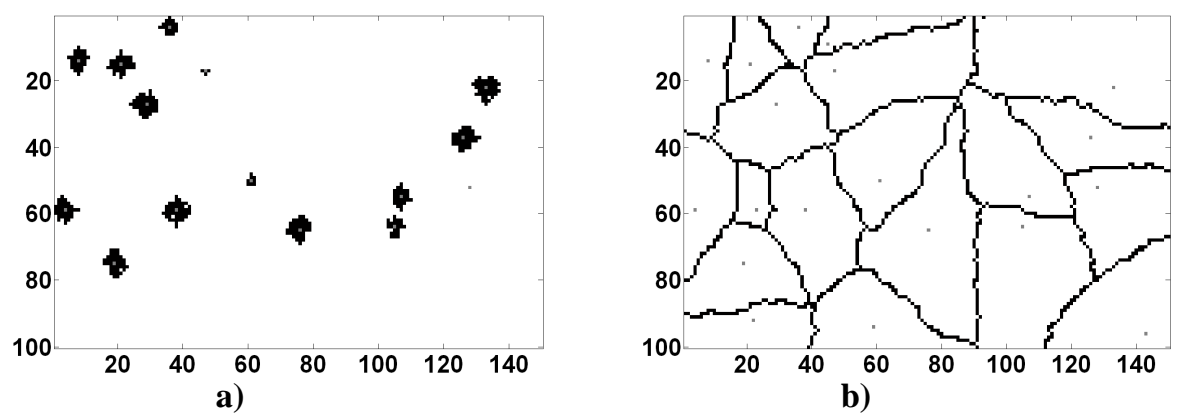

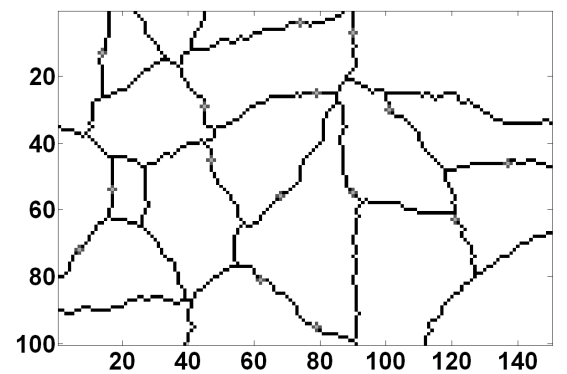

c)

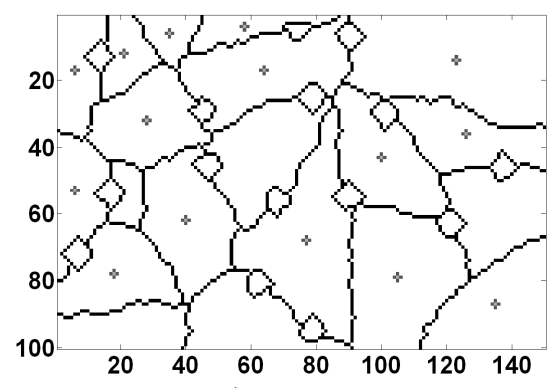

e)

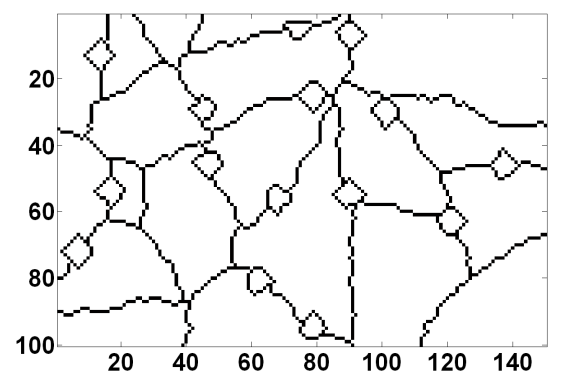

d)

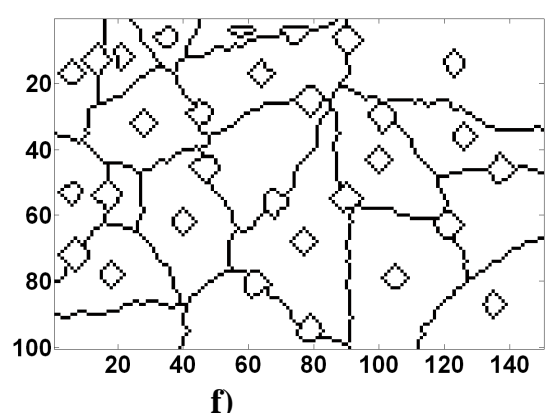

f)

Figure 2. Schematic showing an example of the nucleation and growth mechanisms for the $\mathrm{SiC}-\mathrm{Si}_{3} \mathrm{~N}_{4}$ nanocomposites: (a) Nucleation and growth for $\mathrm{Si}_{3} \mathrm{~N}_{4}$ grains at iteration 15 , (b) final $\mathrm{Si}_{3} \mathrm{~N}_{4}$ microstructure, (c) intergranular $\mathrm{SiC}$ grains start growing after being nucleated randomly along the $\mathrm{GB}$, (d) final $\mathrm{Si}_{3} \mathrm{~N}_{4}-\mathrm{SiC}$ intergranular microstructure, (e) intragranular $\mathrm{SiC}$ grains start growing after being nucleated randomly inside the $\mathrm{Si}_{3} \mathrm{~N}_{4}$ grains, and (f) the final $\mathrm{Si}_{3} \mathrm{~N}_{4}-\mathrm{SiC}$ nanocomposite microstructure. 


\section{Microstructural Simulation Techniques}

In structure-property correlation based simulations, microstructures are characterized by the crystalline orientation, grain sizes, second phase dispersion, and GBs. For material design optimization it is desired that microstructure evolution algorithms are also embedded in the design optimization codes. This will result in on the fly changes in the microstructures corresponding to the need of the optimization algorithms. The Johnson-Mehl model[106-108] and the tessellation process for a Voronoi network[108-110] are the most well known techniques that have been used to develop microstructure evolution for realistic microstructural representation.

Microstructure generation simulations begin with the generation of a set of random points. These points or nodes serve as the nucleation points of the grain structure. Once these points are defined, a network is generated around them that may be a function of geometry (Voronoi network) or may be a function of grain growth at a constant rate (Johnson-Mehl). The method used in this work uses a lattice model proposed by Choi et al.[111]. The polycrystalline microstructure evolution mechanism is described by nucleation and growth mechanisms. Since the nucleation and growth mechanism well explain the polycrystalline microstructure evolution, most of the microstructure evolution simulation techniques use this mechanism. Frost et al.[107] investigated the effect of nucleation conditions on the topology of grain structure

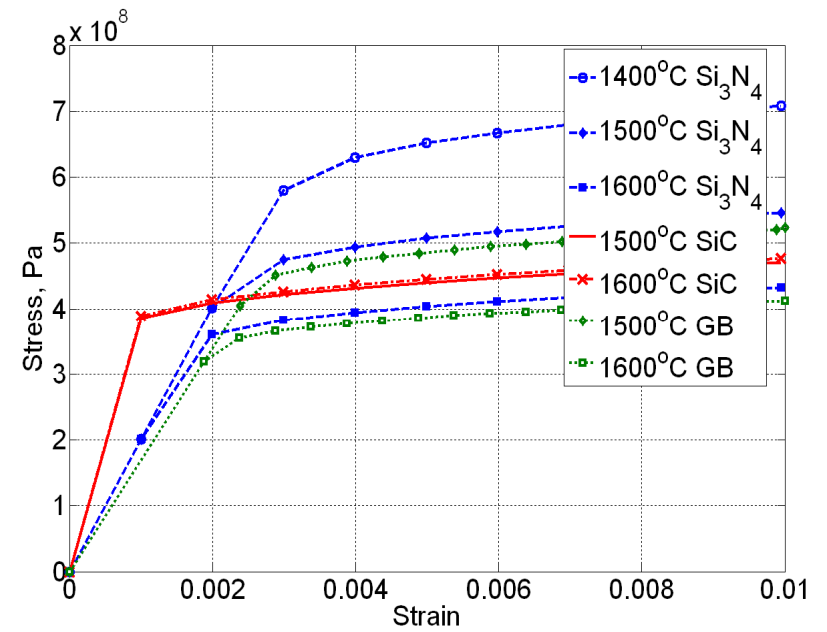

Figure 1. Stress-Strain curve for monolithic samples with $\mathrm{Si}_{3} \mathrm{~N}_{4}, \mathrm{SiC}$ and $\mathrm{GB}$ properties as a function of temperature. 
using the model used in this research. These conditions include simultaneous nucleation (all nucleation takes place at the same time), continuous nucleation (constant nucleation rate), and intermediate nucleation in which the nucleation rate declines with time. For their model, nucleation and growth continue until the unit area is completely filled. For analysis of grains size distribution (GSD), the probability density function (PDF) of following gamma distribution is used[112],

$$
P\left(A^{\prime}\right)=\frac{\beta^{\beta}}{\Gamma(\beta)} A^{B^{B-1}} e^{-B A^{\prime}}
$$

In Eq. (15) $A^{\prime}$ is the normalized grain size, $\Gamma(\beta)$ is the gamma function. The parameter $\beta$ is the key parameter of GSD. It characterizes the shape of the distribution and has the range of 1.0 3.65. The value of $\beta=3.65$ corresponds to the simultaneous nucleation and $\beta=1$ corresponds to the continuous nucleation[112, 113]. Since this research focuses on the two phase polycrystalline $\mathrm{SiC}-\mathrm{Si}_{3} \mathrm{~N}_{4}$ as a target material, the method presented by Choi et al.[111] is extended in this investigation to handle two phase composite materials. The nucleation and growth procedures are briefly described as follows:

First $\mathrm{Si}_{3} \mathrm{~N}_{4}$ grains are grown in the following steps: (i) the nucleation sites are randomly picked for each time interval, and (ii) while the nucleated sites grow with a certain probability, new nucleation takes place. Steps (i) and (ii) are repeated for every time interval (step) until the lattice domain is completely filled with the desired number of $\mathrm{Si}_{3} \mathrm{~N}_{4}$ grains. In this process, the number of nucleation sites is directly controlled at each time step. Once $\mathrm{Si}_{3} \mathrm{~N}_{4}$ grains have formed according to the desired grain size distribution, the $\mathrm{SiC}$ grains are grown in the $\mathrm{Si}_{3} \mathrm{~N}_{4}$ microstructure in the following steps: (i) the intergranular SiC nucleation sites are randomly picked on the $\mathrm{Si}_{3} \mathrm{~N}_{4}$ grain boundary, (ii) the intergranular nucleated sites are grown for a desired 
time, (iii) the intragranular $\mathrm{SiC}$ nucleation sites are randomly picked inside the $\mathrm{Si}_{3} \mathrm{~N}_{4}$ grains, and (iv) the intragranular nucleated sites are grown for a desired time.

Notice that the microstructure evolution mechanism is a stochastic process. For this reason, robust design methodologies have to be used in the design task to find the most suitable microstructures for desired applications. Figure 2 shows the nucleation and growth mechanisms for a SiC-Si ${ }_{3} \mathrm{~N}_{4}$ nanocomposite with $20 \mathrm{Si}_{3} \mathrm{~N}_{4}$ grains, and corresponding initial nucleation of 10 grains and nucleation rate of 1 . The number of intergranular $\mathrm{SiC}$ grains is 15 with a maximum growth of 5 cycles, and the number of intragranular SiC grains is 15 with a maximum growth of 4 cycles.

\section{Robust Optimization}

Robust Design Method, also called Taguchi Method, was originally developed by Genichi Taguchi. In 1980, Taguchi's introduction of the method to several American industries resulted in significant quality improvement in product and manufacturing process design. By consciously considering the noise factors (environmental variation during the product's usage, manufacturing variation, and component deterioration) and the cost of failure in the field the Robust Design method helps ensure customer satisfaction[114]. Taguchi introduced the concept of parameter design to improve the quality of a product whose manufacturing process involves significantly variability. Taguchi's parameter design reduces variation in performance by reducing the sensitivity of an engineering design to sources of variation rather than controlling the sources. Taguchi techniques were based on direct experimentation[115]. Of more interest is Taguchi's definition of the "goodness" of a design. Whereas various other approaches assume that a good design meets a set of well-defined functional, technical performance, and cost goals, Taguchi 
states that a good design minimizes the quality loss over the life of the design, where quality loss is defined to be the deviation from the desired performance[116].

In robust or statistical optimization, one is interested not only in parameter sensitivities but also in finding the feasible combination of design variables that minimize both objective function and its sensitivity to design variable variation[115]. Robust optimization significantly increases the computational requirements in comparison to traditional optimization, and the computational cost further increases by using a conventional gradient based optimization method, due to the invoking of finite difference calculations. In this investigation, response surface approximations of the objective function and its variation are successively created and incorporated in a surrogate-based optimization method under uncertainty, where uncertainty is quantified through the use of experiments.

\section{Experimental Design-Based Robust Optimization}

The robust optimization strategy followed in this work is based on the method presented by Su and Renaud[115], where Taguchi's orthogonal arrays are used to specify an experimental design plan for evaluating the variation of the objective function and constraints. In robust design optimization (RDO) there is a trade off between reducing the variation of the function and optimizing the function value itself. Robust optima are less sensitive to the variation of the design variables, but the function value tends to be larger than that obtained from conventional optimization (i.e. a small decrease in the performance is traded-off for a decrease in the performance variation). In the light of this recognition, robust optimization can be considered as a multi-objective optimization, where the conventional objective function and its variation are the objectives under consideration[117, 118].

The optimization formulation in this investigation takes the form: 


$$
\begin{gathered}
\text { Minimize } \quad F(\mathrm{x})=\alpha \cdot \bar{f}_{p}(x)+(1-\alpha) \cdot \operatorname{vrt}\left[f_{p}(x)\right] \\
\text { Subject to: } \quad x^{L} \leq x \leq x^{U}
\end{gathered}
$$

where

$$
\begin{gathered}
f_{p}(x)=f(x)+\Omega[R, g(x)] \\
v r t\left[f_{p}(x)\right]=\sqrt{\frac{1}{n s} \sum_{i=1}^{n s}\left(f_{p_{i}}-\bar{f}_{p}\right)^{2}} \\
\Omega=R[\hat{g}(x)]^{2} \\
\hat{g}(x)=\left(\begin{array}{cc}
g(x) & \text { if } g(x)<0.0 \\
0 & \text { if } g(x) \geq 0.0
\end{array}\right) \\
g_{j}(x) \geq 0 \quad j=1, \ldots, J
\end{gathered}
$$

The robust objective function has two parts, the function and its variation. The variation vrt $\left[f_{p}(\mathrm{x})\right]$ is defined as the root-mean square value of the difference between the value of the sample points and the mean $\bar{f}_{p}(x)$ of the values at all sample points. Note that there are no explicit constraints used in this formulation. Constraints are considered as penalty terms. A penalty function formulation, Eq. (18), is adopted in this method to avoid the undesired overhead associated with robust constraint gradient calls. The parameter $\alpha(0 \leq \alpha \leq 1)$ serves as a weighting factor in this bi-objective optimization. The larger the value of $\alpha$, the more significance the decision-maker puts on the conventional objective over its variation and vice versa. In this work values of $\alpha=1$ and $\alpha=0.5$ are investigated. In the work by Su and Renaud[115] the sample designs $i$ in Eq. (19) are selected by using an orthogonal array to determine the combination of design variables for fractional factorial experiments. In Su and Renaud[115], the objective functions are deterministic, and $v r t\left[f_{p}(\mathrm{x})\right]$ represents the variation in the deterministic function $f_{p}$ locally around the current design $x_{n}$, due to variation in $x$, see Fig. $3 a$. 


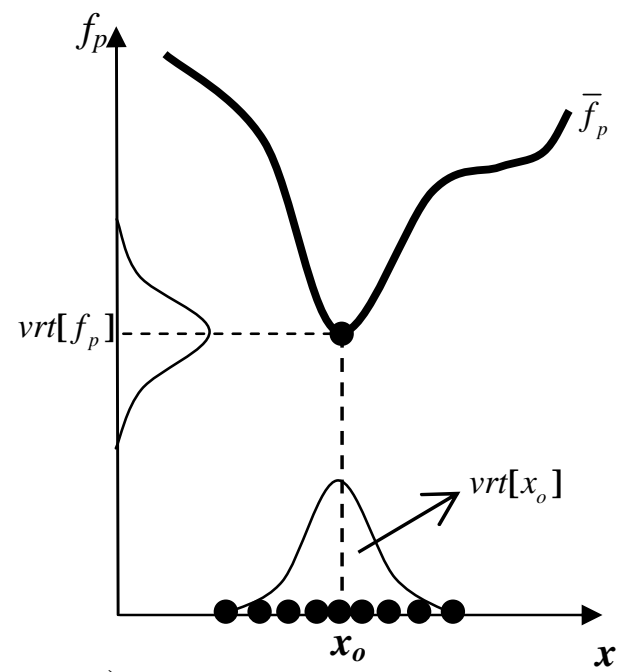

a)

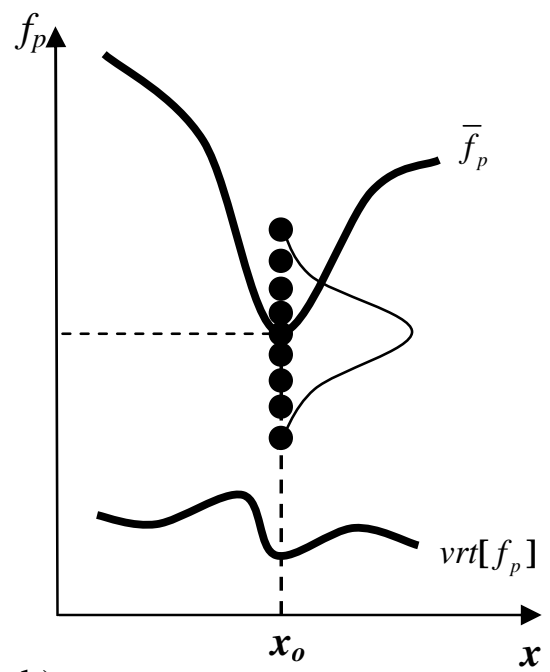

b)

Figure 3. Schematic showing differences between the type of functions used in the work of Su and Renaud[115], and this investigation: (a) Deterministic function with uncertainty in design variables, (b) Non-deterministic function with no uncertainty in design variables.

\section{Robust Design on a Non-Deterministic Function}

In this investigation the function (deformation energy) is a non-deterministic function, this is because of the characteristic of the random errors that arise in the microstructural generation simulations. Remember that the microstructure evolution mechanism is a stochastic process. For same input parameters (design variables) microstructures with different attributes are obtained, and as a result different responses (deformation energy values) are obtained. Uncertainty is quantified by repeating the same analysis 20 times at a given design point. This investigation does not consider variation in the design variables $x$, see Fig. $3 b$.

Thus, uncertainty is quantified to only obtain the mean and standard deviation parameters of the statistical data. The value of 20 was considered to be a good number to use, after performing a study on how the mean and standard deviation behaved as the number of simulations increased. 
Approximately at the $20^{\text {th }}$ simulation, an almost steady behavior in the mean and standard deviation of the simulations was observed.

\section{Surrogate Based-Design Optimization Under Uncertainty}

To perform optimization under uncertainty, surrogates of statistical data generated from a set of uncertainty quantification (UQ) analysis are integrated in a sequential approximate optimization (SAO) based algorithm, subject to move limits. SAO is a class of methods for the design of systems which are cost prohibitive to compute large numbers of function evaluations. In typical SAO methods a local response surface approximation (RSA) is built from a sampling of the design space about the current design. Once the RSA has been constructed it is very inexpensive to evaluate and its optimal point can be found using traditional constrained nonlinear optimizers. The optimal point of the RSA is compared to the true model. If the design has improved, the process repeats, sequentially building approximations, until the method converges. There are extensive examples of SAO methods in the literature, including the work of Rodriguez et al.[119, 120], Perez et al.[121, 122], and Rasmussen[123]. The optimization algorithm used in this investigation is shown in Fig. 4. 


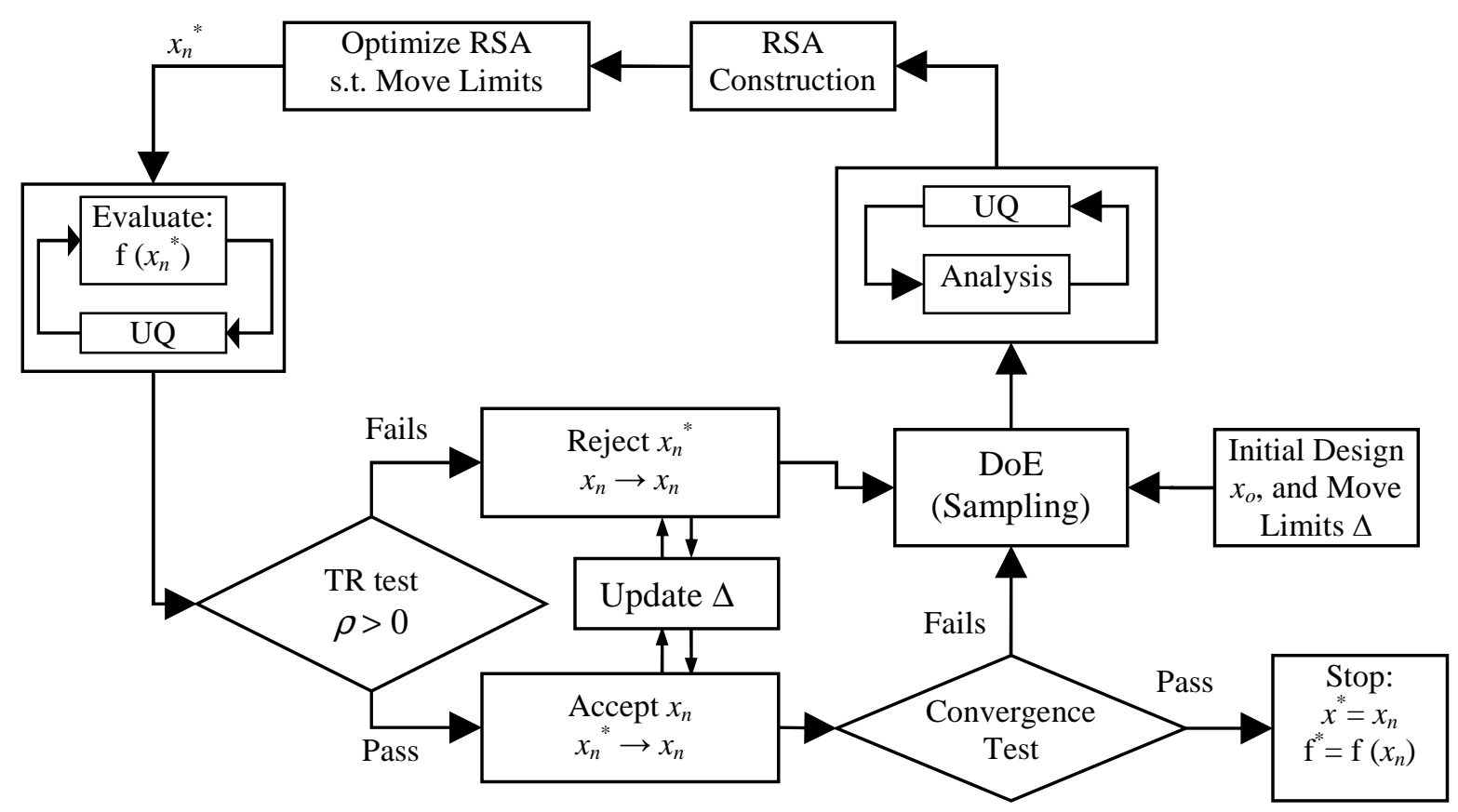

Figure 4. A schematic flowchart showing the sequential approximate optimization under uncertainty algorithm.

Important components of the optimization algorithm are the trust region (TR) test and the convergence test, which are described next.

Trust Region (TR) Management: In order to help guarantee convergence of the SAO framework, a trust region model management strategy is employed. This method provides a means for adaptively managing the allowable move limits for the approximate design space. A trust region ratio allows the trust region model management framework to monitor how well the approximation matches the true objective function. The trust region ratio, $\rho_{n}$, is calculated at the new candidate point $x_{n}{ }^{*}$, as

$$
\rho_{n}=\frac{f_{p}\left(x_{n}\right)-f_{p}\left(x_{n}^{*}\right)}{\tilde{f}_{p}\left(x_{n}\right)-\tilde{f}_{p}\left(x_{n}^{*}\right)}
$$


In Eq. (23), $f_{p}$ and $\tilde{f}_{p}()$ are the functions for the true and approximate models, and $x_{n}$ is the current design. The trust region size is governed by the following standard rules

$$
\Delta_{n+1}=\left\{\begin{array}{cc}
0.25 \Delta_{n}: & \rho_{n} \leq 0.25 \\
\Delta_{n}: & 0.25<\rho_{n}<0.75 \\
2 \Delta_{n}: & 0.75 \leq \rho_{n}
\end{array}\right.
$$

Physically, $\rho$ represents how good the approximation represents the true objective function. If $\rho$ is near 1 , the approximation is quite good. If $\rho$ is near zero, then the approximation is not as good, but it still captures the minimization trend. If $\rho$ is negative, then the point is a worse design, the point is rejected and the trust region size is reduced.

Convergence Test: Convergence can be determined by the following stopping criterion

$$
\begin{gathered}
\frac{\tilde{f}_{p}\left(x_{n}\right)-f_{p}\left(x_{n}\right)}{f_{p}\left(x_{n}\right)+1}<\varepsilon_{f 1} \\
\frac{f_{p}\left(x_{n}\right)-f_{p}\left(x_{n-1}\right)}{f_{p}\left(x_{n-1}\right)+1}<\varepsilon_{f 2} \\
\frac{\left\|x_{n}-x_{n-1}\right\|_{\infty}}{\left\|x_{n-1}\right\|_{\infty}+1}<\varepsilon_{x}
\end{gathered}
$$

In Eqns. (25)-(27), $\varepsilon_{f 1}, \varepsilon_{f 2}$ and $\varepsilon_{x}$ are tolerances supplied by the user, and $n$ is the current iteration counter. If any of the three inequalities at the current point are true, the algorithm is considered converged.

An important approach is followed to reduce the computational time associated with UQ during the initial iterations of the sequential approximate optimization under uncertainty algorithm. This approach consists in performing UQ, once the TR test fails $(-\rho)$ for the first time. Before the TR test fails $(-\rho)$, single evaluations of the performance function $(S)$ are performed at each design to construct the RSA. As a result of not passing the TR test, the candidate design 
$\left(x_{n}{ }^{*}\right)$ given by the optimizer is rejected, and the current design $\left(x_{n}\right)$ is revaluated using UQ, and all designs in coming iterations are evaluated using UQ until convergence is achieved in the algorithm.

\section{Test Problem}

For illustration purposes, Fig. 5 shows a sample finite element meshed microstructure. The size of the microstructure is $150 \mu \mathrm{m} \times 100 \mu \mathrm{m}$. The design variables used to control the microstructural phase morphologies are the number of Silicon Nitride grains $\left(n \_S i_{3} N_{4}\right)$, the number of intergranular Silicon Carbide grains (n_SiC_inter), the number of intragranular Silicon Carbide grains ( $n \_S i C \_$intra), the initial nucleation sites to generate the Silicon Nitride grains $\left(i n \_n \_S i_{3} N_{4}\right)$, the nucleation rate to generate the Silicon Nitride grains $\left(n u c_{-} r_{-} S i_{3} N_{4}\right)$, the time of growth of the intergranular Silicon Carbide grains $($ t_SiC_inter $)$, and the time of growth of the intragranular Silicon Carbide grains $\left(t \_S i C \_i n t r a\right)$. Other microstructural attributes can be extracted from all composites, such as the volume fraction and the grain size for each of the phases, and the grain size distribution for Silicon Nitride. A characteristic of the present problem is that all design variables can only receive integer values, therefore it can be considered as an integer programming problem. 


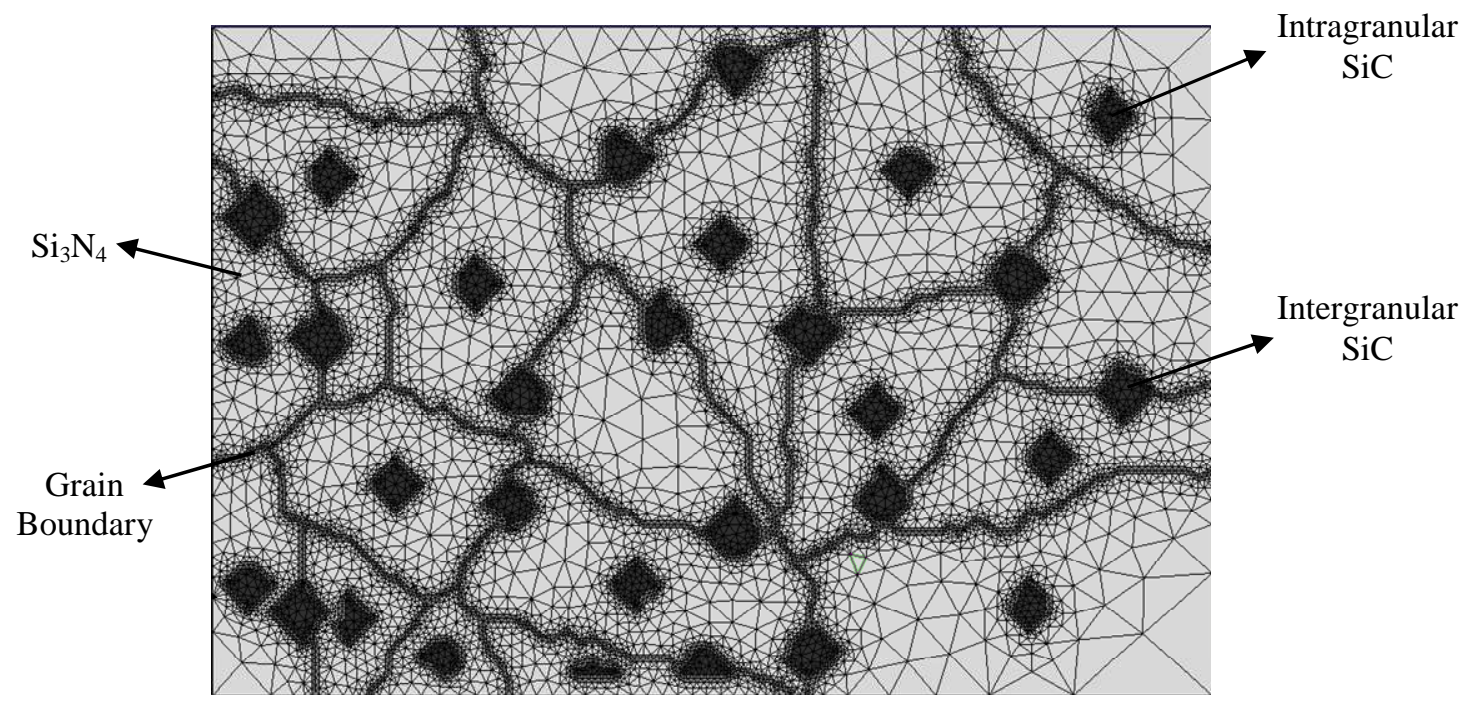

Figure 5. A sample finite element meshed microstructure.

The design variables used to generate the microstructure shown in Fig. 5 are $n_{-} S i_{3} N_{4}=20$, $n \_S i C \_$inter $=15, \quad n \_S i C \_i n t r a=15, \quad$ in $\_n \_S i_{3} N_{4}=10, \quad n u c \_r \_S i_{3} N_{4}=1, \quad t \_S i C \_i n t e r=5, \quad$ and t_SiC_intra $=4$. Regarding the other microstructural characteristics, the volume fraction of the grain boundary is $9.3 \%$, of $\mathrm{Si}_{3} \mathrm{~N}_{4}$ is $85.4 \%$, of the intergranular $\mathrm{SiC}$ is $3.2 \%$ and of the intragranular $\mathrm{SiC}$ is $2.1 \%$. The grain size of $\mathrm{Si}_{3} \mathrm{~N}_{4}$ is $15960 \mu \mathrm{m}^{2}$, of the intergranular $\mathrm{SiC}$ is $796 \mu \mathrm{m}^{2}$ and of the intragranular $\mathrm{SiC}$ is $517 \mu \mathrm{m}^{2}$. The grain size distribution of $\mathrm{Si}_{3} \mathrm{~N}_{4}$ is $\beta=2.9$.

The goal of this investigation is to find the most robust microstructures that maximize the deformation energy " $S$ " at $1500{ }^{\circ} \mathrm{C}$, at $1600{ }^{\circ} \mathrm{C}$, and at the equal weighted sum of both temperatures, subject to some design variable bounds and microstructural generation constraints. The optimization problem in the sequential approximate optimization under uncertainty algorithm can be stated as follows:

Minimize $S_{R}=\alpha \cdot\left[-\bar{S}_{r}\right]+(1-\alpha) \cdot \operatorname{vrt}\left[S_{r}\right]$

subject to: 


$$
\begin{aligned}
& 10 \leq n_{-} \mathrm{Si}_{3} \mathrm{~N}_{4} \leq 40 \\
& 1 \leq n_{-} \text {SiC_inter } \leq 25 \\
& 1 \leq n_{-} S_{S i C} \text { intra } \leq n_{-} S_{3} i_{3} \\
& 1 \leq \text { in_n_ } \text { Si }_{3} N_{4} \leq n_{-} \mathrm{Si}_{3} \mathrm{~N}_{4} \\
& 1 \leq n u c_{-} r_{-} S_{3} N_{4} \leq 10 \\
& 1 \leq t_{-} \text {SiC_inter } \leq 8 \\
& 1 \leq t_{-} \text {SiC_intra } \leq 8 \\
& \text { in } n_{-} \mathrm{Si}_{3} \mathrm{~N}_{4}+n u c_{-} r_{-} \mathrm{Si}_{3} \mathrm{~N}_{4} \leq n_{-} \mathrm{Si}_{3} \mathrm{~N}_{4}
\end{aligned}
$$

In Eq. (28), $S_{R}$ represents a response surface approximation of the objective function $S_{f}, \bar{S}_{r}$ represents the response surface approximation of the mean deformation energy $\bar{S}$ at design points, while $\operatorname{vrt}\left[S_{r}\right]$ is the response surface approximation of the standard deviation of the deformation energy $\operatorname{std}(S)$ at design points. The optimization process is carried out using the sequential quadratic programming (SQP) optimizer from the Mathworks Matlab's fmincon function, which is provided in the Optimization Toolbox.

FEA based tensile tests are performed on the $\mathrm{SiC}_{-} \mathrm{Si}_{3} \mathrm{~N}_{4}$ microstructures to extract their deformation energy. In this work, the deformation energy of the composite is considered as the area under the force-displacement curve resulting from corresponding tensile tests. The strength models presented in section II are assigned to the corresponding material in the nanocomposites. The way silicon nitride, silicon carbide and the grain boundary interact in the composite is by assuming that they are perfectly bonded, using a tie constraint.

Uncertainties are introduced in the $\mathrm{FE}$ analysis from the microstructural generation simulations. For the same input design variables, different microstructural characteristics are achieved, and as a result different deformation energy responses are obtained. For this reason, the sequential approximate optimization under uncertainty algorithm shown in Fig. 4, is used in six test problems to find the robust microstructures that maximize the deformation energy at two temperatures: $1500{ }^{\circ} \mathrm{C}$ and $1600{ }^{\circ} \mathrm{C}$. The first four cases use these temperatures separately to 
obtain optimum microstructures at specific temperatures. In the last two cases, both temperatures are used simultaneously to obtain multiobjective based optimum microstructures that will perform well at both temperatures.

\section{Results}

In the present investigation, values of $\alpha=1$ and $\alpha=0.5$ are used for the temperatures of 1500 ${ }^{\circ} \mathrm{C}, 1600{ }^{\circ} \mathrm{C}$, and for the bi-objective problem where both temperatures are equally weighted, for a total of six cases. In all cases, the initial trust region size (move limit) $\Delta$ at each dimension (design variable) equals $20 \%$ the design space size of the corresponding dimension. The fractional factorial design ( $1 / 2$ fraction) method is used to generate the design of experiments arrays needed in the algorithm. The number of design points generated at each iteration is not fixed. It depends on the number of samples needed to fill the design space, while satisfying the constraints presented in Eq. (28). Note that repeated samples that were already evaluated at previous iterations are not evaluated anymore, their value is taken from a database were all past designs and corresponding function values are stored.

It is important to remember the approach followed to reduce the computational time associated with UQ during the initial iterations of the sequential approximate optimization under uncertainty algorithm. The approach consists in performing UQ(calculate mean and standard deviation), once the TR test fails $(-\rho)$ for the first time. Before the TR test fails $(-\rho)$, single evaluations of deformation energy $(S)$ are performed at each design to generate the RSA.

Next, six test cases are presented. For cases A-D the convergence tolerance value for the design variables is $\varepsilon_{x}=0.1$ (see Eq. (27)), and the convergence tolerances for the function values are $\varepsilon_{f 1}=5.0 \times 10^{-6}$ and $\varepsilon_{f 2}=1.0 \times 10^{-5}$ (see Eqns.(25)-(26)). For cases E-F the convergence tolerance value for the design variables is $\varepsilon_{x}=0.1$, and the convergence tolerances for the function values 
are $\varepsilon_{f 1}=1.0 \times 10^{-3}$ and $\varepsilon_{f 2}=1.0 \times 10^{-3}$. To evaluate $\varepsilon_{x}$, each design variable is scaled to have a value between -1 and 1 .

\section{A. Temperature $=1500{ }^{\circ} \mathrm{C}$ and $\alpha=1$}

In this case, the initial design is $x_{o}=[20,10,10,4,4,5,5]\left(n \_S i_{3} N_{4}=20, n \_S i C \_i n t e r=10\right.$, $n \_S i C \_i n t r a=10, i n \_n \_S i_{3} N_{4}=4, n u c \_r \_S i_{3} N_{4}=4, t \_S i C \_i n t e r=5$, and $\left.t \_S i C \_i n t r a=5\right)$.

By using the value of $\alpha=1$, we end up solving a conventional single objective problem $\left(S_{R}=-\bar{S}_{r}\right)$, not considering $\operatorname{vrt}\left[S_{r}\right]$. To generate the surrogate of the mean deformation energy, quadratic response surface polynomials are created from samples obtained using the fractional factorial design method. As mentioned before, uncertainty is quantified on each sample by performing the response analysis 20 times.

Table 1 shows the results of the sequential approximate optimization under uncertainty algorithm. It details the current design $\left(x_{n}\right)$ and candidate design $\left(x_{n}{ }^{*}\right)$ per iteration, showing that the optimum microstructure is given by the design $x^{*}=[10,1,1,1,4,3,2]$. It can be seen that most of the design variables moved to their lower bounds. These results seem reasonable, since Fig. 1 shows how the strength of $\mathrm{Si}_{3} \mathrm{~N}_{4}$ is higher than the one of SiC. Table 1 also shows values for the response surface approximation $S_{R}$, and of the true objective function $S_{f}$ until convergence is reached. Values for the trust region ratio $(\rho)$, convergence parameters $\left(\varepsilon_{f 1}, \varepsilon_{f 2}, \varepsilon_{x}\right)$, number of samples to generate the RSA and to test the TR test, and number of evaluations per sample needed per iteration are shown as well, see Table 2 . Note that at iteration 4 the trust region test fails, then the current design is revaluated and in coming iterations the mean deformation energy

$\left(\bar{S}_{r}\right)$ is quantified in the samples need to construct the RSA. From Table 2, the total number of function evaluations (simulations) is 2445. This represents a lower number of function calls, compared to a global response surface that could be created from a full factorial design 
$\left(2^{7} \times 20=2560\right)$. Figure 6 shows the optimum microstructure that maximizes the deformation energy (area under force-displacement curve) at $1500{ }^{\circ} \mathrm{C}$.

Table 1. Results for $\alpha=1$, with initial design $x_{o}=[20,10,10,4,4,5,5]$, and deformation energy $S_{f}=0.039321 \mathrm{~J}$.

\begin{tabular}{|l|l|l|l|l|}
\hline Iteration & $x_{n}$ & $x_{n}{ }^{*}$ & $S_{R}(J)$ & $S_{f}(J)$ \\
\hline 1 & {$[20,10,10,4,4,5,5]$} & {$[16,6,8,5,5,4,4]$} & 0.039628 & 0.039414 \\
\hline 3 & {$[16,6,8,5,5,4,4]$} & {$[12,2,7,1,5,4,3]$} & 0.039552 & 0.039482 \\
\hline 4 & {$[12,2,7,1,5,4,3]$} & {$[10,1,1,1,4,3,2]$} & 0.039540 & 0.039530 \\
\hline 4 & {$[10,1,1,1,4,3,2]$} & {$[10,1,1,1,5,2,4]$} & 0.039535 & 0.039528 \\
\hline 5 & {$[10,1,1,1,4,3,2]$} & RSA $)$ & & \\
\hline 6 & {$[10,1,1,1,4,3,2]$} & {$[13,1,1,1,1,5,2]$} & 0.039549 & 0.039497 \\
\hline
\end{tabular}

Table 2. Trust region ratio value, convergence parameter values and number of function evaluations in the sequential approximate optimization under uncertainty algorithm (Case A).

\begin{tabular}{|l|l|l|l|l|l|l|}
\hline Iteration & $\rho$ & $\varepsilon_{f 1}$ & $\varepsilon_{f 2}$ & $\varepsilon_{x}$ & (UQ & No. samples Test TR \\
& & & & & Evaluations/sample) & Evaluations/sample) \\
\hline
\end{tabular}




\begin{tabular}{|l|l|l|l|l|l|l|}
\hline 1 & 0.30 & $2.06 \times 10^{-4}$ & $8.94 \times 10^{-5}$ & 0.181 & $66(1)$ & $1(20)$ \\
\hline 2 & 0.49 & $6.78 \times 10^{-5}$ & $6.54 \times 10^{-5}$ & 0.186 & $49(1)$ & $1(20)$ \\
\hline 3 & 0.83 & $9.59 \times 10^{-6}$ & $4.66 \times 10^{-5}$ & 0.167 & $49(1)$ & $1(20)$ \\
\hline 4 & -0.41 & & - & - & $41(1)$ & $1(20)$ \\
\hline 4 & \multicolumn{6}{|l|}{ (revaluate design and quantify $\bar{S}_{r}$ to generate the RSA) } \\
\hline 5 & -1.80 & & - & - & $41(20)$ & $1(20)$ \\
\hline 6 & 25.09 & $\mathbf{3 . 2 4 \times 1 0 ^ { - 6 }}$ & $\mathbf{3 . 3 8 \times 1 0 ^ { - 6 }}$ & $\mathbf{0 . 0}$ & $65(20)$ & $1(20)$ \\
\hline
\end{tabular}

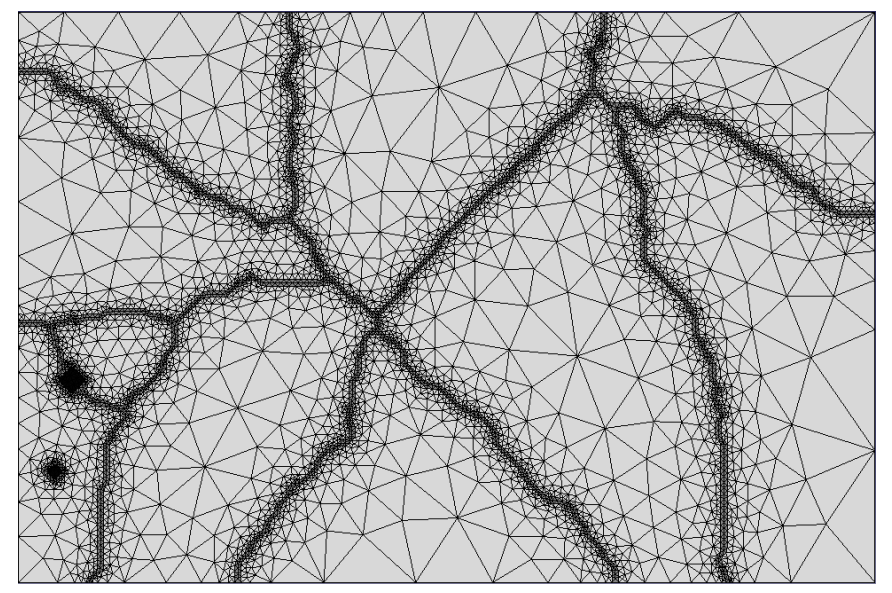

Figure 6. Optimum microstructure of the sequential approximate optimization under uncertainty algorithm at $1500{ }^{\circ} \mathrm{C}$, and $\alpha=1$.

Figure 7 shows a comparison of the response of bulk $\mathrm{Si}_{3} \mathrm{~N}_{4}$, bulk $\mathrm{SiC}$, and the optimum design shown in Table 1 and Fig. 6 . The optimum design shows a higher deformation energy and higher strength than the two bulk materials at $1500{ }^{\circ} \mathrm{C}$. This improvement in the properties comes mainly from the microstructure morphology, its grains size distribution and in lower importance 
by the $\mathrm{SiC}$ grains. Finally, we notice how the lower strength of the GB did not affect the whole composite strength. This is because of the optimal arrangement of the grains.

Figure 8 shows the behavior of the microstructural attributes throughout iterations in the optimization framework, for iterations where the TR test passes. Values of these attributes at the optimum design (Fig. 6) are: the volume fraction of the grain boundary is $3.9 \%$, of $\mathrm{Si}_{3} \mathrm{~N}_{4}$ is $95.9 \%$, of the intergranular $\mathrm{SiC}$ is $0.08 \%$ and of the intragranular $\mathrm{SiC}$ is $0.03 \%$. The grain size of $\mathrm{Si}_{3} \mathrm{~N}_{4}$ is $35860 \mu \mathrm{m}^{2}$, of the intergranular $\mathrm{SiC}$ is $298 \mu \mathrm{m}^{2}$ and of the intragranular $\mathrm{SiC}$ is $104 \mu \mathrm{m}^{2}$. The grain size distribution of $\mathrm{Si}_{3} \mathrm{~N}_{4}$ is $\beta=2.8$.

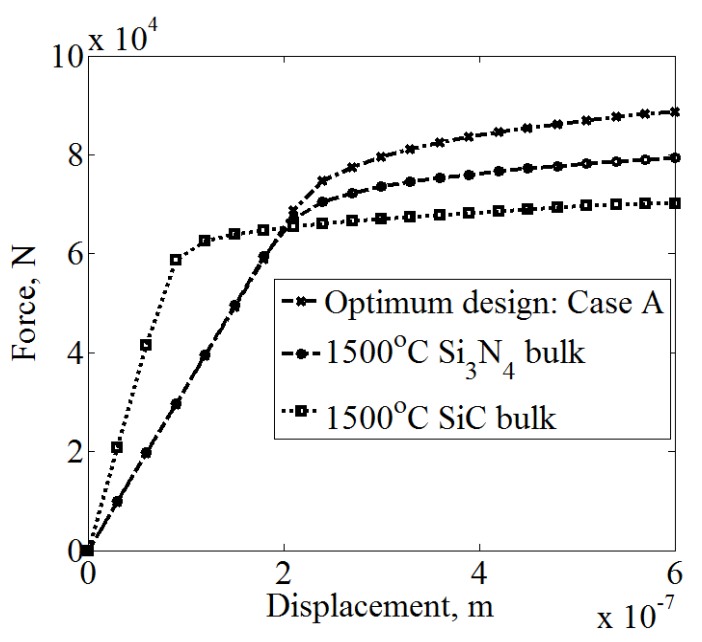

Figure 7. A comparison of force-displacement curve for the optimum microstructure with those for $\mathrm{Si}_{3} \mathrm{~N}_{4}$ and $\mathrm{SiC}$.

\section{B. Temperature $=1500{ }^{\circ} \mathrm{C}$ and $\alpha=0.5$}

In this case, two surrogate models are created, and same importance is given to both, the conventional objective (mean) and its variation (standard deviation). To generate the surrogate of the mean deformation energy and its standard deviation, quadratic response surface polynomials are created from samples obtained using the fractional factorial design method. As mentioned before, uncertainty is quantified on each sample by performing the response analysis 20 times. 
Table 3 shows the results of the sequential approximate optimization under uncertainty algorithm. To reduce computational time, results from Case A at iteration 3 are used as the starting design in this case, just before mean values of the deformation energy are used to construct the RSA. Table 3 shows that the robust optimum microstructure is given by the design $x^{*}=[10,1,1,3,4,3,2]$. We notice how the robust optimum design is very similar to the conventional design (Case A), where variations in the objective function are not considered. The authors realize the standard deviations are on the order of $1 \times 10^{-5}$, and these small values are the cause of such similarities in the results. Values for the trust region ratio $(\rho)$, convergence parameters $\left(\varepsilon_{f 1}, \varepsilon_{f 2}, \varepsilon_{x}\right)$, number of samples to generate the RSA and to test the TR test, and number of evaluations per sample needed per iteration are shown as well, see Table 4. As seen in Tables 3-4 convergence is reached in one iteration. This fast rate of convergence is due to having taken the starting design from Case A. Note that the fact of taking results from Case A to initialize this case B does not mean that the same path to the optimum design would have been taken by the optimizer, if the starting design from Case A had been considered, but it had been very similar because of the small values of the variations.

Table 3. Results for $\alpha=0.5$, with initial design $x_{o}=[10,1,1,1,4,3,2]$, and deformation energy $S_{f}=0.0395237 \mathrm{~J}$.

\begin{tabular}{|l|l|l|l|l|}
\hline Iteration & $x_{n}$ & $x_{n}{ }^{*}$ & $S_{R}(J)$ & $S_{f}(J)$ \\
\hline 1 & {$[10,1,1,1,4,3,2]$} & {$[\mathbf{1 0 , 1} \mathbf{1}, \mathbf{3}, \mathbf{4}, \mathbf{3}, \mathbf{2}]$} & 0.0395253 & $\mathbf{0 . 0 3 9 5 2 3 9}$ \\
\hline 2 & {$[10,1,1,3,4,3,2]$} & {$[10,1,1,3,4,3,2]$} & 0.0395251 & 0.0395238 \\
\hline
\end{tabular}


Table 4. Trust region ratio value, convergence parameter values and number of function evaluations in the sequential approximate optimization under uncertainty algorithm (Case B).

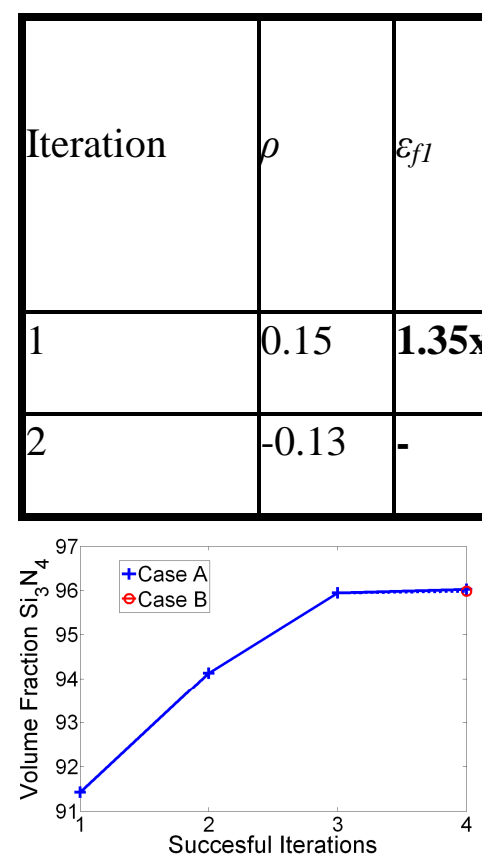

a)

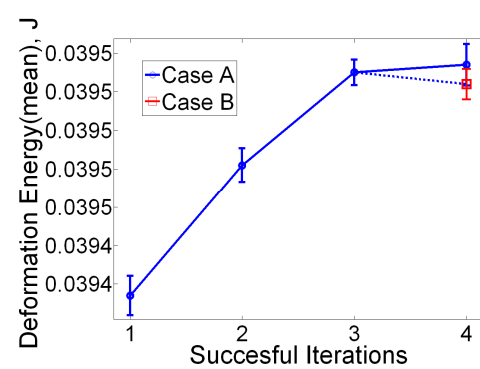

d)

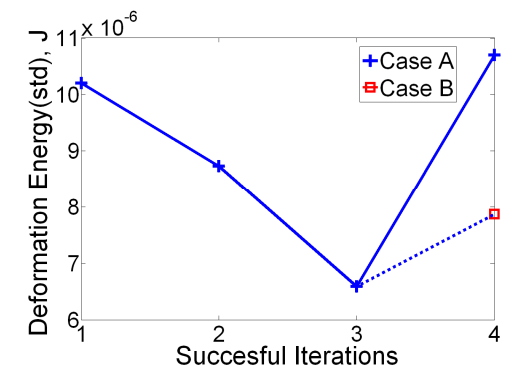

e)

Figure 8. Behavior of microstructural attributes, and behavior of the mean and standard deviation (std) of the objective function, throughout successful iterations (designs accepted at TR test, $\rho>0$ ) in cases A-B. 
In Fig. 8 results for Case B are shown at iteration 4, to compare with results of case A. Figure 8 shows, a) how the volume fraction of $\mathrm{Si}_{3} \mathrm{~N}_{4}$ increases through iterations in the algorithm, b) on the other hand the volume fraction of $\mathrm{SiC}$ (intergranular and intragranular) decreases as the algorithm converges. Also, it is noticed, c) that the size ratios of the SiC (intergranular and intragranular) grains over the size of the $\mathrm{Si}_{3} \mathrm{~N}_{4}$ grains decrease for case $\mathrm{A}$, but for case $\mathrm{B}$ they increase by a small amount with respect to case A. In d) the mean deformation energy is shown along with its \pm standard deviation. An increase in the mean deformation energy is shown, which decreases by a small amount for the robust problem (case B). Also, e) a decrease in the standard deviation of the deformation energy is observed, and it is shown how it decreases for the robust problem (Case B) with respect to Case A at the optimum.

\section{Temperature $=1600{ }^{\circ} \mathrm{C}$ and $\alpha=1$}

In this case, the initial design is $x_{o}=[25,15,15,4,4,5,5]\left(n \_S i_{3} N_{4}=25, n \_S i C \_i n t e r=15\right.$, $n \_S i C \_i n t r a=15, i n \_n \_S i_{3} N_{4}=4, n u c \_r \_S i_{3} N_{4}=4, t \_S i C \_i n t e r=5$, and $\left.t \_S i C \_i n t r a=5\right)$.

By using the value of $\alpha=1$, we end up solving a conventional single objective problem $\left(S_{R}=-\bar{S}_{r}\right)$, not considering $\operatorname{vrt}\left[S_{r}\right]$. To generate the surrogate of the mean deformation energy, quadratic response surface polynomials are created from samples obtained using the fractional factorial design method. As mentioned before, uncertainty is quantified on each sample by performing the response analysis 20 times.

Table 5 shows the results of the sequential approximate optimization under uncertainty algorithm. It details the current design $\left(x_{n}\right)$ and candidate design $\left(x_{n}{ }^{*}\right)$ per iteration, showing that the optimum microstructure is given by the design $x^{*}=[40,25,36,21,10,8,8]$. It can be seen that most of the design variables moved to their upper bounds. These results seem reasonable, since Fig. 1 shows how the strength of $\mathrm{SiC}$ is higher than the one of $\mathrm{Si}_{3} \mathrm{~N}_{4}$. Table 3 also shows 
values for the response surface approximation $S_{R}$, and of the true objective function $S_{f}$ until convergence is reached. Values for the trust region ratio $(\rho)$, convergence parameters $\left(\varepsilon_{f 1}, \varepsilon_{f 2}, \varepsilon_{x}\right)$, number of samples to generate the RSA and to test the TR test, and number of evaluations per sample needed per iteration are shown as well, see Table 6 . Note that at iteration 8 the trust region test fails, then the current design is revaluated and in coming iterations the mean deformation energy $\left(\bar{S}_{r}\right)$ is quantified in the samples need to construct the RSA. From Table 6 , the total number of function evaluations (simulations) is 2802. This represents a higher number of function calls, compared to a global response surface that could be created from a full factorial design $\left(2^{7} \times 20=2560\right)$. The increase in the number of evaluations is due to the difficulties to create an accurate surrogate model in the upper limit of the design variables. Figure 9 shows the optimum microstructure that maximizes the deformation energy (area under force-displacement curve) at $1600{ }^{\circ} \mathrm{C}$. 
Table 5. Results for $\alpha=1$, with initial design $x_{0}=[25,15,15,4,4,5,5]$, and deformation energy $S_{f}=0.033152 \mathrm{~J}$.

\begin{tabular}{|c|c|c|c|c|}
\hline Iteration & $\overline{x_{n}}$ & $x_{n}^{*}$ & $S_{R}(J)$ & $\overline{S_{f}(J)}$ \\
\hline 1 & {$[25,15,15,4,4,5,5]$} & {$[21,19,21,5,5,6,6]$} & 0.035251 & 0.033768 \\
\hline 2 & {$[21,19,21,5,5,6,6]$} & {$[23,23,23,4,6,7,7]$} & 0.034218 & 0.034141 \\
\hline 3 & {$[23,23,23,4,6,7,7]$} & {$[32,21,28,1,7,8,8]$} & 0.034765 & 0.034341 \\
\hline 4 & {$[32,21,28,1,7,8,8]$} & {$[40,19,35,10,10,8,8]$} & 0.034661 & 0.034408 \\
\hline 5 & {$\left[\begin{array}{l}{[40,19,35,10,10,8,} \\
8]\end{array}\right.$} & {$[40,19,35,13,10,8,8]$} & 0.034639 & 0.034480 \\
\hline 6 & {$[40,19,35,13,10,8$,} & {$[40,19,35,19,10,8,8]$} & 0.034663 & 0.034485 \\
\hline 7 & {$[40,19,35,19,10,8$,} & {$[40,24,35,21,10,8,8]$} & 0.034706 & 0.034534 \\
\hline 8 & {$[40,24,35,21,10,8$,} & {$[40,25,36,21,9,7,7]$} & 0.072728 & 0.034274 \\
\hline 8 & {$[40,24,35,21,10,8$,} & \multicolumn{3}{|c|}{ (revaluate design and quantify $\bar{S}_{r}$ to generate the RSA) } \\
\hline 9 & {$[40,24,35,21,10,8$,} & {$[36,23,29,21,10,8,8]$} & 0.034707 & 0.034502 \\
\hline 10 & {$[40,24,35,21,10,8$} & {$[40,25,36,21,10,8,8]$} & 0.034574 & 0.034569 \\
\hline
\end{tabular}


Table 6. Trust region ratio value, convergence parameter values and number of function evaluations in the sequential approximate optimization under uncertainty algorithm (Case C).

\begin{tabular}{|c|c|c|c|c|c|c|}
\hline Iteration & $p$ & $\varepsilon_{f 1}$ & $\varepsilon_{f 2}$ & $\varepsilon_{x}$ & $\begin{array}{l}\text { No. samples RSA } \\
\text { (UQ } \\
\text { Evaluations/sample) }\end{array}$ & $\begin{array}{l}\text { No. samples Test TR } \\
\text { (UQ } \\
\text { Evaluations/sample) }\end{array}$ \\
\hline 1 & 0.29 & $1.43 \times 10^{-3}$ & $5.97 \times 10^{-4}$ & 0.18 & $66(1)$ & $1(20)$ \\
\hline 2 & 0.83 & $7.45 \times 10^{-5}$ & $3.61 \times 10^{-4}$ & 0.19 & $33(1)$ & $1(20)$ \\
\hline 3 & 0.32 & $4.10 \times 10^{-4}$ & $1.93 \times 10^{-4}$ & 0.33 & $25(1)$ & $1(20)$ \\
\hline 4 & 0.21 & $2.45 \times 10^{-4}$ & $6.49 \times 10^{-5}$ & 0.33 & $49(1)$ & $1(20)$ \\
\hline 5 & 0.31 & $1.54 \times 10^{-4}$ & $6.91 \times 10^{-5}$ & 0.08 & $65(1)$ & $1(20)$ \\
\hline 6 & 0.03 & $1.72 \times 10^{-4}$ & $4.88 \times 10^{-6}$ & 0.15 & $50(1)$ & $1(20)$ \\
\hline 7 & 0.22 & $1.65 \times 10^{-4}$ & $4.77 \times 10^{-5}$ & 0.21 & $49(1)$ & $1(20)$ \\
\hline 8 & -0.01 & {[} & - & $F$ & $65(1)$ & $1(20)$ \\
\hline 8 & (reval & uate design & and quant & tify ? & to generate the RSA & \\
\hline 9 & -0.19 & 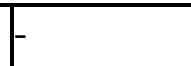 & F & - & 49 (20) & $1(20)$ \\
\hline$\sqrt{10}$ & 0.88 & $4.77 \times 10^{-6}$ & $3.38 \times 10^{-5}$ & 0.04 & $61(20)$ & $1(20)$ \\
\hline
\end{tabular}




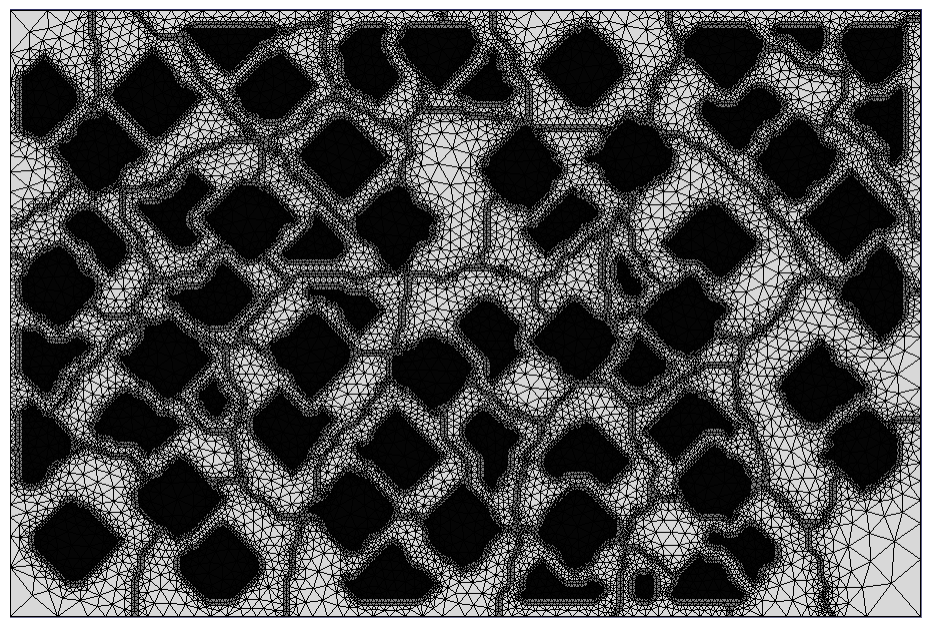

Figure 9. Optimum microstructure of the sequential approximate optimization under uncertainty algorithm at $1600{ }^{\circ} \mathrm{C}$, and $\alpha=1$.

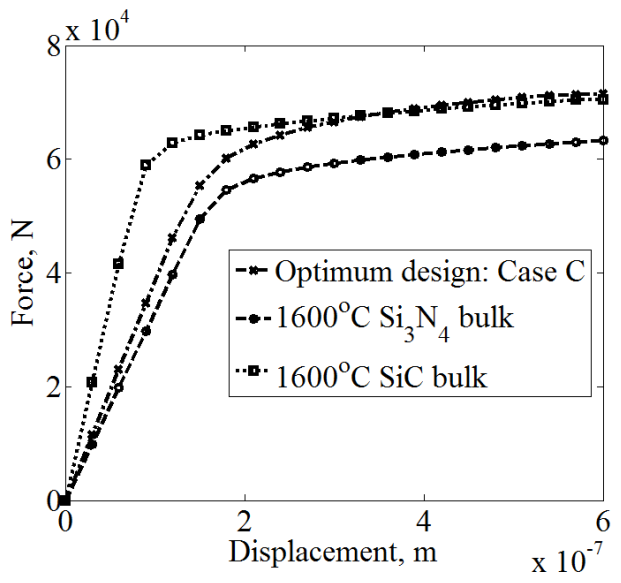

Figure 10. A comparison of force-displacement curve for the optimum microstructure with those for $\mathrm{Si}_{3} \mathrm{~N}_{4}$ and $\mathrm{SiC}$.

Figure 10 shows a comparison of the response of bulk $\mathrm{Si}_{3} \mathrm{~N}_{4}$, bulk $\mathrm{SiC}$, and the optimum design shown in Table 5 and Fig. 9. The optimum design shows a higher ultimate strength than 
the two bulk materials at $1600{ }^{\circ} \mathrm{C}$, and a promising higher deformation energy at higher strains. This improvement in the properties comes from the microstructure morphology, the grains size distribution of the $\mathrm{Si}_{3} \mathrm{~N}_{4}$ grains and by the intergranular and intragranular $\mathrm{SiC}$ grains.

Figure 11 shows the behavior of the microstructural attributes throughout iterations in the optimization framework, for iterations where the TR test passes. Values of these attributes at the optimum design (Fig. 9) are: the volume fraction of the grain boundary is $19.5 \%$, of $\mathrm{Si}_{3} \mathrm{~N}_{4}$ is $52.4 \%$, of the intergranular $\mathrm{SiC}$ is $12.4 \%$ and of the intragranular $\mathrm{SiC}$ is $16.3 \%$. The grain size of $\mathrm{Si}_{3} \mathrm{~N}_{4}$ is $4838 \mu \mathrm{m}^{2}$, of the intergranular $\mathrm{SiC}$ is $2246 \mu \mathrm{m}^{2}$ and of the intragranular $\mathrm{SiC}$ is $1546 \mu \mathrm{m}^{2}$. The grain size distribution of $\mathrm{Si}_{3} \mathrm{~N}_{4}$ is $\beta=1.6$.

\section{Temperature $=1600{ }^{\circ} \mathrm{C}$ and $\alpha=0.5$}

In this case, two surrogate models are created, and same importance is given to both, the conventional objective (mean) and its variation (standard deviation). To generate the surrogate of the mean deformation energy and its standard deviation, quadratic response surface polynomials are created from samples obtained using the fractional factorial design method. As mentioned before, uncertainty is quantified on each sample by performing the response analysis 20 times.

Table 7 shows the results of the sequential approximate optimization under uncertainty algorithm. To reduce computational time, results from Case $\mathrm{C}$ at iteration 7 are used as the starting design in this case, just before mean values of the deformation energy are used to construct the RSA. The table shows that the robust optimum microstructure is given by the design $x^{*}=[40,24,36,21,9,8,8]$. Again, we notice how the robust optimum design is very similar to the conventional design (Case C), where variations in the objective function are not

considered. The authors realize the standard deviations are on the order of $5 \times 10^{-5}$, and these small values are the cause of such similarities in the results. Values for the trust region ratio $(\rho)$, 
convergence parameters $\left(\varepsilon_{f 1}, \varepsilon_{f 2}, \varepsilon_{x}\right)$, number of samples to generate the RSA and to test the TR test, and number of evaluations per sample needed per iteration are shown as well, see Table 8 . As seen in Tables 7-8 convergence is reached in three iterations. This fast rate of convergence is due to having taken the starting design from Case C. Note that the fact of taking results from Case $\mathrm{C}$ to initialize this case $\mathrm{D}$ does not mean that the same path to the optimum design would have been taken by the optimizer, if the starting design from Case $\mathrm{C}$ had been considered, but it had been very similar because of the small values of the variations.

Table 7. Results for $\alpha=0.5$, with initial design $x_{o}=[40,24,35,21,10,8,8]$, and deformation energy $S_{f}=0.034531 \mathrm{~J}$.

\begin{tabular}{|c|c|c|c|c|}
\hline Iteration & $x_{n}$ & $x_{n}^{*}$ & $S_{R}(J)$ & $S_{f}(J)$ \\
\hline 1 & $\begin{array}{l}{[40,24,35,21,10,8,} \\
8]\end{array}$ & {$[36,21,29,18,9,8,8]$} & 0.035187 & 0.034386 \\
\hline 2 & $\begin{array}{l}{[40,24,35,21,10,8,} \\
8]\end{array}$ & {$[40,24,36,21,9,8,8]$} & 0.034566 & 0.034590 \\
\hline 3 & $\begin{array}{l}{[40,24,36,21,9,8,} \\
8]\end{array}$ & {$[40,24,36,21,9,8,8]$} & 0.034595 & 0.034597 \\
\hline
\end{tabular}

Table 8. Trust region ratio value, convergence parameter values and number of function evaluations in the sequential approximate optimization under uncertainty algorithm (Case D).

\begin{tabular}{|c|c|c|c|c|c|c|}
\hline Iteration & $p$ & $\varepsilon_{f l}$ & $\varepsilon_{f 2}$ & $\varepsilon_{x}$ & $\begin{array}{l}\text { No. samples RSA } \\
\text { (UQ }\end{array}$ & $\begin{array}{l}\text { No. samples Test TR } \\
\text { (UQ }\end{array}$ \\
\hline
\end{tabular}




\begin{tabular}{|c|c|c|c|c|c|c|}
\hline & & & & & Evaluations/sample) & Evaluations/sample) \\
\hline 1 & -0.23 & - & - & - & $49(20)$ & 1 (20) \\
\hline 2 & 10.22 & $2.38 \times 10^{-5}$ & $2.63 \times 10^{-5}$ & 0.11 & $65(20)$ & $1(20)$ \\
\hline 3 & 1.52 & $2.31 \times 10^{-6}$ & $6.77 \times 10^{-6}$ & 0.0 & $62(20)$ & $1(20)$ \\
\hline
\end{tabular}
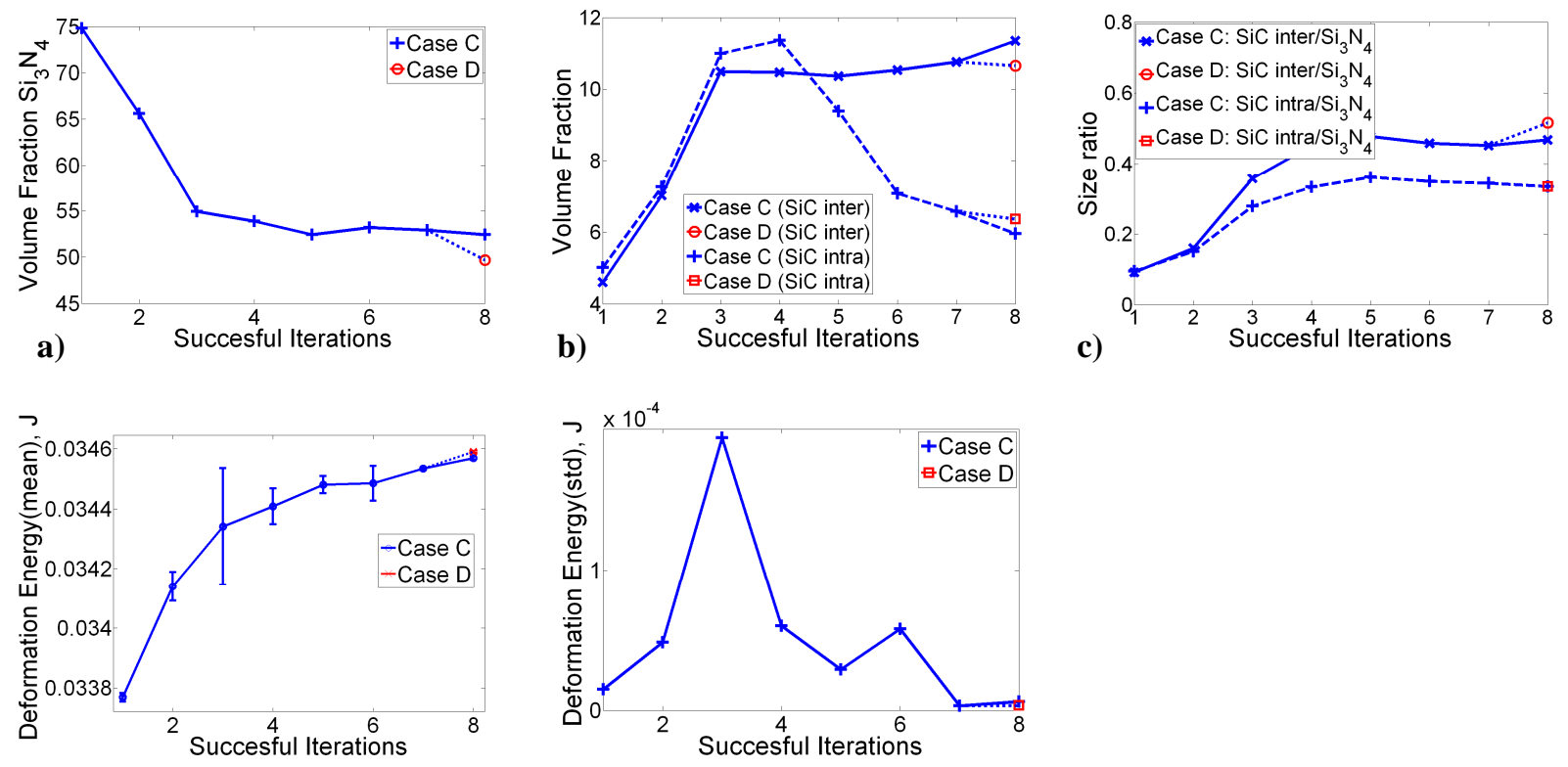

d)

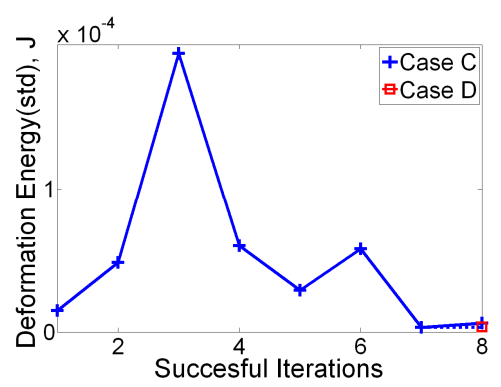

e)

Figure 11. Behavior of microstructural attributes, and behavior of the mean and standard deviation (std) of the objective function, throughout successful iterations (designs accepted at TR test, $\rho>0)$ in cases C-D.

In Fig. 11 results at the optimum for Case $\mathrm{D}$ are shown at iteration 8 , to compare with results of case C. Figure 11, a) shows how the volume fraction of $\mathrm{Si}_{3} \mathrm{~N}_{4}$ decreases through iterations in the algorithm, b) on the other hand the volume fraction of $\mathrm{SiC}$ (intergranular and intragranular) increases as the algorithm converges for case $\mathrm{C}$, but for the robust problem (case D) the 
intergranular $\mathrm{SiC}$ increases and the intragranular $\mathrm{SiC}$ decreases. For the $\mathrm{SiC}$ size ratios c), a similar behavior is observed to the one of the volume fraction. In d) an increase in the mean deformation energy is shown along with its \pm standard deviation. Finally, e) a decrease in the standard deviation of the deformation energy is observed, and it is shown how it decreases for the robust problem with respect to Case $\mathrm{C}$ at the optimum.

\section{E. Equal Weighted Sum at Both Temperatures $\left(1500{ }^{\circ} \mathrm{C}\right.$ and $\left.1600{ }^{\circ} \mathrm{C}\right)$, and $\alpha=1$}

In the previous four cases (A-D), optimum microstructures have been obtained to be used at $1500{ }^{\circ} \mathrm{C}$, and at $1600^{\circ} \mathrm{C}$. But what about a material that is desired to operate at a range of different temperatures. That possibility is considered by giving same importance to both temperatures $\left(1500{ }^{\circ} \mathrm{C}\right.$ and $\left.1600^{\circ} \mathrm{C}\right)$. The approach taken is to equally weight the composite response at both temperatures, using a multiobjective optimization formulation.

By using $\alpha=1$, we end up solving a multiobjective problem on $T$ (not considering $\operatorname{vrt}\left[S_{r}\right]$ ), with the form:

$$
S_{r}=-0.5 \cdot \bar{S}_{r}\left(T=1500^{\circ} \mathrm{C}\right)-0.5 \cdot \bar{S}_{r}\left(T=1600^{\circ} \mathrm{C}\right)
$$

To generate the two surrogates of the mean deformation energy, quadratic response surface polynomials are created from samples obtained using fractional factorial design method. As mentioned before, uncertainty is quantified on each sample by performing the response analysis 20 times.

In this case, the initial design is $x_{o}=[25,10,15,10,5,5,5]\left(n \_S i_{3} N_{4}=25, n \_S i C \_i n t e r=10\right.$, $n \_S i C \_i n t r a=15$, in_n_Si $i_{3} N_{4}=10, n u c \_r \_S i_{3} N_{4}=5, t \_S i C \_i n t e r=5$, and $\left.t \_S i C \_i n t r a=5\right)$. Table 9 shows the results of the sequential approximate optimization under uncertainty algorithm. It details the current design $\left(x_{n}\right)$ and candidate design $\left(x_{n}{ }^{*}\right)$ per iteration, showing that the optimum microstructure is given by the design $x^{*}=[10,19,9,7,3,8,8]$. This design results in a more 
intermediate microstructure, compared to the results from cases A-D where designs did not show compromised microstructures.

Table 9 also shows values for the response surface approximation $S_{R}$, of the true objective function $S_{f}$ until convergence is reached. In this case the values of the true and approximate functions are normalized to have a value between 0 and 1. Values for the trust region ratio $(\rho)$, convergence parameters $\left(\varepsilon_{f 1}, \varepsilon_{f 2}, \varepsilon_{x}\right)$, number of samples to generate the RSA and to test the TR test, and number of evaluations per sample needed per iteration are shown as well, see Table 10. Note that at iteration 8 the trust region test fails, then the current design is revaluated and in coming iterations the mean deformation energy $\left(\bar{S}_{r}\right)$ is quantified in the samples need to construct the RSA. From Table 10, the total number of function evaluations (simulations) is 1558(for one objective). This represents a lower number of function calls, compared to a global response surface that could be created from full factorial design $\left(2^{7} \times 20=2560\right.$ for one objective $)$. Figure 12 shows the optimum microstructure that maximizes the deformation energy (area under force-displacement curve) at $1500{ }^{\circ} \mathrm{C}$ and $1600{ }^{\circ} \mathrm{C}$ simultaneously.

Table 9. Results for $\alpha=1$, with initial design $x_{o}=[25,10,15,10,5,5,5]$, and deformation energy $S_{f}=0.427 \mathrm{~J}$.

\begin{tabular}{|l|l|l|l|l|}
\hline Iteration & $x_{n}$ & $x_{n}{ }^{*}$ & $S_{R}(J)$ & $S_{f}(J)$ \\
\hline 1 & {$[25,10,15,10,5,5,5]$} & {$[25,14,15,4,4,6,6]$} & 0.653 & 0.467 \\
\hline 3 & {$[25,14,15,4,4,6,6]$} & {$[25,15,16,3,5,7,7]$} & 0.538 & 0.535 \\
\hline 4 & {$[25,15,16,3,5,7,7]$} & {$[23,17,19,1,5,8,8]$} & 0.660 & 0.650 \\
\hline 5 & {$[23,17,19,1,5,8,8]$} & {$[19,17,19,1,5,8,8]$} & 0.712 & 0.668 \\
\hline
\end{tabular}




\begin{tabular}{|l|l|l|l|l|}
\hline 6 & {$[15,17,15,7,4,8,8]$} & {$[11,17,11,7,4,8,8]$} & 0.756 & 0.700 \\
\hline 7 & {$[11,17,11,7,4,8,8]$} & {$[10,18,10,6,4,8,8]$} & 0.718 & 0.702 \\
\hline 8 & {$[10,18,10,6,4,8,8]$} & {$[10,18,10,6,4,8,7]$} & 0.731 & 0.681 \\
\hline 8 & {$[10,18,10,6,4,8,8]$} & (revaluate design and quantify $\bar{S}_{r}$ to generate the RSA) \\
\hline 9 & {$[10,18,10,6,4,8,8]$} & {$[\mathbf{1 0 , 1 9 , 9 , 7 , 3 , 8 , 8}]$} & 0.707 & $\mathbf{0 . 7 0 4}$ \\
\hline
\end{tabular}

Table 10. Trust region ratio value, convergence parameter values and number of function evaluations in the sequential approximate optimization under uncertainty algorithm (Case E).

\begin{tabular}{|c|c|c|c|c|c|c|}
\hline Iteration & $p$ & $\varepsilon_{f 1}$ & $\varepsilon_{f 2}$ & $\varepsilon_{x}$ & $\begin{array}{l}\text { No. samples RSA } \\
\text { (UQ } \\
\text { Evaluations/sample) }\end{array}$ & $\begin{array}{l}\text { No. sample Test TR } \\
\text { (UQ } \\
\text { Evaluations/sample) }\end{array}$ \\
\hline $\mid 1$ & 0.18 & $1.27 \times 10^{-1}$ & $2.82 \times 10^{-2}$ & 0.22 & $66 \times 2(1)$ & $2(20)$ \\
\hline 2 & 0.96 & $2.02 \times 10^{-3}$ & $4.64 \times 10^{-2}$ & 0.18 & $65 \times 2(1)$ & $2(20)$ \\
\hline 3 & 0.92 & $6.21 \times 10^{-3}$ & $7.49 \times 10^{-2}$ & 0.18 & $65 \times 2(1)$ & $2(20)$ \\
\hline 4 & 0.28 & $2.67 \times 10^{-2}$ & $1.07 \times 10^{-2}$ & 0.13 & $49 \times 2(1)$ & $2(20)$ \\
\hline 5 & 0.33 & $1.96 \times 10^{-2}$ & $9.73 \times 10^{-3}$ & 0.15 & $33 \times 2(1)$ & $2(20)$ \\
\hline 6 & 0.23 & $3.28 \times 10^{-2}$ & $9.66 \times 10^{-3}$ & 0.13 & $25 \times 2(1)$ & $2(20)$ \\
\hline 7 & 0.11 & $9.12 \times 10^{-3}$ & $1.10 \times 10^{-3}$ & 0.04 & $48 \times 2(1)$ & $2(20)$ \\
\hline 8 & -0.71 & - & - & F & $47 \times 2(1)$ & $2(20)$ \\
\hline 8 & (reval & uate design & and quantil & $\overline{S_{r}}$ to & senerate the RSA) & \\
\hline 9 & 0.28 & $2.4 \times 10^{-3}$ & $9.21 \times 10^{-4}$ & 0.11 & $49 \times 2(20)$ & $2(20)$ \\
\hline
\end{tabular}




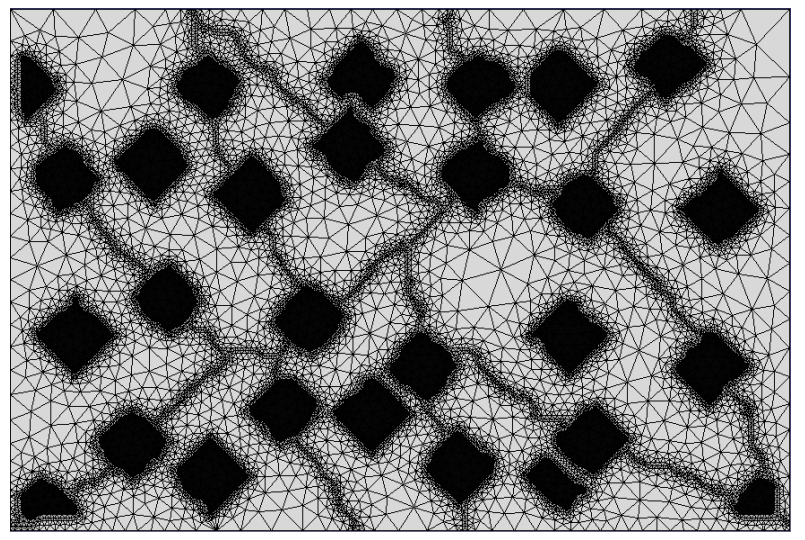

Figure 12. Multiobjective based optimum microstructure of the sequential approximate optimization under uncertainty algorithm with $\alpha=1$.

Figure 13 shows the behavior of the microstructural attributes throughout iterations in the optimization framework, for iterations where the TR test passes. Values of these attributes at the optimum design (Fig. 12) are: the volume fraction of the grain boundary is $9.2 \%$, of $\mathrm{Si}_{3} \mathrm{~N}_{4}$ is $73.5 \%$, of the intergranular $\mathrm{SiC}$ is $11.3 \%$ and of the intragranular $\mathrm{SiC}$ is $6.0 \%$. The grain size of $\mathrm{Si}_{3} \mathrm{~N}_{4}$ is $27462 \mu \mathrm{m}^{2}$, of the intergranular $\mathrm{SiC}$ is $2232 \mu \mathrm{m}^{2}$ and of the intragranular $\mathrm{SiC}$ is $2472 \mu \mathrm{m}^{2}$. The grain size distribution of $\mathrm{Si}_{3} \mathrm{~N}_{4}$ is $\beta=3.6$.

\section{F. Equal Weighted Sum at Both Temperatures $\left(1500{ }^{\circ} \mathrm{C}\right.$ and $\left.1600{ }^{\circ} \mathrm{C}\right)$, and $\alpha=0.5$}

By using $\alpha=0.5$, we end up solving a multiobjective problem on $T$, with the form:

$$
S_{r}=\left[-0.25 \alpha \bar{S}_{r}+0.25 \operatorname{vrt}\left[S_{r}\right]\right]\left(T=1500^{\circ} \mathrm{C}\right)+\left[-0.25 \bar{S}_{r}+0.25 \operatorname{vrt}\left[S_{r}\right]\right]\left(T=1600^{\circ} \mathrm{C}\right)
$$

In this case, four surrogate models are created, and same importance is given to both, the conventional objectives (mean) and their variation (standard deviation). To generate the surrogates of the mean deformation energy and their standard deviation, quadratic response 
surface polynomials are created from samples obtained using the fractional factorial design method. As mentioned before, uncertainty is quantified on each sample by performing the response analysis 20 times.

Table 11 shows the results of the sequential approximate optimization under uncertainty algorithm. To reduce computational time, results from Case $\mathrm{E}$ at iteration 7 are used as the starting design in this case, just before mean values of the deformation energy are used to construct the RSA. The table shows that the robust optimum microstructure is given by the design $x^{*}=[10,18,10,7,3,8,8]$. Also, the results show how the robust optimum design is very similar to the conventional design (case E), where variations in the objective function are not considered. The authors realize the standard deviations are on the order of $3 \times 10^{-5}$, and these small values are the cause of such similarities in the results. Values for the trust region ratio $(\rho)$, convergence parameters $\left(\varepsilon_{f 1}, \varepsilon_{f 2}, \varepsilon_{x}\right)$, number of samples to generate the RSA and to test the TR test, and number of evaluations per sample needed per iteration are shown as well, see Table 12. As seen in Tables 11-12 convergence is reached in two iterations. This fast rate of convergence is due to having taken the starting design from Case E. Note that the fact of taking results from Case $\mathrm{E}$ to initialize this case $\mathrm{F}$ does not mean that the same path to the optimum design would have been taken by the optimizer, if the starting design from Case $\mathrm{E}$ had been considered, but it had been very similar because of the small values of the variations. 
Table 11. Results for $\alpha=0.5$, with initial design $x_{o}=[10,18,10,6,4,8,8]$, and deformation energy $S_{f}=0.68422 \mathrm{~J}$.

\begin{tabular}{|l|l|l|l|l|}
\hline Iteration & $x_{n}$ & $x_{n}^{*}$ & $S_{R}$ & $S_{f}$ \\
\hline 1 & {$[10,18,10,6,4,8,8]$} & {$[10,18,10,7,3,8,8]$} & 0.79121 & 0.69863 \\
\hline 2 & {$[10,18,10,7,3,8,8]$} & {$[\mathbf{1 0 , 1 8 , 1 0 , 7 , 3 , 8 , 8 ]}$} & 0.72587 & $\mathbf{0 . 6 9 8 6 5}$ \\
\hline
\end{tabular}

Table 12. Trust region ratio value, convergence parameter values and number of function evaluations in the sequential approximate optimization under uncertainty algorithm (Case F).

\begin{tabular}{|l|l|l|l|l|l|l|}
\hline \multirow{2}{*}{ Iteration } & $\rho$ & & & & No. samples RSA & No. samples Test TR \\
& & & $\varepsilon_{f 1}$ & $\varepsilon_{x}$ & (UQ & (UQ \\
\hline 1 & 0.13 & $5.45 \times 10^{-2}$ & $8.55 \times 10^{-3}$ & 0.13 & $49 \times 2(20)$ & $2(20)$ \\
\hline 2 & 0.01 & $1.60 \times 10^{-2}$ & $\mathbf{1 . 2 4 \times 1 0 ^ { - 5 }}$ & $\mathbf{0 . 0}$ & $49 \times 2(20)$ & $2(20)$ \\
\hline
\end{tabular}



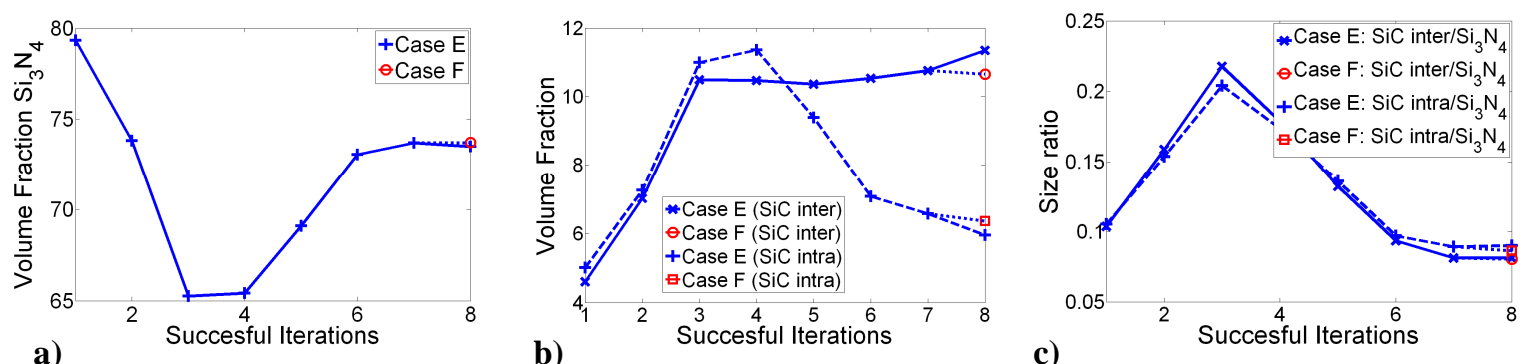

a)

b)

c)

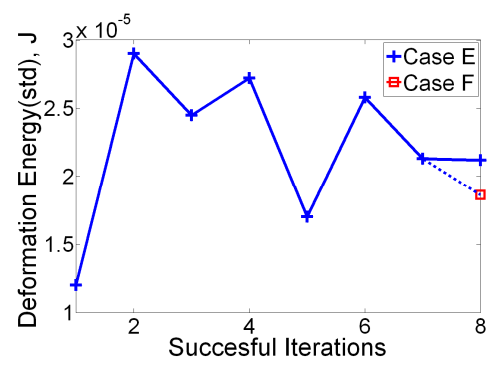

e)

\section{Figure 13. Behavior of microstructural attributes, and behavior of the mean and standard deviation (std) of the objective function, throughout successful iterations (designs accepted at TR test $\rho>0$ ) in cases E-F.}

In Fig. 13 results at the optimum for Case $\mathrm{F}$ are shown at iteration 8, to compare with results of case E. Figure 13 shows, a) how the volume fraction of $\mathrm{Si}_{3} \mathrm{~N}_{4}$ first decreases and then increases as the algorithm converges. In b), the intergranular $\mathrm{SiC}$ volume fraction increases for case $\mathrm{E}$, and then decreases by a small amount in $\mathrm{F}$. On the other hand, the intragranular $\mathrm{SiC}$ volume fraction decreases for case $\mathrm{E}$, and increases by a small amount in F. Also it is noticed, c) that the size ratios of the $\mathrm{SiC}$ (intergranular and intragranular) grains over the size of the $\mathrm{Si}_{3} \mathrm{~N}_{4}$ grains decrease as the algorithm converges. In d) the mean deformation energy is shown along with its \pm standard deviation. An increase in the mean deformation energy is shown, which decreases by a small amount for the robust problem (case F). Finally in e) a decrease in the standard deviation of the deformation energy is observed, and it is shown how it decreases for the robust problem with respect to Case $\mathrm{C}$ at the optimum. 
A bigger difference between the results from the conventional and the robust design approach could be seen if bigger values of uncertainty resulted from the microstructural and FE simulations. Bigger values of uncertainty could result by considering other types of uncertainties, such as uncertainties introduced from variations on the input microstructure parameters (design variables), or by using material models with higher physics that would result in microstructures with more design parameters. Also note that the results from cases $\mathrm{E}$ and $\mathrm{F}$ can be considered as pareto points in the optimization problem, and more points could be obtained values different from 0.5 were considered.

In this investigation, improvement in the deformation energy and strength of the nonocomposites arises from optimum microstructure morphologies, optimum grain size distribution of the $\mathrm{Si}_{3} \mathrm{~N}_{4}$ grains, and from the optimum location and size of the intergranular and intragranular $\mathrm{SiC}$ grains. The constitutive model of Eq. (14) has been used to model the deformation mechanism and bulk properties of silicon nitride $\left(\mathrm{Si}_{3} \mathrm{~N}_{4}\right)$, silicon carbide $(\mathrm{SiC})$ and an amorphous silicate based grain boundary (GB). In the model, the temperature effect on the flow stresses is accounted through the viscosity of silicon nitride and silicon carbide at elevated temperatures. At $1500{ }^{\circ} \mathrm{C}$ the preferred material is $\mathrm{Si}_{3} \mathrm{~N}_{4}$, on the other hand the preference is on $\mathrm{SiC}$ at $1600{ }^{\circ} \mathrm{C}$, in accordance with experimental predictions available in literature. Future work will focus on incorporating more strengthening and toughening mechanisms in the nanocomposites through higher physics models, such as cohesive fracture models, crystal plasticity models, atomistic models etc. 


\section{Conclusion}

This work introduced a simulation-based robust material design optimization methodology, to predict the most suitable microstructural phase morphologies for desired high temperature deformation energy of $\mathrm{SiC}-\mathrm{Si}_{3} \mathrm{~N}_{4}$ nanocomposites. Seven design variables were considered in the optimization formulation to search for improved deformation energy and strength of the nonocomposites. Improvement in the material properties arose from the optimum microstructure morphologies, grains size distribution of the $\mathrm{Si}_{3} \mathrm{~N}_{4}$ grains and by the intergranular and intragranular $\mathrm{SiC}$ grains. An approach was presented to reduce the computational time associated with UQ during the initial iterations of the sequential approximate optimization under uncertainty algorithm. The use of the sequential approximate optimization under uncertainty algorithm and the use of robust design optimization techniques in multiscale material design will allow developing $\mathrm{SiC}-\mathrm{Si}_{3} \mathrm{~N}_{4}$ nanocomposites whose performance variation is insensitive to processing variations, while reducing design cycle times. The material design tool was shown to be an effective methodology for generating feasible and optimal hybrid material morphologies for high temperature deformation energy. 


\section{V-Nanoscale and Mesoscale Creep Experiments ${ }^{4}$}

\section{§1. INTRODUCTION}

Creep is non-recoverable high temperature plastic deformation occurring at low load regimes, constant stress, and small strain rates. Characterization of creep deformation is very important in the case of materials intended for use at high temperatures. Another important creep deformation is the one occurring at nanoscale contacts at moderate temperatures. Characterization of such creep deformation is important for applications related to operation of miniature devices, thermal stability of interfaces etc. Indentation creep experiments with typical holding times of 500 seconds are used to characterize such creep deformation, [124-126]. Indentation creep is defined as the constant rate indenter displacement $(\dot{h})$ after a finite time of load hold $(\sim 500 \mathrm{sec})$ once the indenter displacement is free of thermal drift (usually for depths $\geq$ $200 \mathrm{~nm}$ ). Fundamentally, during nanoscale or microscale indentation tests contact stresses are high. Correspondingly, nominal pressure during nano- and microindentation can easily reach a few percent of the Young's modulus of materials resulting in an ideal strength situation. Due to such high stress contacts indentation creep can occur at low homologous temperatures (Current Temperature (T)/Melting Temperature $\left(\mathrm{T}_{\mathrm{m}}\right)$ ) even though the same material would not exhibit any creep under bulk condition at the same temperature.

Nanoindentation creep has been observed in a wide range of materials including glasses, ceramics, and metallic materials, [124-126]. The indentation creep rate for high melting point materials can be of the order of $5 \times 10^{-5} \mathrm{~s}^{-1}$. This is a large value when compared to the corresponding bulk strain rate. The models for indentation creep are the same as those for bulk

\footnotetext{
${ }^{4}$ A major part of this work has been published in International Journal with Citation "Gan, M, and Tomar, V., 2010, Role of length scale and temperature in indentation induced creep behavior of polymer derived $\mathrm{Si}-\mathrm{C}-\mathrm{O}$ ceramics, Accepted to Appear in Materials Science and Engineering-A, August 06, 2010, Manuscript ID: MSEA-D-10-0176, DOI: 10.1016/j.msea.2010.08.016"
} 
creep, with the equivalent stress exponent, $n$, used as an indicator of the creep mechanism, [126, 127]. It is believed that when the value of $n$ is 1 , creep is controlled by vacancy diffusion as deformation mechanism, $[128,129]$; when the $n$ value is 2 , the creep mechanism the controlled by grain boundary sliding, [130]; when $n$ is 3 , diffusion-controlled dislocation motion dominates as deformation mechanism, $[131,132]$; and when $n$ is 5 , it is dislocation climb-controlled creep mechanism, [133]. During indentation creep tests on certain metals, alloys, and ceramics at room temperature, high $n$ values up to hundreds have been observed, [134-140]. The mechanism behind such high stress exponent values has been attributed to volumetric densification and dislocation pile up. Materials systems analyzed include metallic thin films, e.g. $\mathrm{Cu}, \mathrm{Al}, \mathrm{Ni},[141-$ 143], thin films made of metallic alloys, e.g. $\mathrm{Ni}_{3} \mathrm{Al}$, [144], and ceramics e.g. $\mathrm{ZrO} 2$, [145], and SiC-based, [146-148]. During such studies, the size of the indenter (owing to indentation size effect-ISE) and the grain size of the polycrystalline materials have been shown to strongly affect the indentation creep response of materials. Besides the ISE and the grain size effect, the creep deformation mechanism is also shown to be affected by the test temperatures and the change in microstructure of the material as a result of temperature change. It has also been shown that fiber reinforcement, [149], or particle reinforcement, [150], can constrain creep rate.

This work for the first time presents nanoindentation and microindentation creep analyses on polymer derived $\mathrm{Si}-\mathrm{C}-\mathrm{O}$ ceramic coatings at temperatures ranging from room temperature to 500 degree-C. The Si-C-O ceramic coatings were made using polymer-precursor processing. This method is a novel way to process low dimensional ceramics such as fibers and coatings, sometimes collectively referred to as the polymer-derived ceramics (PDCs). Due to high thermal resistance, excellent mechanical strength under elevated temperatures, Si-C-O based PDCs can be used in ceramic heaters, thermal barrier coatings, wear protections coatings, or micro electro 
mechanical devices. For example, it is used in the steam methane reforming (SMR) reaction chambers, where the steel is working under 800 degree-C in the atmosphere of steam and methane, [151]. In PDCs, the microstructure and subsequent properties can be adjusted by adding filler particles. The filler particles can be inert, e.g. $\mathrm{SiC}$, or active, e.g. $\mathrm{TiSi}_{2}, \mathrm{CrSi}_{2}, \mathrm{ZrSi}_{2}$, TiAl, and FeAl. Low creep rate is an essential property of this material system. Grain boundaries (GBs) are not present in this material system. Possible length scale related factors influencing creep rate include phase transformation, change in porosity etc. Nano- and micro scale investigations of creep deformation can provide important insights into achieving control of creep in this material system. While nanoindentation can give information about features such as individual particles, microindentation can reveal information about the mechanical behavior of collective microstructural features over a larger area.

\section{§2. METHODS}

The Si-C-O ceramic coatings investigated in this research were made using processing methods based on polymer-precursors by Prof. Raj Bordia's group at the University of Washington, Seattle-WA, [152-155]. The coating thickness is approximately $15 \mu \mathrm{m}$. All tests were performed using a multi-module mechanical tester (Micro Materials Ltd, UK) shown in Fig. 1. Figure 1 (b) illustrates the setup for performing high temperature indentation. Both the sample and the indenter tip were heated to the required testing temperature which was closely monitored using thermocouples. The remaining parts of setup, which have to be maintained at room temperature, are separated using heat shields. The tests were performed at 6 different temperatures: room temperature, 100 degree-C, 200 degree-C, 300 degree-C, 400 degree-C, and 500 degree-C. 


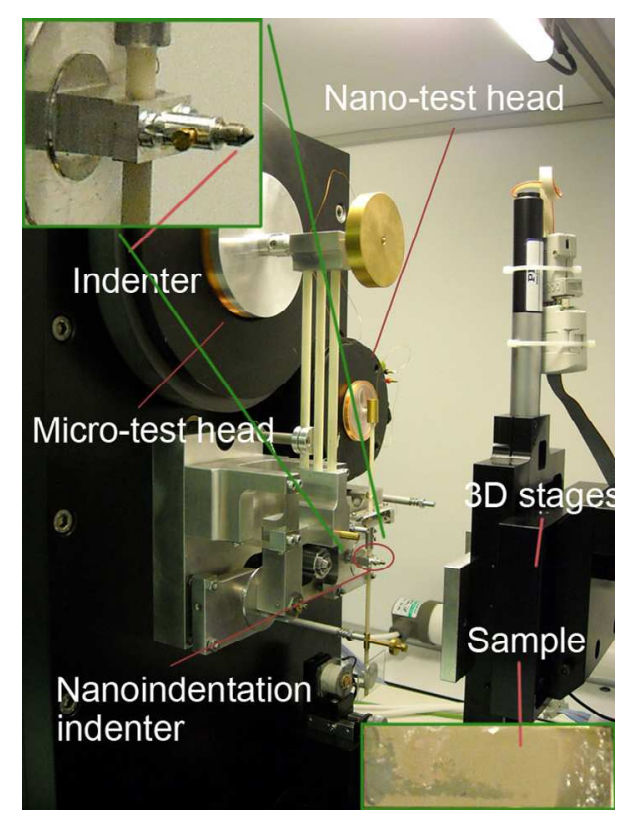

(a)

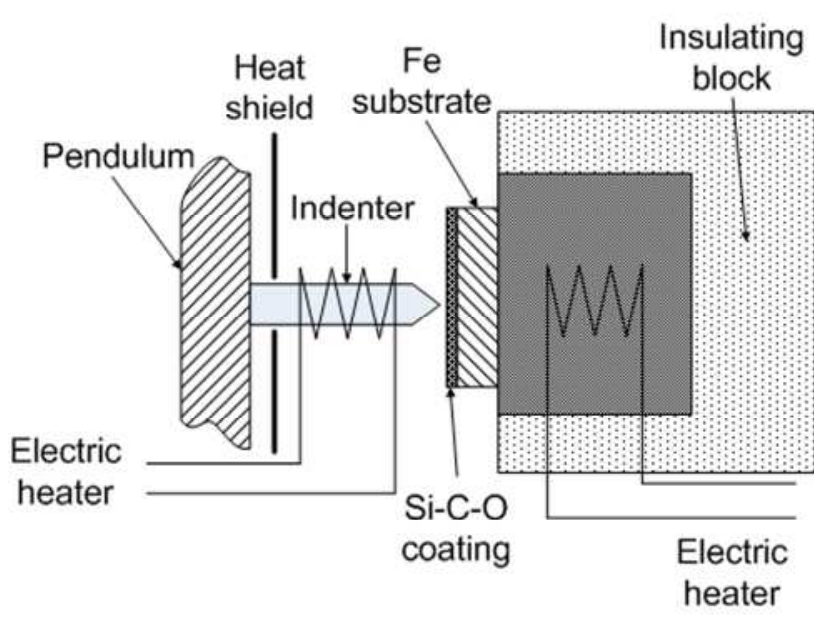

(b)

Fig. 1: Experimental setup. (a) Picture showing the setup of nano- and microscale testing heads and (b) schematic showing the high temperature indentation approach

The tip radius of nanoindenter is approximately $20 \mathrm{~nm}$ and of microindenter is approximately $200 \mathrm{~nm}$. The projected area of the nanoindenter is approximately $0.5 \mu \mathrm{m}^{2}$ and of the microindenter is approximately $5 \mu \mathrm{m}^{2}$. Both indenters are of Berkovich type. During the tests, the samples were mounted on the indentation stage using glue. The indenter approached the sample in its thickness direction, Fig. 1(b). Indentation locations were selected randomly on sample surfaces. In the case of each chosen location, tests were performed in either a 3 by 3 matrix or in a 4 by 4 matrix pattern with equal longitudinal and transverse spacing between each indentation spot of approximately $5 \mu \mathrm{m}$ in the case of nanoindentation and $20 \mu \mathrm{m}$ in the case of microindentation. The indentation depths and maximum indentation load were chosen to minimize the effect of measurement drift that usually occurs in indentation tests if the depth is too small. The nanoindentation depths were in the range of $200 \mathrm{~nm}$ to $500 \mathrm{~nm}$ and the 
microindentation depths were in the range of 1 to $3 \mu \mathrm{m}$. At such depths the effect of tip radius as well as substrate is negligible.

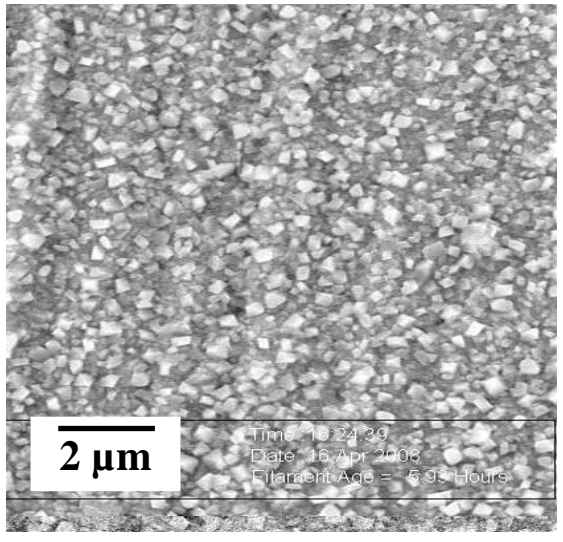

(a)

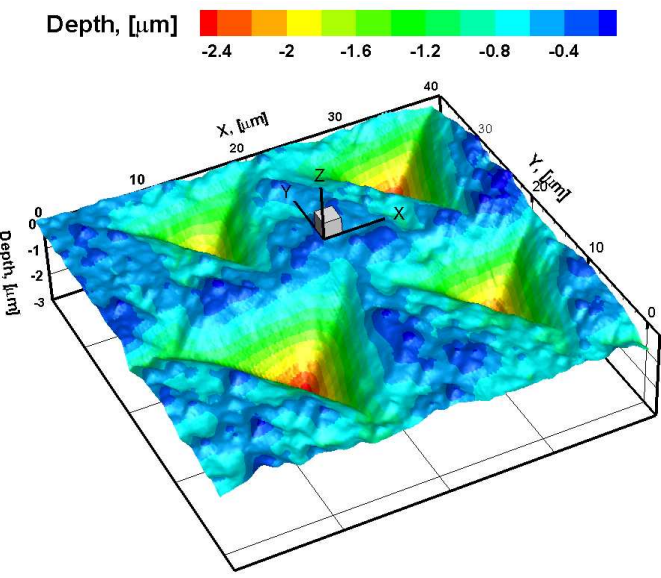

(b)

Fig. 2: (a) SEM images of Si-C-O ceramic coatings and (b) image showing a comparison of the indentation depth with the Si-O-C coating surface

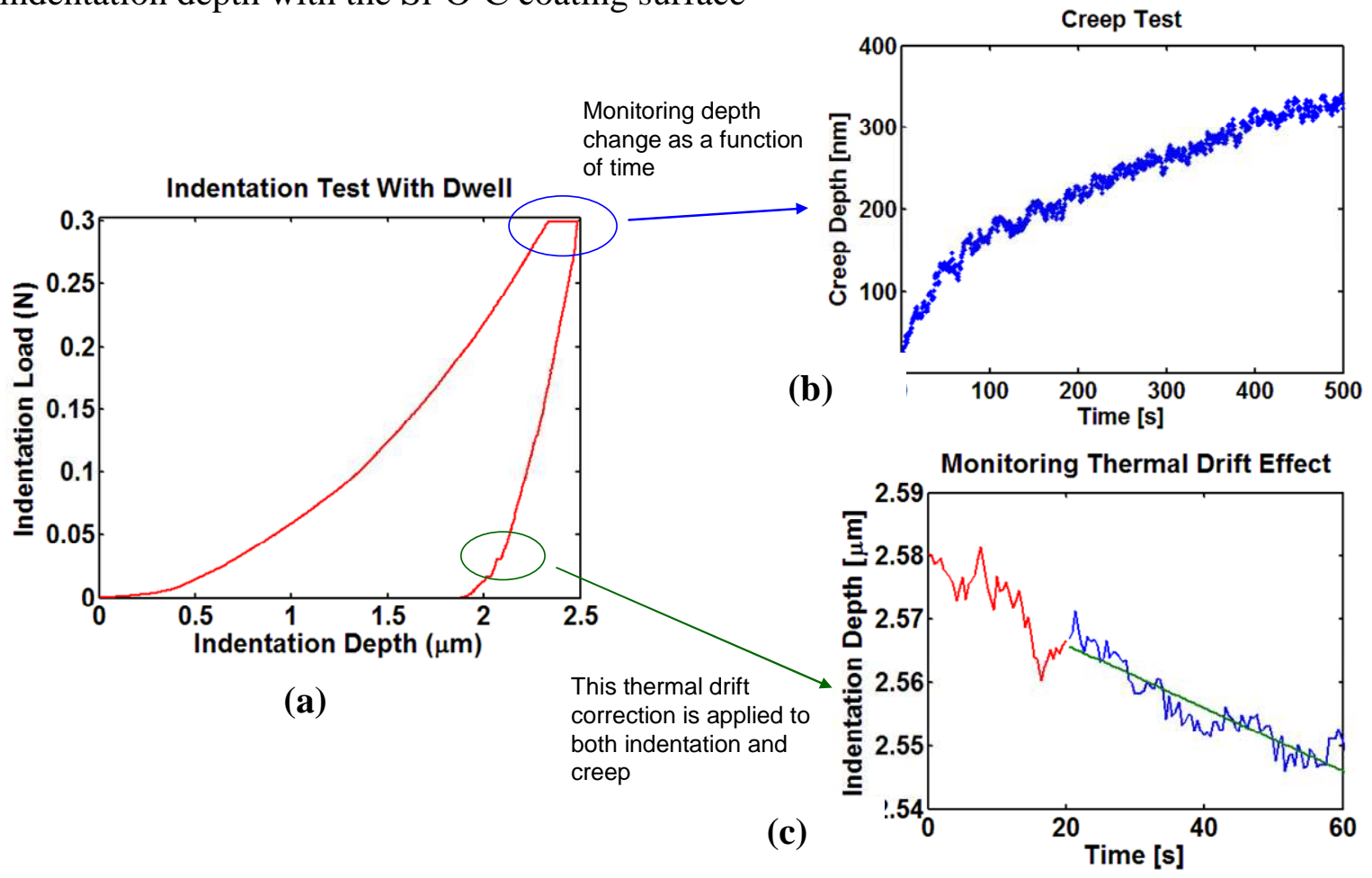

Fig. 3: (a) A typical nanoindentation profile showing (b) how the creep data is extracted and (c) how the thermal drift rate is extracted 
Figure 2 also shows a surface scan of a coating after microindentation test at the peak indentation load of $750 \mathrm{mN}$. As shown, the microindentation depth is significantly larger than the size of surface asperities. Therefore, the surface roughness does not affect the microindentation test results. Since the length scale of nanoindentation tests is of the order of the size of particles, the surface roughness also does not affect nanoindentation results. Before starting the indentation tests, efforts were made to minimize the effects of thermal drift by allowing thermal equilibrium to be reached. This was ensured by setting the experimental setup idle for 2 hours at each temperature before each indentation testing run. The whole testing system was kept in a chamber to keep the temperature constant, which was set to be 3 degrees above the environmental temperature. Berkovich diamond indenter was used in this experiment, for which the contact area as a function of indenting depth was calibrated using fused silica and Tungsten reference samples at room temperature. Figure 3 shows an example of the procedure used to extract the indentation creep data. The creep deformation profile is extracted from the dwell period at the peak load, Fig. 3(b). Dwell period may affect the creep data. A number of dwell periods were tried. A dwell period of $500 \mathrm{~s}$ was decided based on convergence in the measurements. Indentation profiles were imaged before and after the tests to ensure that similar surface conditions exist before and after the tests. At each load, more than nine independent indentations were performed.

One major concern is the stability of the indentation equipment as measurement temperature is increased. As a result of changes in stability, the measured depth as a function of time may drift from the actual value. Such a drift is sometimes referred to as thermal drift. During experiments, care is taken to minimize thermal drift by allowing thermal equilibrium to be reached. In addition, thermal drift rate is measured during each indentation test to adjust the measured data. In order to measure thermal drift rate for each indentation run, the indentation 
load was held constant for a period of 100 s typically at $10 \%$ of the peak during unloading, Fig. 3(c). Figure 4 shows the averages of measured thermal drift rates as a function of peak indentation load and temperatures during nanoindentation and microindentation tests. As shown, thermal drift rate is a strong function of the peak indentation load at both length scales. The thermal drift rate increases with increase in load and temperature at both scales. The magnitude of thermal drift rate is higher at the nanoscale owing to much higher sensitivity of measurements at the nanoscale. During calculation of creep data, the thermal drift rate is multiplied by the dwell time and then the result is subtracted from the measurement.

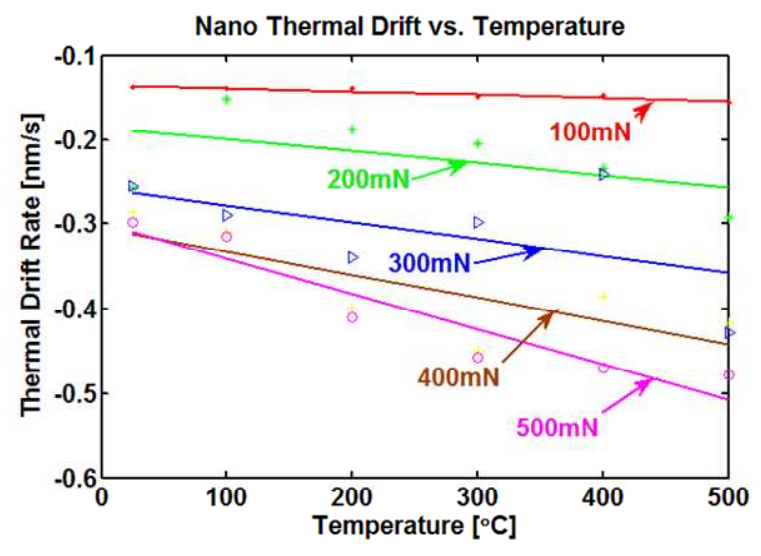

(a)

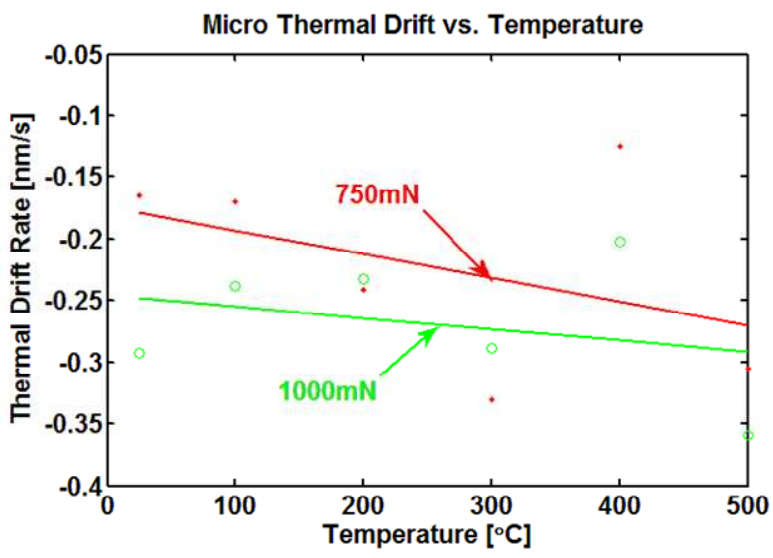

(b)

Fig. 4: Thermal drift rate as a function of temperature and the peak indentation load in the case of (a) nanoindentation and (b) microindentation.

\section{§2.1 Indentation and Creep Measurement Procedure}

In the case of metals and metallic alloys, plastic deformation under indentation and the nanoindentation creep deformation are separated by dislocation pop-in events which are characterized by a horizontal constant load line on the $P$ - $h$ (load-depth) curve. In this case, holding the load above the pop-in value for any time and just below the pop-in value for very long holding time will lead to indentation creep. In the case of ceramics, e.g. quartz, usually a 
distinctive pop-in event is not observed on increasing indentation load, (an exception to this trend has recently been reported by Guicciardi [156]). Therefore, the creep investigations have to be performed at multiple peak indentation loads. This is the approach adopted in the present work. The modulus and hardness were calculated using the well known Oliver-Pharr method, $[157,158]$. A typical indentation unloading curve shown in Fig. 3 (a) can be described by the Oliver-Pharr method as:

$$
P=A\left(h-h^{\text {final }}\right)^{m}
$$

In the above Eqn., $A$ and $m$ are material constants. Parameter $h_{\text {final }}$ is the indentation depth after complete unloading. The contact depth $h_{c}$ at the peak load $P_{\max }$ which corresponds to the total indentation depth $h_{\max }$ are related as

$$
h_{c}=\varepsilon \frac{P_{\max }}{S} .
$$

Here, $\mathrm{S}$ is the stiffness. In this case, stiffness is the initial unloading stiffness, $S_{P \max }$. The parameter $\varepsilon$ is a correction factor; $\varepsilon=1$ for a spherical tip and $\varepsilon=0.75$ for a Berkovich tip. The area of contact is calculated as a function of contact depth for Berkovich indenter as:

$$
A_{c}=3 \sqrt{3 h_{c}^{2}} \tan ^{2} 65.3=24.5 h_{c}^{2}
$$

The reduced modulus, $E_{r}$, is given by

$$
\frac{1}{E_{r}}=\frac{1-v_{i}^{2}}{E_{i}}+\frac{1-v_{s}^{2}}{E_{s}} .
$$

Here $v$ is the Poisson's ratio, $E$ is the elastic modulus. Subscripts $i$ and $s$ represent the indenter tip and specimen (for the diamond tip, $v_{i}=0.07$ and $E_{i}=1141 \mathrm{GPa}$ ), respectively. The stiffness $S$ is related to the load, depth, contact area, and reduced modulus by the following relation:

$$
S=\frac{d P}{d h}=2 E_{r} \sqrt{\frac{A_{c}}{\pi}} .
$$


Hardness $\mathrm{H}$ is given as:

$$
H=\frac{P_{\max }}{24.5 h_{c}^{2}} .
$$

The elastic modulus of the sample $E_{s}$ can be calculated from the equation give by:

$$
E_{s}=\frac{d P}{d h} \frac{1}{2 h_{c}} \frac{1}{\beta} \sqrt{\frac{\pi}{24.5}}
$$

In the above equation, $\beta$ is a tip parameter which is equal to 1.034 for a Berkovich indenter tip. In depth-sensing indentation with a self-similar indenter, the strain-rate, $\dot{\varepsilon}$, stress, $\sigma$, and instantaneous indentation depth, $h$, are related as,

$$
\dot{\varepsilon} \sim \frac{\dot{h}}{h} \quad \text { and } \quad \sigma \alpha \frac{P}{h^{2}}
$$

In the above Eqn., $P$ is indentation load and $\dot{h}=d h / d t$, where $t$ is time. The scaling relations in Eq. (38) are based on the analyses for a power law creeping solid, [159]. The relations assume that in the case of indentation by conical punch the ratio of $h$ and radius of contact zone $(a)$ depends only on stress exponent $(n)$. A value of $n=1$ implies exact linear viscoelastic material behavior which leads to $\mathrm{P} / \mathrm{h}^{2} \alpha \dot{h} / h$. Based on Eq. (38), three different choices are available to calculate creep stress exponent: (1) depth sensing constant load (h-CL) method, [160], (2) the continuous stiffness constant load (S-CL) method, [161], and (3) the exponential load (EL) method, [162]. The two constant load methods use data from a load hold at the maximum load. In our experiments the h-CL method is used to calculate stress exponent. Since the load is constant, the EL method cannot be used. Because of the small creep displacements, the small changes in stiffness cannot be separated from the noise background. Therefore, S-CL method is also not reliable. During indentation, because the contact area will increase during the holding period if creep occurs, the hardness will subsequently decrease with the corresponding decrease 
in the indentation strain rate. Tabulation of a series of pairs of the strain rate and stress based on the measured data is used to calculate stress exponent. For the scaling of strain rate and stress according to Eq. (38) to be correct, the indentation tip has to be self-similar. In order to ensure this, the indentation depth must not be influenced by the tip head curvature. In order to ensure this, the tip depth was maintained to be more than $500 \mathrm{~nm}$ in the case of nanoindentation and 1 $\mu \mathrm{m}$ in the case of microindentation. Both of these values are more than 10 times the tip radius.

Evaluation of plastic deformation parameters from indentation experiments is a difficult problem. Usually, yield stress for ceramics under nanoindentation is related to the deviation from elastic behavior in spherical indentation experiments, where relations for transforming indentation stress and strain into equivalent uniaxial stress and strain quantities are available, [163]. Based on indentation experiments on metals, Tabor [164] defined the uniaxial flow stress $\sigma_{0}$ for sharp indentation in terms of hardness $\mathrm{H}$ as: $\mathrm{H}=3 \sigma_{0} \approx 3 \sigma_{\mathrm{Y}}$. Here, $3 \sigma_{0}$ measured stress for a Vickers indenter at a representative strain $\left(3 \varepsilon_{0}\right)$ of $0.08-0.1$. Finite element analyses have revealed that the representative strain lies between 0.25 and 0.36 . However, Cheng and Cheng [165] have shown that that $\sigma_{y} \approx H / 3$ may just be a first approximation. Janakiraman and Aldinger [147] have shown that the yield stress multiplying factor varied between 1.7 and 2 for polymer derived $\mathrm{SiCN}$ ceramics. Since yield stress and hardness are correlated by a multiplying factor in almost all cases, the yield stress calculations are not performed in this work. Instead the trend is extracted from the hardness data.

During analyses, the temperature dependent (from room temperature upto $500{ }^{\circ} \mathrm{C}$ ) nanoscale and microscale elastic moduli of the Si-C-O coating were first measured. The maximum load in the nanoindentation creep tests varied from $100 \mathrm{mN}$ to $500 \mathrm{mN}$, with an increment of $100 \mathrm{mN}$. For the micro-indentation creep tests, the maximum loads were $750 \mathrm{mN}$ 
and $1000 \mathrm{mN}$. Load and depth calibration were performed before each experiment for better precision of the measurement.

\section{§2.2 Data Analyses Procedure}

The derivation of the strain rate requires differentiation of the $h(t)$ curve. As shown in Fig. 5, the $h(t)$ data is scattered around a trend line.

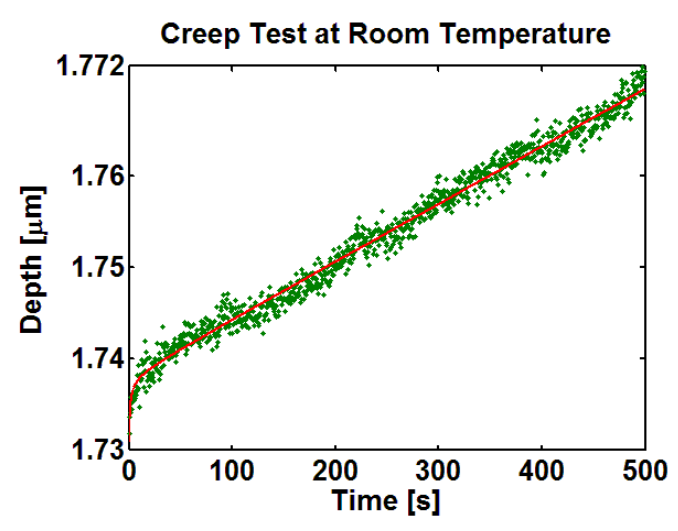

(a)

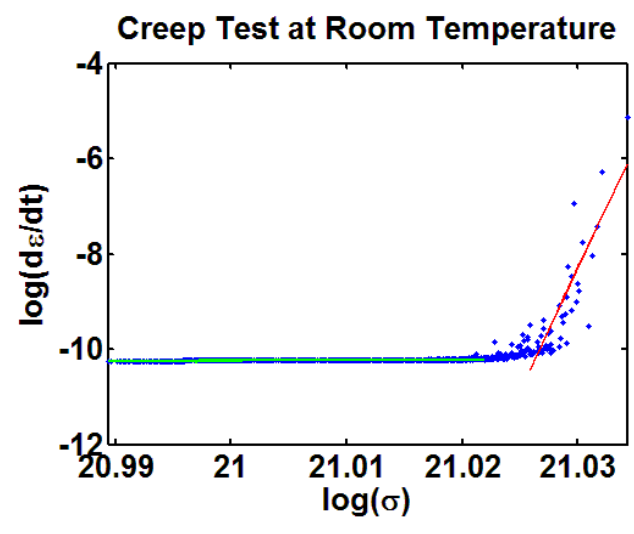

(b)

Fig. 5: Illustration of stress exponent calculation. (a) The creep raw data file was plotted and fitted using Eqn. (10); and (b) The equivalent strain rate and stress based on the fitted data. The horizontal line is used to calculate steady state stress exponent.

A commonly used fitting function for $h(t)$ developed by Li and Ngan [135] is given a

$$
h(t)=h_{i}+a\left(t+t_{i}\right)^{b}+k t .
$$

The above equation was used by Ma et al [166] to investigate the creep behavior of thin $\mathrm{Ni}$ film and by Cao et al [137] in investigating the creep behavior of thin Ta film. A slightly modified form (Eqn. (40)) was used in our analysis for less fitting errors and better convergence.

$$
h(t)=h_{i}+a t^{b}+k t .
$$


Using the $h(t)$ curve, the relations in earlier section are used to calculate the stress exponent and creep strain rate. In the subsequent section, the elastic moduli, stress exponent trend, and creep rate (strain rate) are investigated as a function of temperature, length scale, and maximum indentation load.
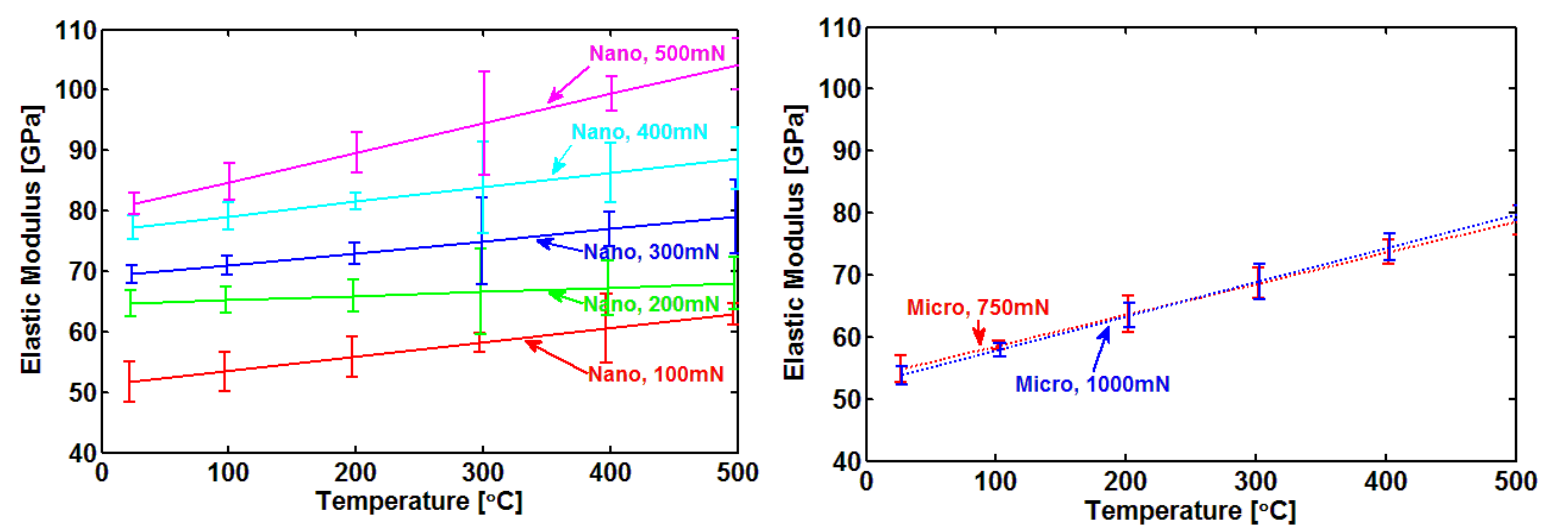

Fig. 6: (a) Nanoindentation elastic moduli and (b) microindentation ela coatings as a function of temperature and maximum indentation load

\section{§3. RESULTS}

Figure 6 shows the elastic moduli values as a function of testing temperature and peak indentation load. As shown, the elastic moduli increase with increase in temperature and with increase in peak load at the nanoscale. At the microscale the elastic moduli increase as a function of temperature. However, dependence on the peak load at the microscale is not clear. Increase as a function of peak load signifies the ISE. It has been shown that Young's modulus of $\mathrm{SiO}_{2}$ will increase with temperature due to compaction and distortion of $\mathrm{SiO} 4$ tetrahedra in the temperature range of experiments. The underlying SiCO matrix retains this attribute. An examination of Fig. 6 (a) reveals the ISE is stronger at higher temperatures. The ISE is absent in the case of microscale data. The reason behind the observation of ISE at the nanoscale is not clear. For each data point in Fig. 6(a), a minimum of 16 tests were performed. Therefore a possibility that a 
single microstructural aberration is contributing to the ISE is ruled out. At the nanoscale the nanoindenter radius is approximately $20 \mathrm{~nm}$ and projected area is approximately $0.5 \mu \mathrm{m}^{2}$. Accordingly, the indenter samples either the SiC particles or small spaces of SiCO matrix between adjacent $\mathrm{TiSi}_{2}$ particles. The ISE, therefore, can be attributed to increasing nanoscale densification with increasing indentation depth. At the microscale collective deformation of a sample volume containing many $\mathrm{SiC}$ particles takes place. Due to such collective motion of particles under indenter tip, the effect of volumetric densification under indenter tip is minimzed leading to the absence of ISE. Increase in temperature contributes to increased binding of $\mathrm{TiSi}_{2^{-}}$ SiCO interfaces invariably leading to increase in the elastic moduli values as a function of temperature at both scales.

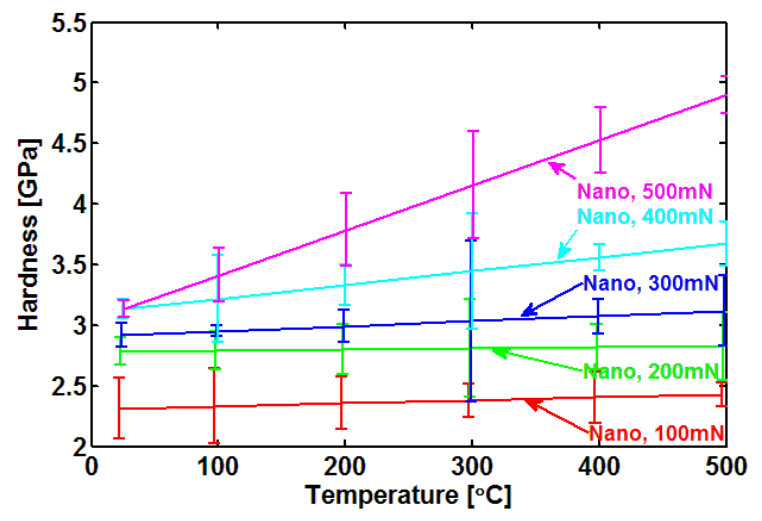

(a)

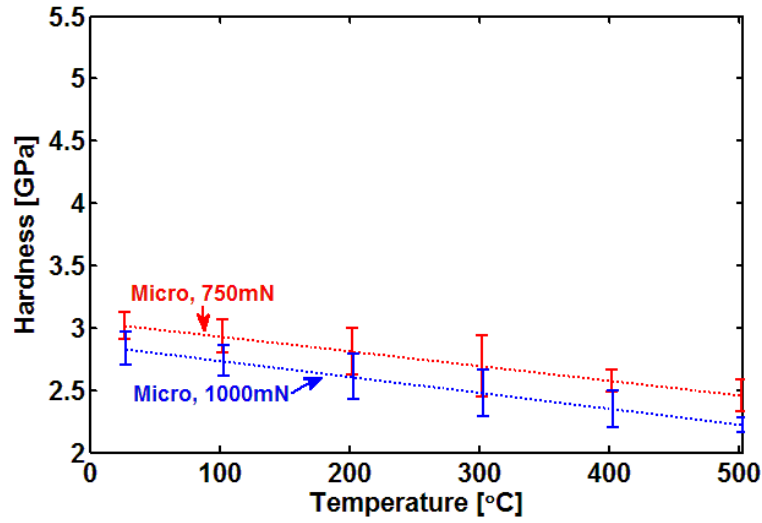

(b)

Fig. 7: (a) Nanoindentation hardness and (b) microindentation hardness of Si-C-O coatings as a function of temperature and maximum indentation load

Figure 7 plots the hardness data corresponding to the moduli plots in Fig. 6. As shown, at the nanoscale the trend for the elastic moduli is repeated for the hardness values. A clear trend on increase in Meyer's exponent with increase in temperature emerges from nanoindentation data. The microindentation hardness data trend is opposite to the trend shown in the case of microindentation elastic moduli. Hardness decreases as the measurement temperature increases. 
In addition, hardness reduces with increase in the peak indentation load, signifying strain softening of the material. Reduction of hardness with increase in temperature is attributable to stronger $\mathrm{TiSi}_{2}$ particles pressing in relatively softer $\mathrm{SiCO}$ matrix getting progressively softer with increase in temperature at the microscale. At the nanoscale not enough contact area is available to have such an effect. Data on correlation between temperature dependent hardness and young's moduli values for ceramics is not available in the literature. A similar trend (increase in moduli and decrease in hardness as a function of temperature) has been observed for Chromium steels by Medved and Bryukhanov [167].

It has been shown that in Si-C-O PDCs in amorphous phase the deformation during indentation occurs due to volumetric densification events. It has been shown that volumetric densification promotes strain hardening in vitreous silica under both indentation and diamondanvil compression experiments, [148]. The microindentation experiments on the present materials in the amorphous state reveal a reduction of indentation hardness under increasing applied loads, indicating densification-assisted strain softening of these materials, [148]. A combination of nanoscale and microscale results points that individually neither the $\mathrm{SiCO}$ matrix nor $\mathrm{TiSi}_{2}$ particles show strain softening. However, mechanistic effects related to their combined presence at the microscale results in strain softening behavior. Cheng and Cheng, [168], have pointed out that indentation hardness is not a function of indentation depth for materials that truly follow power-law strain hardening. This is in contrast to the nanoindentation and microindentation results from the present materials that show an increase in hardness with increasing load during nanoindentation and reduction in hardness with increasing load during microindentation. Apart from the influence of strain hardening or softening, material pile-up 
around the indent and indentation creep can also contribute to indentation hardness behavior observed in the present work.

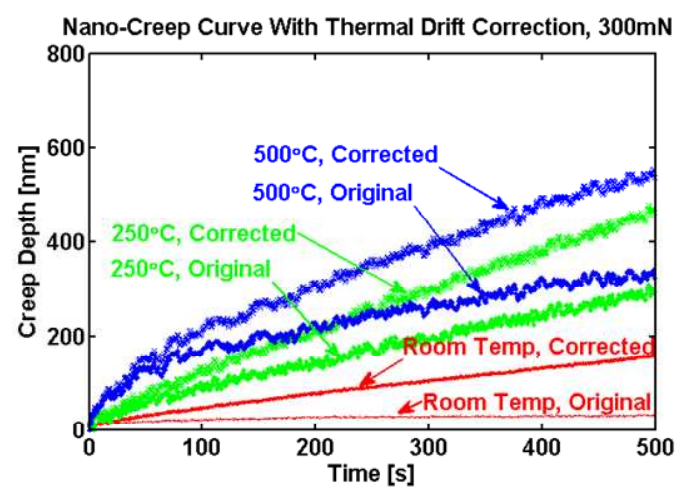

(a)

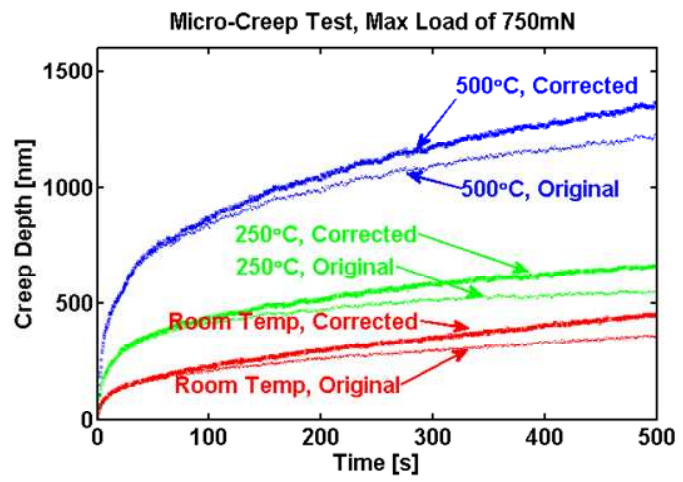

(c)

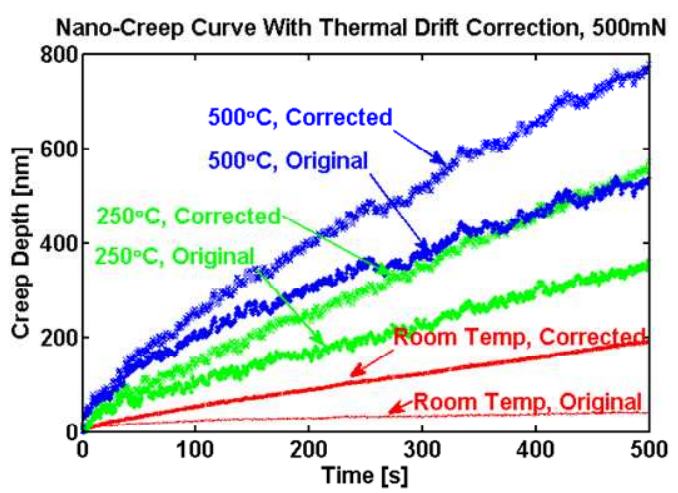

(b)

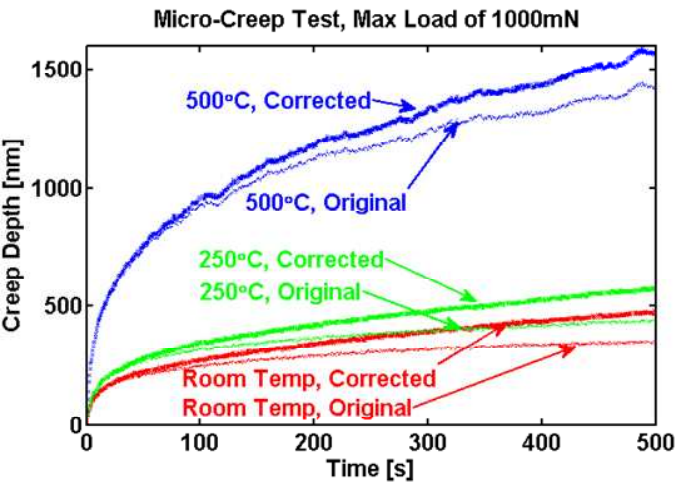

(d)

Fig. 8: Indentation depth (original and thermal drift corrected) as a function of time at different temperatures in the case of (a) nanoindentation test at the peak load of $300 \mathrm{mN}$, (b) nanoindentation test at the peak load of $500 \mathrm{mN}$, (c) microindentation test at the peak load of 750 $\mathrm{mN}$, and (d) microindentation test at the peak load of $1000 \mathrm{mN}$

Figure 8 plots creep depth as a function of dwell period at three different temperatures at two different nanoindentation and microindentation peak loads. Creep data is normalized by subtracting the initial depth of each creep test. For comparison, the plots also show the thermal drift uncorrected as well as corrected data. As expected, the effect of thermal drift correction is 
the highest at the highest temperature and at the nanoscale. The creep depth vs. time plots reached steady state within first $100 \mathrm{~s}$ of plotting in all cases. Effect of temperature is to increase the creep rate. The temperature effect is more pronounced at the micro scale in comparison to the nanoscale. The trend is particularly strong when transitioning from $250{ }^{\circ} \mathrm{C}$ to $500{ }^{\circ} \mathrm{C}$.

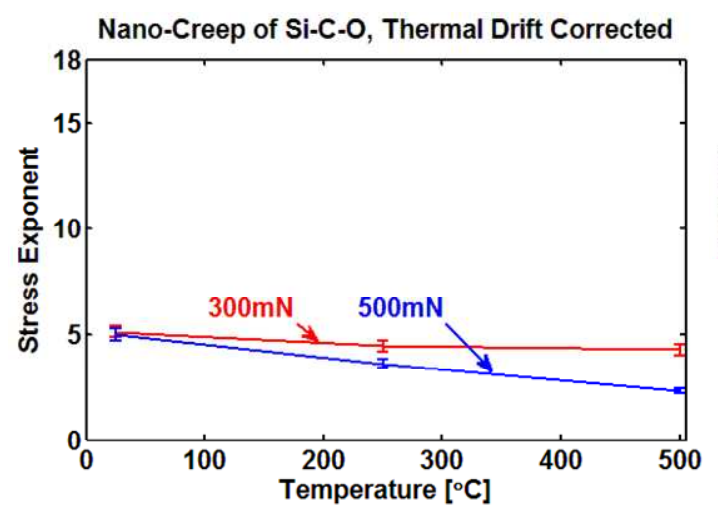

(a)

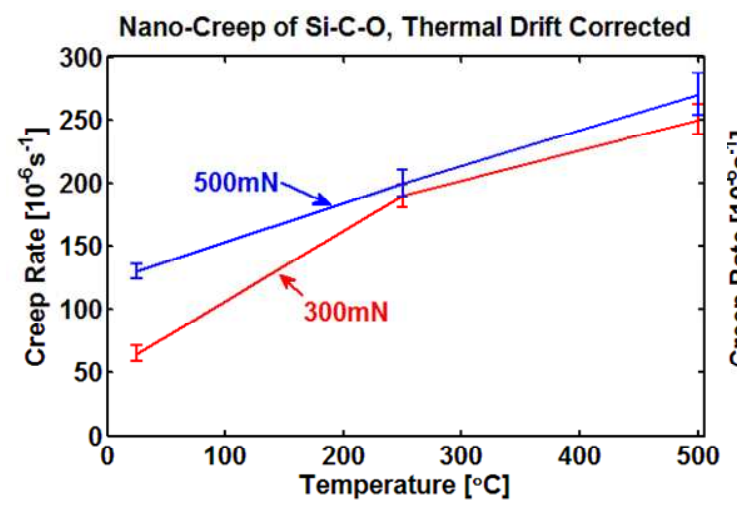

(c)

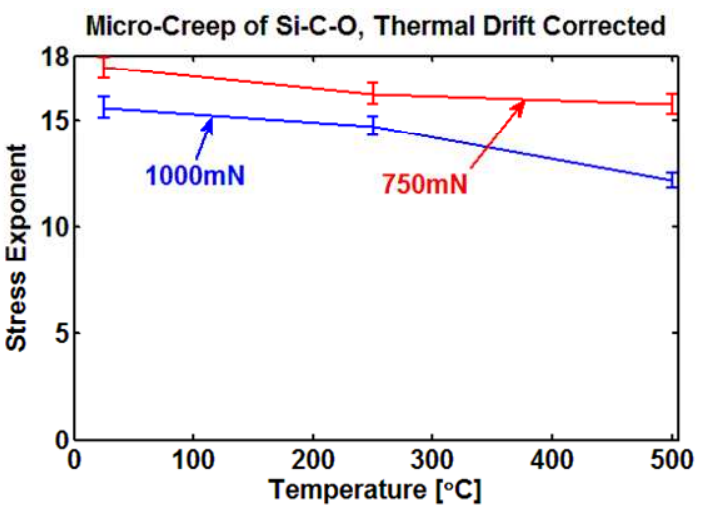

(b)

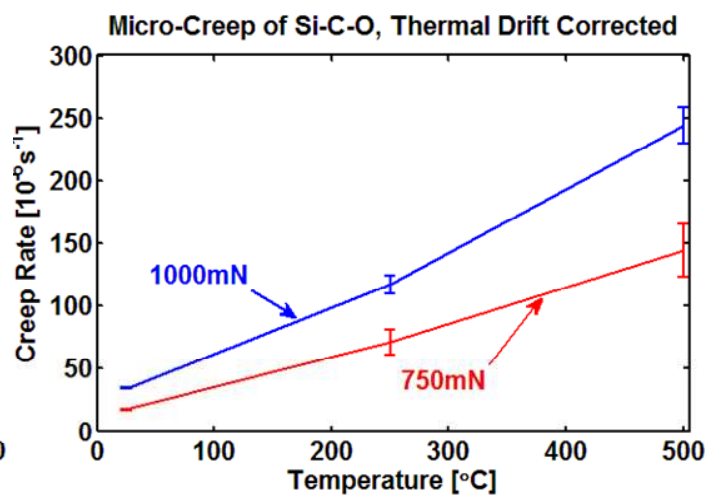

(d)

Fig. 9: (a) stress exponent as a function of load during nanoindentation creep measurements, (b) stress exponent as a function of load during microindentation creep measurements, (c) creep strain rate as a function of load during nanoindentation creep measurements and (d) creep strain rate as a function of load during microindentation creep measurements

Figure 9 plots stress exponent and creep strain rate as a function of temperature and peak indentation load at both length scales. Stress exponent at the nanoscale lies in the range of 4 to 5 indicating a dislocation climb related creep deformation mechanism occurring through bulk or 
pipeline diffusion of dislocations. The exponent reduces with increase in temperature indicating a transition of mechanism from dislocation climb to diffusion. The densely dislocated structure with high dislocation density under indenter may result in dense dislocation channels directing atoms from dislocated cores to free surfaces resulting in such a mechanism occurring at low homologous temperatures. At the microscale the stress exponent is considerably higher indicating that primary mechanism of deformation is volumetric densification. Such a transition suggests a rapid change of mechanism from linear diffusion flow to power law mechanism (e.g. climb) and eventually to rate insensitive plastic flow (dislocation glide) as the indentation size moves towards microscale regime. The disparity in stress exponent values at the two length scales is not reflected in the creep strain rate data. As shown, the creep strain rates at both length scales lie in the same range with microscale data being on the lower side. Creep strain rate generally increases as a function of increase in temperature as well as with increase in peak indentation load. The creep strain rate at the microscale being lower than that at the nanoscale can be attributed to the averaging effect of measurements. As discussed earlier, in the case of microscale measurements, the indenter covers an area that is approximately 10 times larger than the nanoindenter area. Since hardness reduces and creep strain rate increases with increase in temperature at the microscale, the strain rate sensitivity index (constant $\mathrm{k}$ in relation $\sigma=\mathrm{b} \dot{\varepsilon}^{\mathrm{k}}$ ) indicates strain softening at the microscale. The same analyses reveal strain hardening at the nanoscale. 


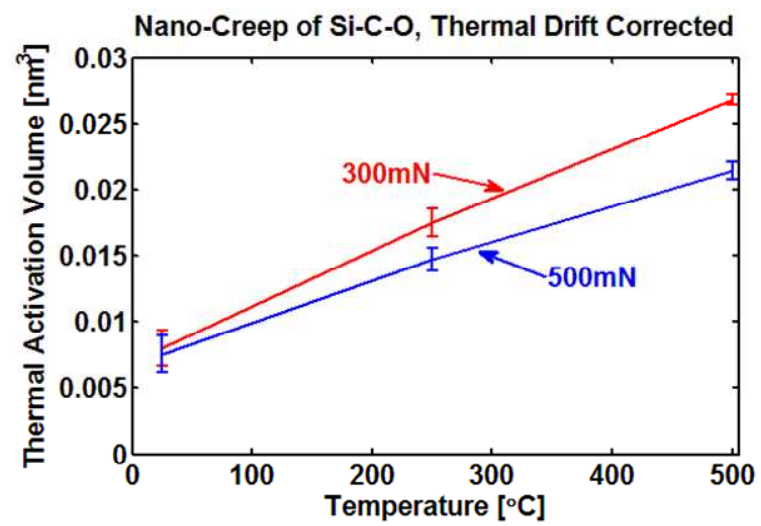

(a)

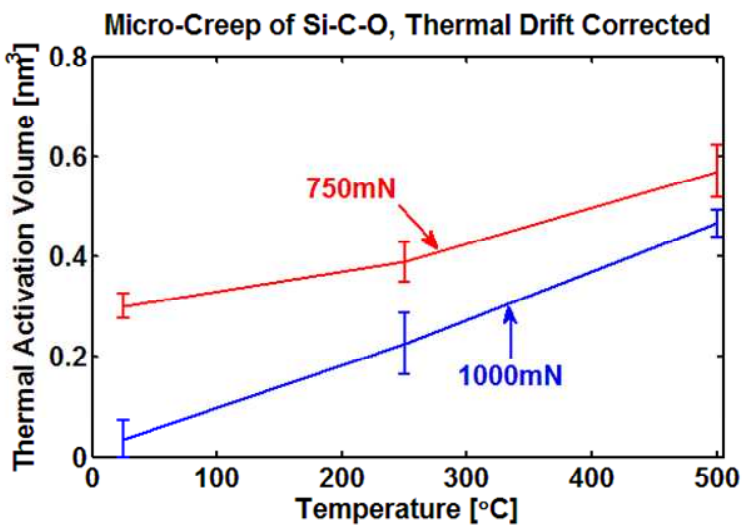

(b)

Fig. 10: Thermal activation volume as a function of peak indentation load and measurement temperature at (a) nanoscale and (b) microscale.

\section{§4. DISCUSSIONS}

In general, in the case of PDCs both dislocation dominated and volumetric densifying type of deformation mechanisms with varying degree are present during indentation. In the case of quartz, the size effect on the stress exponent can be explained by a reduction of the localized shear volume as the indent size decreases. However, in the present work the size effect is related to shift from dislocation climb and diffusion related mechanism at the nanoscale to volumetric densification at the microscale (Fig. 9). The extent of a particular type of mechanism determining deformation is governed by the availability of excess free volume which is related to thermal activation volume, which is also the average change in volume of the flow unit. Low-temperature creep deformation strain rate, $\dot{\varepsilon}$, of materials can be expressed by Arrhenius-type flow function, [169],

$$
\dot{\varepsilon}=\dot{\varepsilon}_{o} \exp \left[-\frac{\Delta G(\sigma)}{k_{B} T}\right]
$$

In the above Eqn., $\dot{\varepsilon}_{o}$ is reference strain rate that varies with the type of material, stress level, and microstructure, $\Delta G$ is the activation energy for creep or other rate-dependent process, $k_{B}$ is 
Boltzmann's constant and $T$ is temperature in Kelvin. Parameter $\dot{\varepsilon}_{o}$ is proportional to the concentration of elementary defects which cause plastic strain, [170]. The sensitivity of strain rate to stress is mainly determined by the $\Delta G$ term. The thermal activation volume $\left(V^{*}\right)$ is expressed by the partial derivative of $\Delta G$ with respect to stress $\sigma$, [171]. In the present work, $V *$ is approximated as

$$
\left.V^{*} \equiv k_{B} T \frac{\partial \ln \dot{\varepsilon}}{\partial \sigma}\right|_{\varepsilon, T}
$$

The derivatives are taken at fixed strain at each temperature to constrain the influence of structure change due to work hardening and softening. Figure 10 plots the thermal activation volume calculated in this case as a function of temperature, peak indentation load, and length scale. As shown, thermal activation volume increases with increase in the length scale of measurements. Since, the thermal activation volume is directly correlated to the deformation energy, the trend implies a significantly high deformation work required at the microscale in comparison to the nanoscale. An almost linear increase in activation volume with increase in temperature implies that deformation mechanism is primarily controlled by dislocations. At the nanoscale the thermal activation volume is 10 times less than that at the microscale indicating that the dislocation pile up is restricted as well as that dislocation climb and diffusion is the main deformation mechanism. At microscale significantly higher thermal activation volume indicates availability of excess free volume in the material structure that can be eliminated by densification. A combination of earlier findings with this result indicates that with increase in length scale the deformation mechanism switches from dislocation diffusion to dislocation pileup. With increase in length scale, dislocation motion does not face significant obstacles or phase transformation related factors. Such factors, if present, will change the almost linear trend observed in Fig. 10. 
The observed trend of the data on peak indentation load as well as stress exponent values at room temperature are in close agreement with the work of Li and Ngan [124]. However, stress exponents are lower than those reported by Li and Ngan for fused silica, indicating a difference in deformation mechanism. The deformation mechanism is intricately linked with the microstructure of this material. The material has $\mathrm{TiSi}_{2}$ particles with higher melting point embedded in SiCO matrix with lower melting point. With increase in temperature, softening of the matrix, particle sliding, and particle rearrangement contribute to deformation mechanism. The effect of these factors is more pronounced at microscale due to higher surface area sampled.

\section{§5. SUMMARY AND CONCLUSION}

In the present study, nanoindentation and microindentation mechanical behavior of Si-C-O ceramic coatings was investigated as a function of temperature. The properties of focus include elastic modulus, hardness, creep exponent, and creep strain rate. At the nanoscopic length scale the deformation mechanism is dominated by dislocation climb and diffusion. With increase in length scale the thermal activation volume increases by approximately 10 times. The excessive free volume leads to the deformation mechanism switching to volumetric densification and dislocation pile up. Another important physical effect analyzed is the effect of increase in temperature on the observed deformation mechanism. At the nanoscale, with increase in temperature, both hardness and elastic moduli show an increase. At the microscale, however, hardness reduces with increase in temperature. The ISE is observed at both length scales. However, at the nanoscale the ISE is linked with strain hardening. At the microscale, strain softening behavior is observed. Mechanistically, the plastic deformation behavior reveals that both the $\mathrm{SiCO}$ matrix and $\mathrm{TiSi}_{2}$ particles have strain hardening behavior. However, as a consequence of the shear effect caused by densification of $\mathrm{TiSi}_{2}$ particles under microindenter, 
the strain softening behavior is observed. Creep investigation reveal that length scale affects the stress exponent value. However, creep strain rate at both length scales is in the same range.

Overall, analyses reveal that both indentation creep and capability of strain hardening determine the length scale dependent indentation behavior in the material system. The effect of temperature is to introduce strain hardening at the nanoscale and strain softening at the microscale. 


\section{VI-REFERENCES}

[1] Weimer, A.W. and Bordia, R.K., Processing and properties of nanophase $\mathrm{SiC} \mathrm{Si}_{3} \mathrm{~N}_{4}$ composites. Composites Part B: Engg, 1999. 30: p. 647-655.

[2] Ajayan, P.M., Schadler, L.S., and Braun, P.V., Nanocomposite Science and Technology 2003: Wiley-VCH.

[3] Niihara, K., New design concept for structural ceramics-Ceamic nanocomposites. J. Ceram. Soc. Jpn: The centennial memorial issue, 1991. 99(10): p. 974-982.

[4] Ohji, T., Jeong, Y.-K., Choa, Y.-H., and Niihara, K., Strengthening and toughnening mechanisms of ceramic nanocomposites. J. Am. Ceram. Soc., 1998. 81(6): p. 1453-1460.

[5] Rendtel, A., Hubner, H., Herrman, M., and Schubert, C., Silicon nitride/silicon carbide nanocomposite materials: II, hot strength, creep, and oxidation resistance J. Am. Ceram. Soc., 1998. 81(5): p. 1109-1120.

[6] Niihara, K., New design concept for structural ceramics-Ceramic nanocomposites. J. Ceram. Soc. Jpn: The centennial memorial issue, 1991. 99(10): p. 974-982.

[7] Sternitzke, M., Review: Structural ceramic nanocomposites. J. Eur. Ceram. Soc., 1997. 17: p. 1061-1082.

[8] Tian, Y. and Shin, Y.C., Multiscale finite element modeling of silicon nitride ceramics undergoing laser-assisted machining. J. Manuf. Sci. and Engg., 2007. 129(287-295).

[9] Raiser, G.F., Wise, J.L., Clifton, R.J., Grady, D.E., and Cox, D.E., Plate impact response of ceramics and glasses. J. Appl. Phys., 1994. 75(8): p. 3862-3869.

[10] Krstic, V.D., Grain size dependence of fracture strength in anisotropic brittle solids. J. Mater. Sci., 1988. 23: p. 259-266.

[11] Zavattieri, P.D. and Espinosa, H.D., Grain level analysis of crack initiation and propagation in brittle materials. Acta Materialia, 2001. 49: p. 4291-4311.

[12] Zavattieri, P.D., Raghuram, P.V., and Espinosa, H.D., A computational model of ceramic microstructures subjected to multi-axial dynamic loading. J. Mech. Phys. Solids, 2001. 49: p. 27-68.

[13] Sands, C.M., Henderson, R.J., and Chandler, H.W., A three dimensional computational model of the mechanical response of a dual-phase ceramic. Computational Materials Science, 2007. 39: p. 862-870.

[14] Li, S. and Ghosh, S., Extended Voronoi cell finite element model for multiple cohesive crack propagation in brittle materials. Int. J. Num. Meth. Engg, 2005. 65(7): p. 1028 1067.

[15] Tomar, V. and Zhou, M., Deterministic and stochastic analyses of dynamic fracture in two-phase ceramic microstructures with random material properties. Eng. Fract. Mech., 2005. 72: p. 1920-1941.

[16] Pandolfi, A., Yu, C., Corigliano, A., and Ortiz, M., Modeling dynamic fracture in transversely isotropic composites: a cohesive approach. 2000, California Institute of Technology.

[17] Xu, X.P. and Needleman, A., Numerical simulations of fast crack growth in brittle solids. J. Mech. Phys. Solids, 1994. 42: p. 1397-1434.

[18] Tomar, V., Zhai, J., and Zhou, M., Bounds for element size in a variable stiffness cohesive finite element model. Int. J. Num. Meth. Engg., 2004. 61: p. 1894-1920.

[19] Klopp, R.W. and Shockey, D.A., The strength behavior of granulated silicon carbide at high strain 
rates and confining pressure. J. Appl. Phys., 1991. 70(12): p. 7318-7326.

[20] Holmquist, T.J. and Johnson, G.R., Response of silicon carbide to high velocity impact. J. Appl. Phys., 2002. 91(9): p. 5858-5866.

[21] Walker, J., Analytically modeling hypervelocity penetration of thick ceramic targets. int. J. Impact Engg., 2003. 29(1-10): p. 747-755.

[22] Loubens, A., Rivero, C., Boivin, P., Charlet, B., Fortunier, R., and Thomas, O. Investigation of local stress fields: Finite element modeling and high-resolution X-ray diffraction. 2005.

[23] Ortiz, M. and Pandolfi, A., Finite deformation irreversible cohesive elements for three dimensional crack-propagation analysis. Int. J. Num. Meth. Engg., 1999. 44(9): p. 12671282.

[24] Shet, C. and Chandra, N., Analysis of energy balance when using cohesive zone models to simulate fracture processes. J. Engg. Mat. Tech., 2002. 124: p. 440-450.

[25] Minnaar, K., Experimental and numerical analysis of damage in laminate composites under low velocity impact loading. 2002, PhD Thesis, Georgia Institute of Technology, Atlanta, GA.

[26] Tvergaard, V., Cohesive zone representations of failure between elastic or rigid and ductile solids. Eng. Fract. Mech., 2003. 70: p. 1859-1868.

[27] Camacho, G.T. and Ortiz, M., Computational modeling of impact damage in brittle materials. International Journal of Solids and Structures, 1996. 33: p. 2899-2938.

[28] Zhai, J., Tomar, V., and Zhou, M., Micromechanical modeling of dynamic fracture using the cohesive finite element method. J. Engg. Mat. Tech., 2004. 126: p. 179-191.

[29] Sorensen, B.F. and Jacobsen, T.K., Determination of cohesive laws by the J integral approach. Engineering Fracture Mechanics, 2003. 70: p. 1841-1858.

[30] Cornec, A., Scheider, I., and Schwalbe, K.-H., On the practical application of the cohesive zone model. Engineering Fracture Mechanics, 2003. 70: p. 1963-1987.

[31] Espinosa, H.D., Dwivedi, S., and Lu, H.-C., Modeling impact induced delamination of woven fiber reinforced composites with contact/cohesive laws. Computer Methods in Applied Mechanics and Engineering, 2000. 183: p. 259-290.

[32] Niihara, K., Suganuma, K., Nakahira, A., and Izaki, K., Interfaces in Si $i_{3} \mathrm{~N}_{4}-\mathrm{SiC}$ nanocomposites. J. Mater. Sci. Letters, 1990. 9(1990): p. 598-599.

[33] Schwetz, K.A., Kempf, T., Saldsleder, D., and Telle, R., Toughness and hardness of LPSSiC and LPS-SiC based composites. Ceramic engineering and science proceedings, 2004. 25(3): p. 579-588.

[34] Messier, D.R. and Croft, W.J., Silicon Nitride. 1982, Army Research LaboratoryAMMRC TR 82-42.

[35] Liu, X.-J., Huang, Z.-Y., Pu, X.-P., Subn, X.-W., and Huang, L.-P., Influence of planetary high-energy ball milling on microstructure and mechanical properties of silicon nitride ceramics J. Am. Ceram. Soc., 2005. 88(5): p. 1323-1326.

[36] Blugan, G., Hadad, y.M., Janczak-Rusch, J., Kuebler, J., and Graulez, T., Fractography, mechanical properties, and microstructure of commercial silicon nitride-Titanium nitride composites. J. Am. Ceram. Soc., 2005. 88(4): p. 926-933.

[37] Belytschko, T., Chiapetta, R.L., and Bartel, H.D., Efficient large scale non-linear transient analysis by finite elements. Int. J. Num. Meth. Engg., 1976. 10: p. 579-596. 
[38] Zhai, J. and Zhou, M., Finite element analysis of micromechanical failure modes in heterogeneous brittle solids. International Journal of Fracture, special issue on Failure Mode Transition in Solids, 2000. 101: p. 161-180.

[39] Ravi-Chandar, K. and Knauss, W.G., An experimental investigation into dynamic fracture IV. On the interaction of stress waves with propagating cracks. Int. J. Fracture, 1984. 26: p. 192-203.

[40] Wan, J., Duan, R.-G., Gasch, M.J., and Mukherjee, A.K., Highly creep-resistant Silicon Nitride/Silicon Carbide nano-nano composites. J. Am. Ceram. Soc., 2006. 89(1): p. 274280.

[41] Perez-Regueiro, J., Pastor, J.Y., Llorca, J., Elices, M., Miranzo, P., and Moya, J.S., Revisiting the mechanical behavior of aluminum/silicon carbide nanocomposites Acta Mater., 1998. 46(15): p. 5399-5411.

[42] Choi, H.-J., Cho, K.-S., and Lee, J.-G., R-curve behavior of Silicon-nitride-Titanium Nitride composites. J. Am. Ceram. Soc., 1997. 10(10): p. 2681-2684.

[43] Tomar, V., Analyses of the role of the second phase SiC particles in microstructure dependent fracture resistance variation of $\mathrm{SiC}_{-} \mathrm{Si}_{3} \mathrm{~N}_{4}$ nanocomposites. Modelling Simul. Mater. Sci. Eng. , 2008. 16: p. 035001.

[44] Tomar, V., Analyses of the role of grain boundaries in mesoscale dynamic fracture resistance of $\mathrm{SiC}_{-} \mathrm{Si}_{3} \mathrm{~N}_{4}$ intergranular nanocomposites. Eng. Fract. Mech., 2008. 75: p. 4501-4512.

[45] Farkas, D., Willemann, M., and Hyde, B., Atomistic mechanisms of fatigue in nanocrystalline metals. Phys. Rev. Lett., 2005. 94: p. 165502.

[46] Yamakov, V., Wolf, D., Phillpot, S.R., and Gleiter, H., Deformation twinning in nanocrystalline Al by moleculardynamics simulation. Acta Materialia, 2002. 50: p. 50055020.

[47] Liao, X.Z., Zhou, F., Lavernia, E.J., He, D.W., and Zhua, Y.T., Deformation twins in nanocrystalline Al. Appl. Phys. Lett., 2003. 83(24): p. 5062-5064.

[48] Liao, X.Z., Zhou, F., Lavernia, E.J., Srinivasan, S.G., Baskes, M.I., He, D.W., and Zhu, Y.T., Appl. Phys. Lett., 2003. 83: p. 632.

[49] Abraham, F.F., How fast can cracks move? A research adventure in materials failure using millions of atoms and big computers. Advances in Physics, 2003. 52(8): p. 727-790.

[50] Kadau, K., Germann, T.C., Lomdahl, P.S., and Holian, B.L., Microscopic view of structural phase transitions induced by shock waves. Science, 2002. 296: p. 1681.

[51] Dionald, W.A.R.D., Curtin, W.A., and Yue, Q., Mechanical behavior of aluminumsilicon nanocomposites : A molecular dynamics study. Acta Materialia, 2006. 54(17): p. 4441-4451.

[52] Song, M. and Chen, L., Molecular dynamics simulation of the fracture in polymerexfoliated layered silicate nanocomposites. Macromolecular Theory and Simulations, 2006. 15(3): p. 238-245.

[53] Tomar, V. and Zhou, M., Analyses of tensile deformation of nanocrystalline $\alpha$ $\mathrm{Fe} 2 \mathrm{O} 3+f c c-A l$ composites using classical molecular dynamics. Journal of the Mechanics and Physics of Solids, 2007. 55: p. 1053-1085.

[54] Zeng, Q.H., Yu, A.B., and Lu, G.Q., Molecular dynamics simulations of organoclays and polymer nanocomposites. Int. J. Nanotechnology, 2008. 5(2-3): p. 277-290. 
[55] Zeng, Q.H., Yu, A.B., Lu, G.Q., and Standish, R.K., Molecular dynamics simulation of organic-inorganic nanocomposites: Layering behavior and interlayer structure of organoclays. Chem. Mater., 2003. 15: p. 4732-4738.

[56] Tsuruta, K., Totsuji, H., and Totsuji, C., Neck formation processes of nanocrystalline silicon carbide: A tight-binding molecular dynamics study. Philosophical Magazine Letters, 2001. 81(5): p. 357.

[57] Tsuruta, K., Totsuji, H., and Totsuji, C., Parallel tight-binding molecular dynamics for high-temperature neck formation processes of nanocrystalline silicon carbide. Materials Transactions, 2001. 42(11): p. 2261.

[58] Mirgorodsky, A.P., Baraton, M.I., and Quintard, P., Lattice dynamics and prediction of pressure-induced incommensurate instability of a $\beta-S_{3} N_{4}$ lattice with a simple mechanical model. Phys. Rev. B, 1993. 48(18): p. 13326-13332.

[59] Lidorikis, E., Bachlechner, M.E., Kalia, R.K., Nakano, A., Vashishta, P., and Voyiadjis, G.Z., Coupling length scales for multiscale atomistic-continuum simulations: Atomistically induced stress distributions in $\mathrm{Si} \mathrm{Si}_{3} \mathrm{~N}_{4}$ nanopixels. Phys. Rev. Lett., 2001. 87(8): p. 086104.

[60] Tomar, V. and Zhou, M., Tension-compression strength asymmetry of nanocrystalline $\alpha$ $\mathrm{Fe}_{2} \mathrm{O}_{3}+f c c$-Al ceramic-metal composites. Appl. Phys. Lett., 2006. 88: p. 233107 (1-3).

[61] Wendel, J.A. and Goddard, W.A., The Hessian biased force field for silicon nitride ceramics: Predictions of thermodynamic and mechanical properties for $\alpha$ - and $\beta-\mathrm{Si}_{3} \mathrm{~N}_{4}$. J. Chem Phys., 1992. 97(7): p. 5048-5062.

[62] Mota, F.d.B., Justo, J.F., and Fazzio, A., Hydrogen role on the properties of amorphous silicon nitride. J. Appl. Phys., 1999. 86(4): p. 1843-1847.

[63] Kroll, P.M., Computer simulations and X-ray absorption near edge structure of silicon nitride and silicon carbonitride. 1996, Ph.D. Thesis, Technische Hochschule Darmstadt, Darmstadt, Germany.

[64] Ching, W.-Y., Xu, Y.-N., Gale, J.D., and Ruehle, M., Ab-Initio total energy calculation of $\alpha$-and $\beta$-silicon nitride and the derivation of effective pair potentials with application to lattice dynamics. J. Am. Ceram. Soc., 1998. 81(12): p. 3189-3196.

[65] Morkoc, H., Strite, S., Gao, G.B., Lin, M.E., Sverdlov, B., and Burns, M., Large-bandgap SIC, Ill-V nitride, and II-VI ZnSe-based semiconductor device technologies J. Appl. Phys., 1994. 76(3): p. 1363-1398.

[66] Tersoff, J., Empirical interatomic potential for silicon with improved elastic properties. Phys. Rev. B, 1988. 38: p. 9902-9905.

[67] Tersoff, J., Modeling solid-state chemistry: Interatomic potentials for multicomponent systems. Phys. Rev. B (Rapid Comm.), 1989. 39(8): p. 5566-5568.

[68] Tersoff, J., Carbon defects and defect reactions in silicon. Phys. Rev. Lett., 1990. 64: p. 1757-1760.

[69] Hanchen Huang, Nasr M Ghoniem, Jimmy K Wong, and Baskes, M.I., Molecular dynamics determination of defect energetics in $\beta$-SiC using three representative empirical potentials. Model. Sim. Mater. Sci. Engg., 1995. 1995(3): p. 615-627.

[70] Noreyan, A., Amar, J.G., and Marinescu, I., Molecualr dynamics simulations of nanoindentation of $\beta$-SiC with diamond indentor. Mater. Sci. Engg. B, 2005. 117: p. 235240.

[71] Matsunaga, K., Fisher, C., and Matsubara, H., Tersoff potential parameters for simulating cubic boron carbonitrides. Jpn. J. Appl. Phys., 2000. 39: p. L48-L51. 
[72] Tomar, V., Atomistic modeling of the $\mathrm{Al}+\mathrm{Fe}_{2} \mathrm{O}_{3}$ material system using classical molecular dynamics, in Mechanical Engineering. 2005, Georgia Institute of Technology: Atlanta. p. 295.

[73] Smith, W., Yong, C.W., and Rodger, P.M., DL_POLY: Application to molecular simulation. Mol. Sim., 2002. 28(5): p. 385-471.

[74] Schiøtz, J., Di Tolla, F.D., and Jacobsen, K.W., Softening of nanocrystalline metals at very small grain sizes. Nature, 1998. 391: p. 561-563.

[75] Van Swygenhoven, H. and Caro, A., Plastic behavior of nanophase Ni: A molecular dynamics computer simulation. Appl. Phys. Lett., 1997. 71(12): p. 1652-1654.

[76] Spearot, D.E., Jacob, K.I., and McDowell, D.L., Nucleation of dislocations from [001] bicrystal interfaces in aluminum. Acta Materialia, 2005. 53: p. 3579-3589.

[77] Schiøtz, J., Vegge, T., Di Tolla, F.D., and Jacobsen, K.W., Atomic-scale simulations of the mechanical deformation of nanocrystalline metals. Phys. Rev. B, 1999. 60: p. 1197111983.

[78] Melchionna, S., Ciccotti, G., and Holian, B.L., Hoover NPT dynamics for systems varying in shape and size. Mol. Phys., 1993. 78(3): p. 533-544.

[79] Zhou, M., A new look at the atomic level virial stress- On continuum-molecular system equivalence. Proc. Royal Soc. London A, 2003. 459: p. 2347-2392.

[80] Haftka, R.T., Gürdal, Z., and Kamat, M.P., Elements of Structural Optimization. $2^{\text {nd }}$ ed. ed. 1990, Waterloo,: Kluwer Academic Publishers,. 341-376.

[81] Bruyneel, M., A General and Efective Approach for the Optimal Design of Fiber Reinforced Composite Structures. Composites Science and Technology, 2005,. Vol. 66,: p. pp. 1303-1314.

[82] Ikeda, Y., A new method of alloy design using a genetic algorithm and molecular dynamics simulation and its application to nickel-based superalloys. Materials Transactions Jim, 1997. 38(9): p. 771-779.

[83] Kulkarni, A.J., Krishnamurthy, K., Deshmukh, S.P., and Mishra, R.S., Microstructural optimization of alloys using a genetic algorithm. Materials Science And Engineering AStructural Materials Properties Microstructure And Processing, 2004. 372(1-2): p. 213220.

[84] Sadagopan, D. and Pitchumani, R., Application of genetic algorithms to optimal tailoring of composite materials. Composites Science And Technology, 1998. 58(3-4): p. 571-589.

[85] Olson, G.B., Computational Design of Hierarchically Structured Materials. Science, 1997. 277(5330): p. 1237-1412.

[86] Ashby, M.F., Mulit-Objective Optimization in Material Design and Selection. Acta Materialia, 2000. 48(1): p. 359-369.

[87] Ashby, M.F. and Bréchet, P., Designing Hybrid Materials. Acta Materialia, 2003. 51(19): p. 5801-5821.

[88] Seepersad, C.C., Allen, J.K., McDowell, D.L., and Mistree, F., Robust Design of Cellular Materials With Topological and Dimensional Imperfections. Journal of Mechanical Design, 2006. 128(6): p. 1285-1297.

[89] McVeigh, C., Vernerey, F., Liu, W.K., and Cate, L.B., Multiresolution Analysis for Material Design. Computer Methods in Applied Mechanics and Engineering, 2006. 195(37-40): p. 5053-5076. 
[90] Pelegri, A.A. and Tekkam, A., Optimization of Laminates' Fracture Toughness Using Design of Experiments and Response Surface. Journal of Composite Materials, 2003. 37(7): p. 579-596.

[91] Tomar, V. and Zhou, M., Deterministic and Stochastic Analyses of Dynamic Fracture in Two-Phase Ceramic Microstructures with Random Material Properties. Eng. Fract. Mech., 2005. 72(12): p. 1920-1941.

[92] Mejía-Rodríguez, G., Renaud, J.E., and Tomar, V., A variable fidelity model management framework for designing multiphase materials. Journal Of Mechanical Design, 2008. 130(9): p. 091702 (1-13)

[93] Mejía-Rodríguez, G., Renaud, J.E., and Tomar, V., Multiobjective Ceramic Matrix Composite Material Design Using the Variable Fidelity Model Management Optimization Framework. Engineering Optimization. 2010. In Press.

[94] Lei, S., Shin, Y.C., and Incropera, F., Deformation Mechanisms and Constitutive Modeling for Silicon Nitride Undergoing Laser-Assisted Machining. International Journal of Machine Tools \& Manufacture, 2000. 40(15): p. 2213-2233.

[95] Wiederhorn, S.M., Hockey, B.J., and French, J.D., Mechanisms of Deformation of Silicon Nitride and Silicon Carbide at High Temperatures. Journal of the European Ceramic Society, 1999. 19(12): p. 2273-2284.

[96] Rouxel, T. and Besson, J.L., Viscoplastic Forming of $\mathrm{Si}_{3} \mathrm{~N}_{4}$-Based Ceramic and GlassMatrix Particulate Composites. Journal of the European Ceramic Society, 1997. 17(1): p. 1963-1966.

[97] Krause, R.F., Luecke, W.E., French, J.D., Hockey, B.J., and Wiederhorn, S.M., Tensile Creep and Rupture of Silicon Nitride. Journal of The American Ceramic Society, 1999. 82(5): p. 1233-1241.

[98] Wiederhorn, S.M. and Luecke, W.E., Creep of Silicon Nitride, in In Computer Aided Design of High Temperature Materials, ed. A. Pechenik, R. K. Kalia and P. Vashishta, . 1998, Oxford University Press,: Oxford.

[99] Kusunose, T., Sung, R.J., Sekino, T., Sakaguchi, S., and Niihara, K., High-temperature properties of a silicon nitride/boron nitride nanocomposite. Journal Of Materials Research, 2004. 19(5): p. 1432-1438.

[100] Park, H., Kim, H.E., and Niihara, K., Microstructure and high-temperature strength of Si3N4-SiC nanocomposite. Journal Of The European Ceramic Society, 1998. 18(7): p. 907-914.

[101] Lane, J.E., Carter, C.H., and Davis, R.F., Kinetics and Mechanisms of High-Temperature Creep in Silicon-Carbide: III. Sintered Alpha-Silicon Carbide. Journal of the American Ceramic Society, 1988. 71(4): p. 281-295.

[102] Munro, R.G., Material properties of a sintered alpha-SiC. Journal Of Physical And Chemical Reference Data, 1997. 26(5): p. 1195-1203.

[103] Tomar, V. and Samvedi, V., Atomistic Simulations-Based Understanding of the Mechanism behind the Role of Second-Phase SiC Particles in Fracture Resistance of SiC-Si3N4 Nanocomposites. International Journal For Multiscale Computational Engineering, 2009. 7(4): p. 277-294.

[104] Tomar, V., Analyses of the role of the second phase SiC particles in microstructure dependent fracture resistance variation of SiC-Si3N4 nanocomposites. Modelling And Simulation In Materials Science And Engineering, 2008. 16(3). 
[105] Tomar, V., Analyses of the role of grain boundaries in mesoscale dynamic fracture resistance of $\mathrm{SiC}_{-} \mathrm{Si}_{3} \mathrm{~N}_{4}$ intergranular nanocomposites. Engineering Fracture Mechanics, 2008. 75(15): p. 4501-4512.

[106] Johnson, W.A. and Mehl, R.F., Reaction Kinetics in Processes of Nucleation and Growth, Trans. Am. Inst. Min. Metall., 1939. 135: p. 416-458.

[107] Frost, H.J. and Thompson, C.V., The Effect Of Nucleation Conditions On The Topology And Geometry Of Two-Dimensional Grain Structures. Acta Metallurgica, 1987. 35(2): p. 529-540.

[108] Mahin, K.W., Hanson, K., and Morris, J.W., Comparative-Analysis Of The Cellular And Johnson-Mehl Microstructures Through Computer-Simulation. Acta Metallurgica, 1980. 28(4): p. 443-453.

[109] Meijering, J.L., Interface Area, Edge Length, and Number of Vertices in Crystal Aggregates with Random Nucleation. Philips Res. Rep.,, 1953. 8: p. 270-290.

[110] Powers, L.M., Mechanical Behavior of Ceramics at High Temperatures: Constitutive Modeling and Numerical Implementation, in Civil Engineering. 2006, Case Western Reserve University: Cleveland.

[111] Choi, J., Kim, H., and Lee, J.K., Lattice Based Microstructure Evolution Model for Monte Carlo Finite Element Analysis of Polycrystalline Materials. Materials Processing and Design: Modeling, Simulation and Applications. 2004, Columbus, Ohio American Institute of Physics: Numiform conference proceedings, edited by Ghosh et al. pp. 18241829.

[112] Weaire, D., Kermode, J.P., and Wejchert, J., On the distribution of cell areas in a Voronoi network. Philosophical Magazine B, 1986. 53(5): p. L101-L105.

[113] Mulheran, P., The scaling state in two-dimensional grain growth. Acta Metallurgica et Materialia, 1994. 42(11): p. 3589-3593.

[114] Phadke, M.S., Quality Engineering Using Robust Design. 1989, Englewood Cliffs, NJ: Prentice Hall.

[115] Su, J. and Renaud, J.E., Automatic differentiation in robust optimization. AIAAJournal, 1997. 35(6): p. 1072-1079.

[116] Chen, W., A robust concept exploration method for configuring complex systems, in Mechanical engineering. 1995, Georgia Institute of Technology.

[117] Gu, X., Renaud, J.E., Batill, S.M., Brach, R.M., and Budhiraja, A.S., Worst case propagated uncertainty of multidisciplinary systems in robust design optimization. Structural And Multidisciplinary Optimization, 2000. 20(3): p. 190-213.

[118] Chen, W., Wiecek, M.M., and Zhang, J., Quality utility - A compromise programming approach to robust design. Journal of Mechanical Design, 1999. 121(2): p. 179-187.

[119] Rodriguez, J.F., Perez, V.M., Padmanabhan, D., and Renaud, J.E., Sequential Approximate Optimization Using Variable Fidelity Response Surface Approximations. Structural and Multidisciplinary Optimization, 2001. 22(1): p. 24-34.

[120] Rodriguez, J.F., Renaud, J.E., and Watson, L.T., Trust Region Augmented Lagrangian Methods for Sequential Response Surface Approximation and Optimization. ASME Journal of Mechanical Design, 1998. 120(1): p. 58-66.

[121] Perez, V.M., Renaud, J.E., and Watson, L.T., An Interior-Point Sequential Approximate Optimization Methodology. Structural Optimization, 2004. 27(5): p. 360-370.

[122] Perez, V.M., Renaud, J.E., and Watson, L.T. Reduced Sampling for Construction of Quadratic Response Surface Approximations Using Adaptive Experimental Design. in 
Proceedings of the 43rd AIAA/ASME/ASCE/AHS/ASC Structures, Structural Dynamics, and Materials Conference. 2002. Denver, Colorado.

[123] Rasmussen, J., Nonlinear programming by cumulative approximation refinement. Structural Optimization, 1998. 15(1): p. 1-7.

[124] Li, H. and Ngan, A.H.W., Size effect of nanoindentation creep. J. Mater. Res., 2004. 19(2): p. 513-522.

[125] Chudoba, T. and Richter, F., Investigation of creep behavior under load during indentation experiments and its influence on hardness and modulus results. Surf. Coat. Technol., 2001. 148(2-3): p. 191-198.

[126] Li, W.B., Henshall, J.L., Hooper, R.M., and Easterling, K.E., The Mechanisms of Indentation Creep. Acta Metallurgica Et Materialia, 1991. 39(12): p. 3099-3110.

[127] Li, W.B., Henshall, J.L., Hooper, R.M., and Easterling, K.E., The mechanisms of indentation creep. Acta Metallurgia et. Materialia, 1991. 39(12): p. 3099-3110.

[128] Herring, C., Diffusional Viscosity of a Polycrystalline Solid. Journal of Applied Physics, 1950. 21(5): p. 437-445.

[129] Coble, R.L., A Model for Boundary Diffusion Controlled Creep in Polycrystalline Materials. Journal of Applied Physics, 1963. 34(6): p. 1679-1682.

[130] Lifshitz, I.M., On the Theory of Diffusion-Viscous Flow of Polycrystalline Bodies. Soviet Physics Jetp-Ussr, 1963. 17(4): p. 909-920.

[131] Weertman, J., Trans. ASM, 1968. 61: p. 681-694.

[132] Nix, W.D. and Ilschner, B. in Proc. 5th Int. Conf. on the Strength of Metals and Alloys (ICSMA 5). 1980. Oxford: Pergamon Press.

[133] Sherby, O.D. and Burke, P.M., Prog. Mater. Sci., 1968. 13: p. 323-390.

[134] Li, J.C.M., Buschow, K.H.J., Robert, W.C., Merton, C.F., Bernard, I., Edward, J.K., Subhash, M., and Patrick, V., Creep by Indentation, in Encyclopedia of Materials: Science and Technology. 2001, Elsevier: Oxford. p. 1765-1767.

[135] Li, H. and Ngan, A.H.W., Size Effects of Nanoindentation Creep. Journal of Materials Research, 2004. 19(2): p. 513-522.

[136] Feng, G. and Ngan, A.H.W., Creep and strain burst in indium and aluminium during nanoindentation. Scripta Materialia, 2001. 45(8): p. 971-976.

[137] Cao, Z.H., Li, P.Y., Lu, H.M., Huang, Y.L., Zhou, Y.C., and Meng, X.K., Indentation size effects on the creep behavior of nanocrystalline tetragonal Ta films. Scripta Materialia, 2009. 60(6): p. 415-418.

[138] Mayo, M.J. and Nix, W.D., A micro-indentation study of superplasticity in $\mathrm{Pb}, \mathrm{Sn}$, and Sn-38 wt\% Pb. Acta Metallurgica, 1988. 36(8): p. 2183-2192.

[139] Lucas, B. and Oliver, W., Indentation power-law creep of high-purity indium. Metallurgical and Materials Transactions A, 1999. 30(3): p. 601-610.

[140] Asif, S.A.S. and Pethica, J.B., Nanoindentation creep of single-crystal tungsten and gallium arsenide. 1997, Taylor \& Francis. p. 1105 - 1118.

[141] Zong, Z. and Soboyejo, W., Indentation size effects in face centered cubic single crystal thin films. Materials Science and Engineering: A, 2005. 404(1-2): p. 281-290.

[142] Durst, K., Goken, M., and Pharr, G.M., Indentation size effect in spherical and pyramidal indentations. Journal of Physics D: Applied Physics, 2008. 41(7): p. 074005.

[143] Durst, K., Backes, B., Franke, O., and Göken, M., Indentation size effect in metallic materials: Modeling strength from pop-in to macroscopic hardness using geometrically necessary dislocations. Acta Materialia, 2006. 54(9): p. 2547-2555. 
[144] Li, H. and Ngan, A.H.W., Indentation size effects on the strain rate sensitivity of nanocrystalline Ni-25at.\%Al thin films. Scripta Materialia, 2005. 52(9): p. 827-831.

[145] Jan, V., Dorcakova, F., Dusza, J., and Bartsch, M., Indentation creep of free-standing EB-PVD thermal barrier coatings. Journal of the European Ceramic Society, 2008. 28(1): p. 241-246.

[146] Chollon, G., Vallerot, J.M., Helary, D., and Jouannigot, S., Structural and textural changes of CVD-SiC to indentation, high temperature creep and irradiation. Journal of the European Ceramic Society, 2007. 27(2-3): p. 1503-1511.

[147] Janakiraman, N. and Aldinger, F., Yielding, strain hardening, and creep under nanoindentation of precursor-derived Si-C-N ceramics. J. Am. Ceram. Soc., 2010. 93(3): p. 821-829.

[148] Janakiraman, N. and Aldinger, F., Indentation analyses of elastic and plastic deformation of precursor derived Si-C-N ceramics. J. Eur. Ceram. Soc., 2010. 30(3): p. 775-785.

[149] Olbricht, J., Yawny, A., Young, M.L., and Eggeler, G., Mechanical and microstructural observations during compression creep of a short fiber reinforced AlMg metal matrix composite. Materials Science and Engineering: A, 2009. 510-511: p. 407-412.

[150] Khan, K.B., Kutty, T.R.G., and Surappa, M.K., Hot hardness and indentation creep study on Al-5\% Mg alloy matrix-B4C particle reinforced composites. Materials Science and Engineering: A, 2006. 427(1-2): p. 76-82.

[151] Torrey, J.D. and Bordia, R.K., Mechanical properties of polymer-derived ceramic composite coatings on steel. Journal of the European Ceramic Society, 2008. 28(1): p. 253-257.

[152] Torrey, J.D., Bordia, R.K., Henager Jr., C.H., Blum, Y., Shin, Y., and Samuels, W.D., Composite polymer derived ceramic system for oxidizing environments. Journal of Materials Science, 2006. 41: p. 4617-4622.

[153] Torrey, J.D. and Bordia, R.K., Mechanical properties of polymer-derived ceramic composite coatings on steel. J. Euro. Ceram. Soc., 2008. 28: p. 253-257.

[154] Torrey, J.D. and Bordia, R.K., Processing of polymer-derived ceramic composite coatings on steel. J. Am. Ceram. Soc., 2008. 91(1): p. 41-45.

[155] Torrey, J.D. and Bordia, R.K., Phase and microstructural evolution in polymer derived composite systems and coatings. Journal of Materials Research, 2007. 22(7): p. 19591966

[156] Guicciardi, S., Melandri, C., and Tullio Monteverde, F., Characterization of pop-in phenomena and indentation modulus in a polycrystalline ZrB2 ceramic. J. Eur. Ceram. Soc., 2010. 30(2010): p. 1027-1034.

[157] Oliver, W.C. and Pharr, G.M., Measurement of hardness and elastic modulus by instrumented indentation: Advances in understanding and refinements to methodology. $\mathrm{J}$. Mater. Res., 2004. 19(1): p. 3-20.

[158] Oliver, W.C. and Pharr, G.M., An improved technique for determining hardness and elastic modulus using load and displacement sensing indentation. J. Mater. Res., 1992. 7(6): p. 1564-1583.

[159] Bower, A.F., Fleck, N.A., Needleman, A., and Ogbonna, N., Indentation of a Power Law Creeping Solid. The Royal Society Proceedings: Mathematical and Physical Sciences, 1993. 441(1911): p. 97-124.

[160] Raman, V. and Berriche, R., An investigation of the creep processes in tin and aluminum using a depth-sensing indentation technique. J. Mater. Res., 1992. 7(3): p. 627-638. 
[161] Weihs, T.P. and Pethica, J.B. Monitoring time-dependent deformation in small volumes. in Materials Research Society Symposia Proceedings. 1992. Pittsburgh, PA.

[162] Lucas, B.N. and Oliver, W.C., Indentation power-law creep of high-purity indium. Metall. Mater. Trans., 1999. 30A: p. 601-610.

[163] Herbert, E.G., Pharr, G.M., Oliver, W.C., Lucas, B.N., and Hay, J.L., On the measurement of stress-strain curves by spherical indentation. Thin Solid Films, 2001. 398-399: p. 331-335.

[164] Tabor, D., Indentation hardness: Fifty years on-A personal view. Phil Mag. A, 1996. 74(5): p. 1207-1212.

[165] Cheng, Y.T. and Cheng, C.M., Scaling, dimensional analysis, and indentation measurements. Mater. Sci. Eng. R, 2004. 44(4-5): p. 91-149.

[166] Ma, Z.S., Long, S.G., Zhou, Y.C., and Pan, Y., Indentation scale dependence of tip-in creep behavior in Ni thin films. Scripta Materialia, 2008. 59(2): p. 195-198.

[167] Medved', A.I. and Bryukhanov, A.E., The variation of Young's modulus and the hardness with tempering of some quenched chromium steels Metal Science and Heat Treatment, 2004. 11(9): p. 706-708.

[168] Cheng, T. and Cheng, C.M., Scaling, dimensional analysis, and indentation measurements. Mater. Sci. Eng. R., 2004. 44(4-5): p. 91-149.

[169] Christian, J.W., The Theory of Transformations in Metals and Alloys. 1975, Pergamon Press: Oxford, UK. p. 81.

[170] Ashby, M.F. and Frost, H.J., The kinetics of plastic deformation above 0K, in constitutive Equations in Plasticity, A.S. Argon, Editor. 1975, MIT Press: Cambridge, MA. p. 119.

[171] Puthoff, J.B., Jakes, J.E., Cao, H., and Stone, D.S., Investigation of thermally activated deformation in amorphous PMMA and $\mathrm{Zr}$-Cu-Al bulk metallic glasses with broadband nanoindentation creep. Journal of Materials Research, 2009. 24(3): p. 1279-1290. 


\section{VII-Acknowledgements and List of Publications From Grant Support}

PIs acknowledge support from the university coal research program of the department of energy and the support of program managers: Dr. Patricia Rawls and Dr. Nicholas Anderson.

Number of PhD Students Fully and Partially Supported by Grant: 4

Number of PhDs awarded: 1

Number of International Journal Publications: 12

\section{Tangible Benefit to DoE besides Education and Journal Publications:}

A software based methodology to design microstructure of particle based ceramic nanocomposites has been developed. This methodology has been shown to predict changes in phase morphologies required for achieving optimal balance of conflicting properties such as minimal creep strain rate and high fracture strength at high temperatures. The methodology incorporates complex material models including atomistic approaches. The methodology will be useful to design materials for high temperature applications including those of interest to DoE while significantly reducing cost of expensive experiments.

\section{International Journal Publications (Accepted/Published/Submitted)}

1. Mejia-Rodriguez, G., Renaud, J.E., Kim, H., and Tomar V., 2010, Sequential approximate optimization based robust design of $\mathrm{Sic}_{-} \mathrm{Si}_{3} \mathrm{~N}_{4}$ nanocomposite microstructures, Submitted June 152010

1. Gan, M, and Tomar, V., 2010, Role of length scale and temperature in indentation induced creep behavior of polymer derived Si-C-O ceramics, Accepted to Appear in Materials Science and Engineering-A, August 06, 2010, Manuscript ID: MSEA-D-100176, DOI: 10.1016/j.msea.2010.08.016

2. Mejia-Rodriguez, G., Renaud, J.E., and Tomar V., 2010, Multiobjective composite material design using the variable fidelity model management optimization framework, in press in Engineering Optimization

3. Tomar, V. and Gan, M., 2010, Temperature dependent nanomechanics of Si-C-N nanocomposites with an account of particle clustering and grain boundaries, in press in the Int. J. Hydrogen Energy, doi:10.1016/j.ijhydene.2010.03.070

4. Tomar, V., Gan, M., and Kim, H., 2010, Effect of temperature and morphology on mechanical strength of Si-C-O and Si-C-N nanocomposites, the Journal of European Ceramic Society, Volume 30, 2223-2237

5. Tomar, V., Samvedi, V., and Kim, H., 2010, Atomistic understanding of the particle clustering and particle size effect on the room temperature strength of SiC-Si3N4 nanocomposites to Appear in Int. J. Multiscale Comp. Engg. special issue on Advances In Computational Materials Science, volume 8 issue 4, 2010

6. Tomar, V., and Samvedi, V, 2009, Atomistic simulations based understanding of the mechanism behind the role of second phase $\mathrm{SiC}$ particles in fracture resistance of SiCSi3N4 nanocomposites, Vol 7, issue 4, 277-294 pages, International Journal of Multiscale Computational Engineering 
7. Samvedi, V, and Tomar, V., 2008, Role of heat flow direction, monolayer film thickness, and periodicity in controlling thermal conductivity of a Si-Ge superlattice system, Journal of Applied Physics (featured in Virtual Journal of Nanoscale Science and Technology, volume 19, issue 4), vol 105, 013541

8. Tomar, V., and Samvedi, V, 2009, Atomistic simulations based understanding of the mechanism behind the role of second phase $\mathrm{SiC}$ particles in fracture resistance of $\mathrm{SiC}$ Si3N4 nanocomposites, Vol 7, issue 4, 277-294 pages, International Journal of Multiscale Computational Engineering

9. Mejia-Rodriguez, G., Renaud, J.E., and Tomar V., 2008, A variable fidelity model management framework for designing multiphase materials, ASME J. Mech. Design, vol. $130,091702-1$

10. Tomar, V., 2008, Analyses of the role of the second phase $\mathrm{SiC}$ particles in microstructure dependent fracture resistance variation of $\mathrm{SiC}_{-} \mathrm{Si}_{3} \mathrm{~N}_{4}$ nanocomposites, Modelling Simul. Mater. Sci. Eng. 16 (2008) 035001.

11. Tomar, V., 2007, Nanomechanical analyses of nanocrystalline Ni using accelerated molecular timesteps, Physica Status Solidi-a, vol. 204 (10), pp. 3340-3348.

12. Tomar, V., 2007, Accelerating the molecular timesteps for nanomechanical simulations: The hybrid Monte Carlo Method, Journal of Applied Physics, Vol. 101, pp 103512 (1-9) 